M.Sc. Thesis - J. El-Sayes; McMaster University - Kinesiology

BIOLOGICAL SEX, OVARIAN HORMONES, AND EXERCISE-INDUCED NEUROPLASTICITY 
M.Sc. Thesis - J. El-Sayes; McMaster University - Kinesiology

\title{
THE EFFECTS OF BIOLOGICAL SEX AND OVARIAN HORMONES ON EXERCISE- INDUCED NEUROPLASTICITY
}

\author{
By JENIN EL-SAYES, B. Sc. (HONOURS) INTEGRATED SCIENCE
}

A Thesis Submitted to the School of Graduate Studies in Partial Fulfilment of the Requirements for the Degree of Master of Science

McMaster University @ Copyright by Jenin El-Sayes, August 2018 
M.Sc. Thesis - J. El-Sayes; McMaster University - Kinesiology

McMaster University MASTER OF SCIENCE (2018) Hamilton, Ontario (Kinesiology)

TITLE: The Effects of Biological Sex and Ovarian Hormones on Exercise-Induced Neuroplasticity

AUTHOR: Jenin El-Sayes, B. Sc. Integrated Science Honours (McMaster University)

SUPERVISOR: Dr. Aimee J. Nelson

NUMBER OF PAGES: xi, 80 


\title{
M.Sc. Thesis - J. El-Sayes; McMaster University - Kinesiology
}

\begin{abstract}
Acute aerobic exercise alters neurotrophic and growth factor concentrations, increases neural activity and improves cognitive and motor function. Additionally, acute exercise increases excitation and reduces inhibition in the motor cortex as assessed via transcranial magnetic stimulation (TMS). The propensity for exercise-induced neuroplasticity may be greater in females, as females show greater neuroplasticity induction following non-invasive brain stimulation (NIBS) compared to males. In females, NIBS yields greater neuroplasticity during ovulation, when estradiol levels are higher, compared to menstruation, suggesting that ovarian hormones may also impact exercise-induced neuroplasticity. To date, it is unknown whether biological sex and ovarian hormones impact neuroplasticity induced by acute aerobic exercise. The present thesis investigated the effects of biological sex and ovarian hormones on the magnitude and direction of neuroplasticity induced by acute aerobic exercise. Fourteen females and fourteen age- and fitnessmatched males participated in two sessions in which dependent measures were acquired before and following a single bout of aerobic exercise. Females were tested in the follicular ( day 7$)$ and luteal ( day 21) phases of the menstrual cycle and males were also tested on two occasions separated by $\sim 14$ days. The exercise intervention consisted of 5 minutes of warm up, 20 minutes of moderate intensity continuous cycling at 65-70\% maximal heart rate, and 5 minutes cool down. TMS was used to obtain motor evoked potential (MEP) recruitment curves and short interval intracortical inhibition (SICI) from the right first dorsal interosseous muscle. Blood measures of estradiol, progesterone, testosterone, and brain-derived neurotrophic factor were assessed before exercise on both testing sessions. Results indicated that only females showed increases in MEP recruitment curve following exercise, and this occurred regardless of menstrual cycle phase. SICI did not change following exercise for males or females on either testing session. The estrogen to
\end{abstract}


M.Sc. Thesis - J. El-Sayes; McMaster University - Kinesiology

progesterone ratio in females was greater in the follicular phase compared to the luteal phase. There was a trend towards greater serum BDNF levels in females in the luteal phase compared to the follicular phase. Sex hormones and BDNF levels did not vary across sessions in males. These findings indicate biological sex, but not ovarian hormones, is an important factor to consider when inducing neuroplasticity using acute exercise. 
M.Sc. Thesis - J. El-Sayes; McMaster University - Kinesiology

\section{Acknowledgements}

First and foremost, I would like to thank my supervisor Dr. Aimee Nelson for your continuous guidance and support. You have helped me overcome barriers and achieve my goals. The resilience and commitment you have helped instill in me has guided me through my Masters and will continue to do so later in life.

I would also like to thank my committee members Dr. Martin Gibala, Dr. Margaret Fahnestock, and Dr. Steve Bray for your advice. Your contributions have greatly improved the quality of my research.

Thank you to the members of The Neurophysiology and Imaging Lab, The High Performance Lab, and Dr. Fahnestock's Lab for your constant support in data collections, analyses, and conceptualization. Special thanks to Claudia Turco, Lauren Skelly, Chiara Nicolini, and Bernadeta Michalski for your devotion to the work and your continuous efforts to make it a success.

Last, thank you to my family and friends who have been a strong support system during this experience. 


\section{Table of Contents}

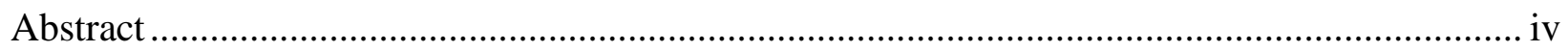

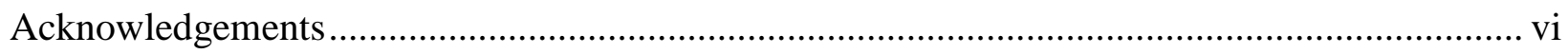

List of Tables and Figures........................................................................................... ix

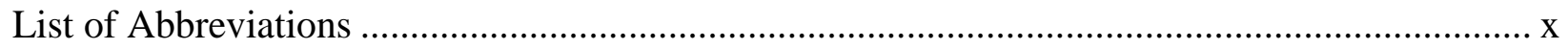

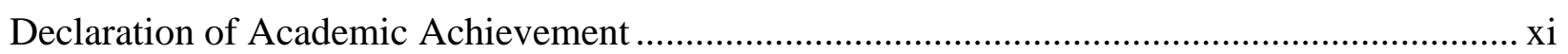

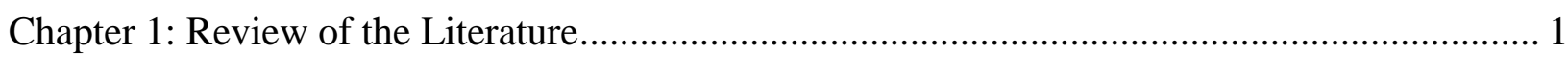

Exercise-Induced Neuroplasticity in Humans ................................................................. 2

Neurotrophic and Growth Factors ................................................................................... 2

Functional Connectivity and Cerebral Blood Flow …………............................................ 5

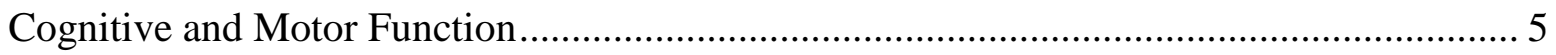



Transcranial Magnetic Stimulation: Assessing Neuroplasticity ............................................... 6

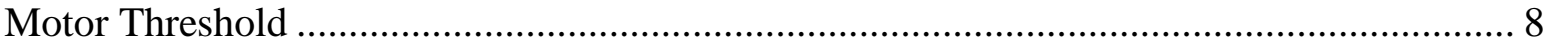

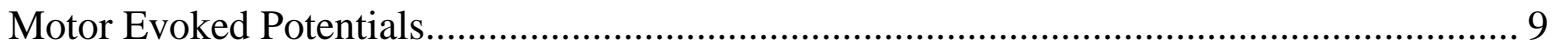

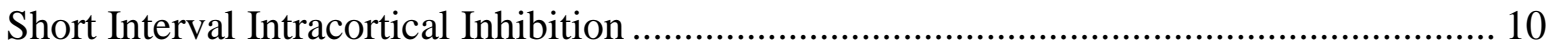

TMS to Measure Exercise-Induced Neuroplasticity ........................................................... 11

Factors Impacting Exercise-Induced Neuroplasticity ........................................................ 12

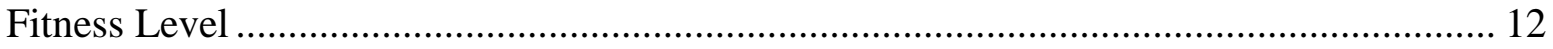

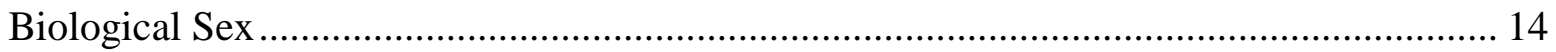

Menstrual Cycle and Ovarian Hormones...................................................................... 15

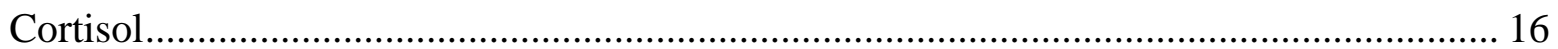

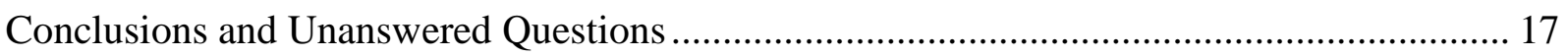

Chapter 2: Goals of Thesis and Significance ........................................................................... 18

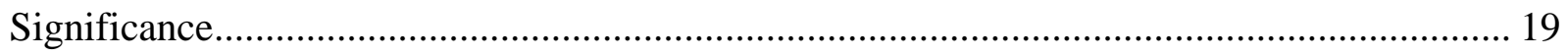

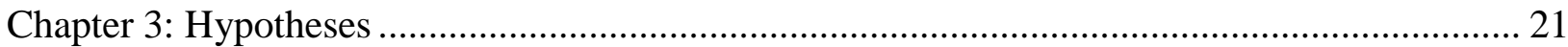

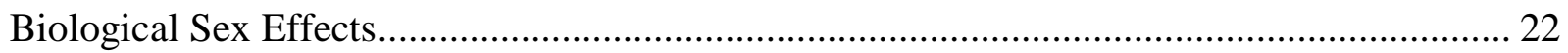

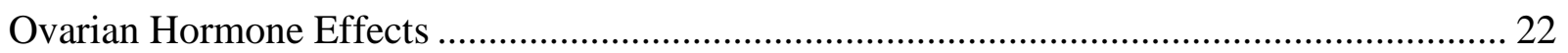

Chapter 4: Experiment - The Effects of Biological Sex and Ovarian Hormones on Exercise-

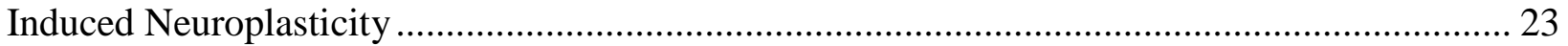

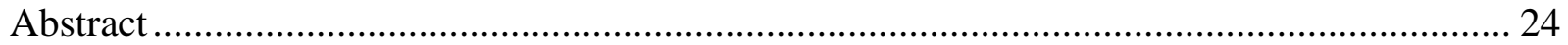

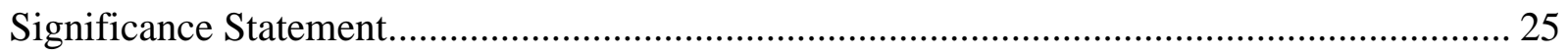




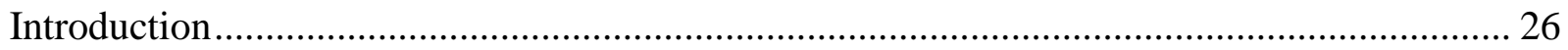

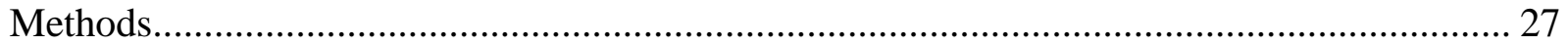

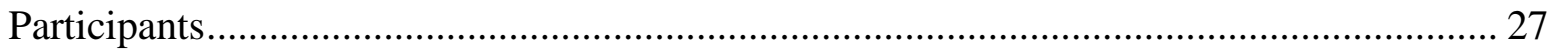

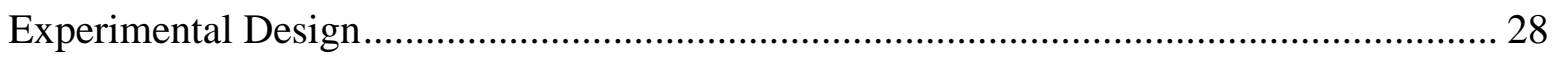

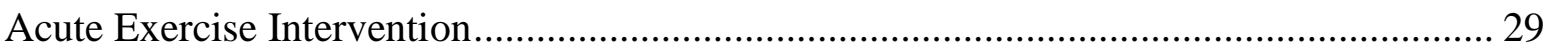



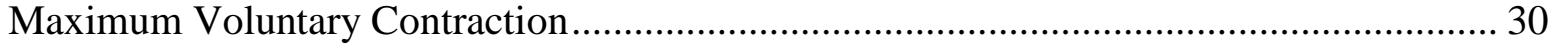

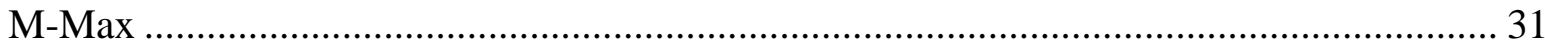

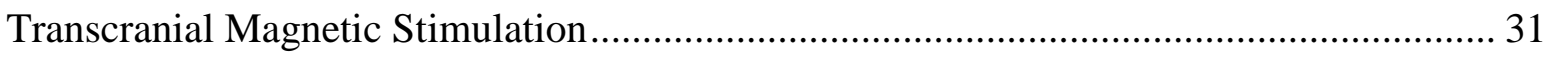

Hormone, Cortisol, and BDNF Analyses ............................................................... 32

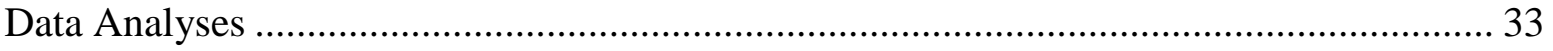

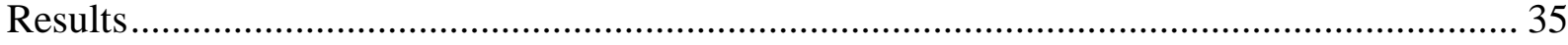

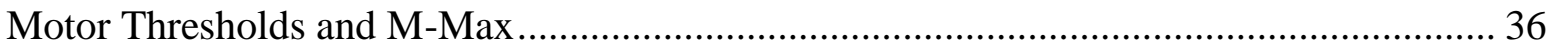



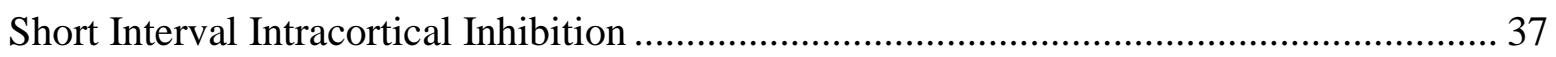

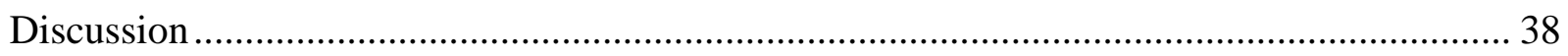

Increases in Corticospinal Excitability in Females ...................................................... 38

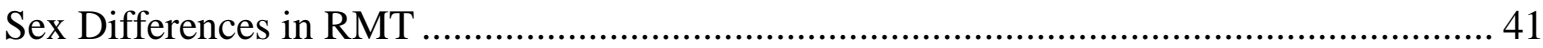

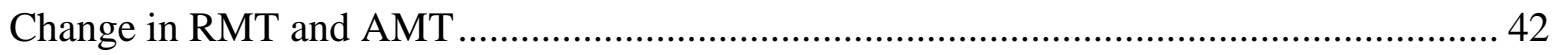



Limitations \& Future Considerations................................................................... 43

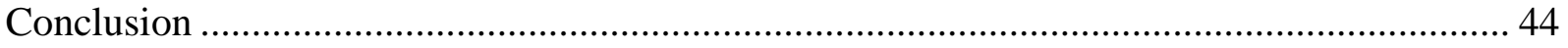

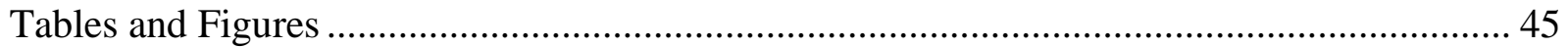

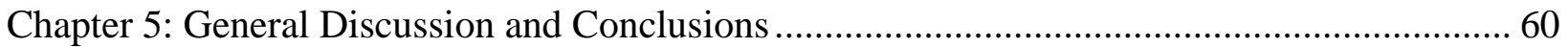

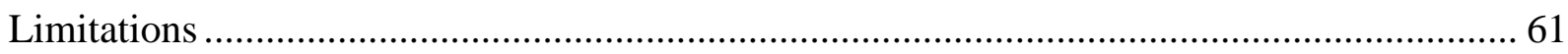

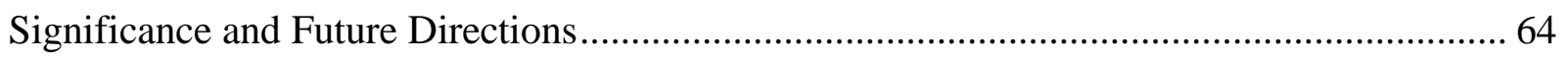

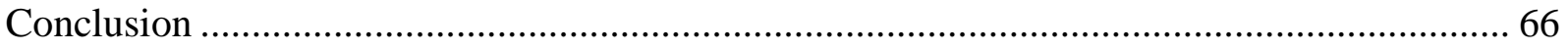

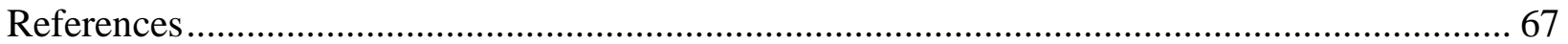


M.Sc. Thesis - J. El-Sayes; McMaster University - Kinesiology

\section{List of Tables and Figures}

\section{Literature Review}

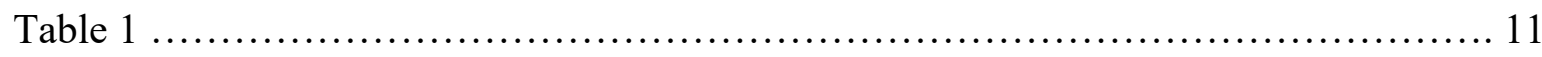

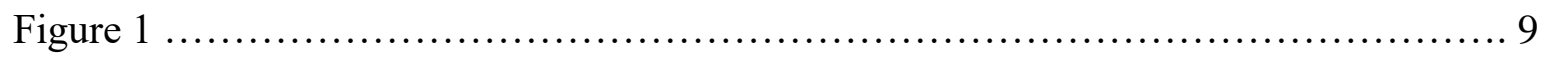

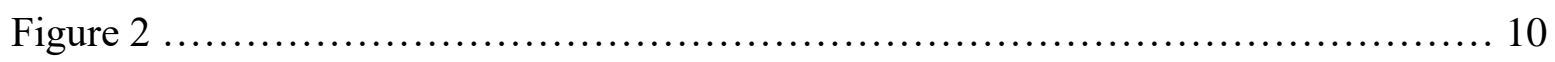



Experiment

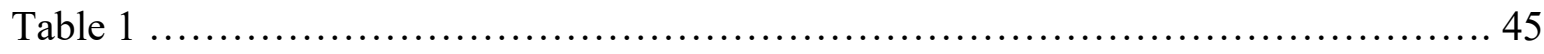

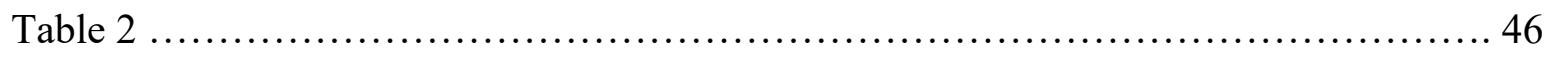

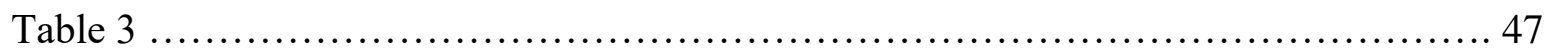

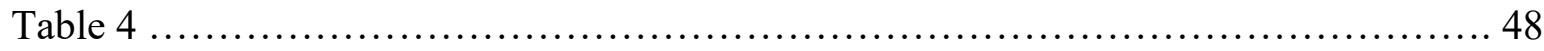

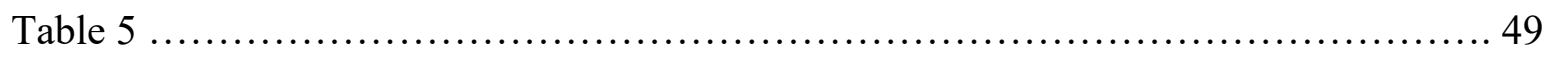

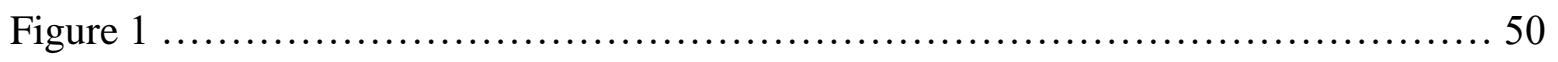

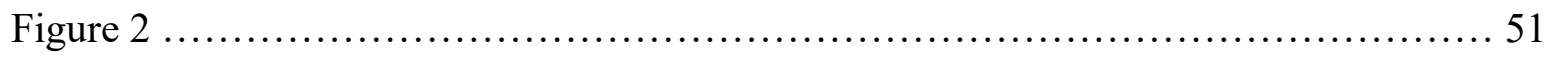

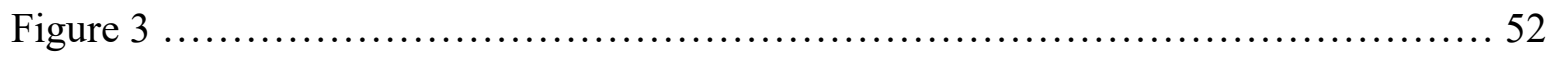

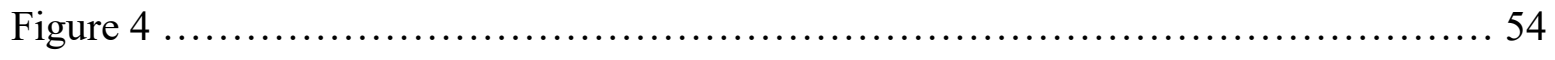

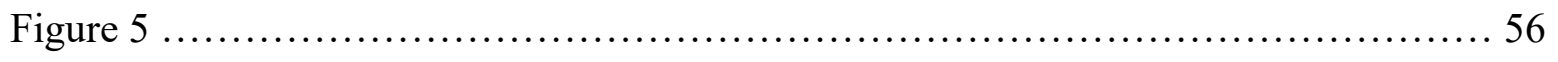

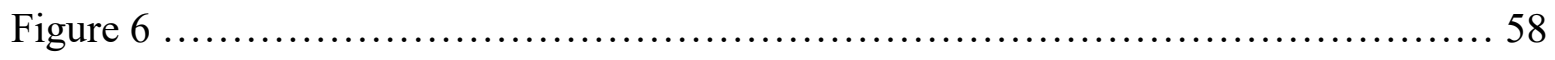


M.Sc. Thesis - J. El-Sayes; McMaster University - Kinesiology

\title{
List of Abbreviations
}

\author{
AMT - Active Motor Threshold \\ AURC - Area Under the Recruitment Curve \\ BDNF - Brain-Derived Neurotrophic factor \\ CBF - Cerebral Blood Flow \\ FDI - First Dorsal Interosseous \\ fMRI - functional Magnetic Resonance Imagery \\ GABA - Gamma-Aminobutyric Acid \\ ICF - Intracortical Facilitation \\ IGF-1 - Insulin like Growth Factor -1 \\ IPAQ - International Physical Activity Questionnaire \\ MEP - Motor Evoked Potential \\ NIBS - Non-invasive Brain Stimulation \\ NMDA - N-Methyl-D-aspartic Acid \\ RMT - Resting Motor Threshold \\ SICI - Short Interval Intracortical Inhibition \\ tDCS - Transcranial Direct Current Stimulation \\ TMS - Transcranial Magnetic Stimulation \\ TrkB - Tropomyosin-related Kinase B \\ VEGF - Vascular Endothelial Growth Factor
}


M.Sc. Thesis - J. El-Sayes; McMaster University - Kinesiology

\section{Declaration of Academic Achievement}

The entirety of this thesis has been written by Jenin El-Sayes and all experiments and TMS analyses were conducted by Jenin El-Sayes. Hormone and BDNF analyses were conducted by the CORE Lab at McMaster University and by Bernadeta Michalski, respectively. Dr. Aimee J. Nelson aided conception of the study and my fellow lab mates and collaborators Lauren Skelly, Claudia Turco, Dr. Chiara Nicolini, Mitchell Savoie, Diana Harasym, Mitchell Locke, and Stephen Toepp aided the collection of data. 
M.Sc. Thesis - J. El-Sayes; McMaster University - Kinesiology

\section{Chapter 1: Review of the Literature}




\section{Exercise-Induced Neuroplasticity in Humans}

Neuroplasticity is the ability of the central nervous system to change in response to the environment. These changes can occur at the molecular level, cellular level, and structural level, and can lead to behavioural changes in the long-term. Neuroplasticity is a key phenomenon, as it is crucial for development, learning, or recovery following neurological injury and disease.

It is well known that long-term aerobic exercise leads to improved quality of life, improved health, and reduced risk of cardiovascular disease (Goodman et al. 2016, Mang et al. 2013). Aerobic exercise can modulate many physiological systems involved in brain maintenance and neuroplasticity, such as neurotrophic and growth factors, cellular processes, and brain structure and function (see review El-Sayes et al., 2018). In the long-term, aerobic exercise results in improved cognitive (see review Stillman, Cohen, Lehman, \& Erickson, 2016) and motor function (see review Hübner \& Voelcker-Rehage, 2017). Importantly, acute aerobic exercise (i.e. a single session of exercise) can induce short-term neuroplasticity. The mechanisms underlying acute exercise-induced neuroplasticity are described below. These mechanisms include alterations in neurotrophic and growth factors, functional connectivity, and cognitive and motor function.

\section{Neurotrophic and Growth Factors}

Acute exercise modulates the concentration of peripheral brain-derived neurotrophic factor (BDNF). BDNF promotes neuroplasticity throughout development and adulthood (Monteggia et al., 2004; Poo, 2001) by increasing gliogenesis, neurogenesis, and synaptogenesis (see review ElSayes et al., 2018). Specifically, BDNF promotes neural stem cell differentiation into astrocytes 
M.Sc. Thesis - J. El-Sayes; McMaster University - Kinesiology

(Cheng et al., 2006) and stimulates neuronal cell proliferation and differentiation (Benraiss et al., 2001; Pencea et al., 2001; Zigova et al., 1998). BDNF regulates synapse formation and growth (Acheson et al., 1995; Binder and Scharfman, 2004) through strengthening of glutamatergic neurotransmission (Binder and Scharfman, 2004) and weakening of gamma-aminobutyric acid (GABAergic) neurotransmission (Binder and Scharfman, 2004; Kowiański et al., 2017). Following acute exercise, peripheral BDNF levels are increased (see review Huang, Larsen, RiedLarsen, Møller, \& Andersen, 2014). Studies testing different exercise intensities showed increases in BDNF following high intensity exercise (i.e. $10 \%$ above ventilatory threshold or $60 \%$ peak oxygen uptake) but not low intensity exercise (Ferris et al., 2007; Nofuji et al., 2012; Rojas Vega et al., 2012). While it has been suggested that peripheral BDNF levels may reflect brain BDNF levels (Krabbe et al., 2007), this remains unclear. BDNF is synthesized in both in the periphery (Wang et al., 2006) and in the central nervous system (Fujimura et al., 2002; Pan et al., 1998), but can only be obtained in vivo peripherally. BDNF is stored in platelets (Fujimura et al., 2002), and the analysis of BDNF levels is impacted by the completeness of blood clotting and the plasma isolation protocol (Fujimura et al., 2002). Further, ELISA kits used to analyze BDNF levels are susceptible to artifacts due to low specificity of BDNF antibodies and the presence of BDNF binding protein in the blood (Skornicka et al., 2002). Therefore, due to these confounding factors it remains unclear if obtained peripheral BDNF levels are a true reflection of brain BDNF.

BDNF levels are related to levels of insulin-like growth factor 1 (IGF-1) and vascular endothelial growth factor (VEGF). IGF-1 is crucial for normal brain development and maintenance (NietoEstévez et al., 2016; Sonntag et al., 2013), and increases neurogenesis, gliogenesis, and synaptogenesis (see review El-Sayes et al., 2018). IGF-1 promotes neural proliferation (Pérez- 
M.Sc. Thesis - J. El-Sayes; McMaster University - Kinesiology

Martín et al., 2010; Torres-Aleman et al., 1990) and differentiation into oligodendrocytes (Carson et al., 1993; Hsieh et al., 2004). IGF-1 also increases levels of tropomyosin-related kinase B (TrkB), the BDNF receptor, thereby increasing BDNF signaling (Cotman and Berchtold, 2002). Further, blocking IGF-1 prevents exercise-induced BDNF increases (Ding et al., 2006). IGF-1 and BDNF work together by converging on BDNF signaling as a common pathway to mediate exercise-induced neuroplasticity (Cotman and Berchtold, 2002). The effects of acute exercise on IGF-1 levels are inconsistent. Some studies have reported increases in IGF-1 following exercise (Cappon et al., 1994; Kraemer et al., 2004; Schwarz et al., 1996; Skriver et al., 2014), with greatest increases observed following high intensity exercise compare to lower intensities (Schwarz et al., 1996). However, other studies report no change in IGF-1 levels following exercise (Banfi et al., 1994; Griffin et al., 2011; Koistinen et al., 1996).

VEGF provides a vascular environment suitable for the growth of neurons (Louissaint et al., 2002) and promotes exercise-induced angiogenesis (Ding et al., 2006). Further, greater VEGF mRNA and protein levels are associated with exercise-induced increases in microvessel density (Ding et al., 2006). VEGF is also involved in neurogenesis, as exercise-induced increases in neurogenesis are abolished when VEGF increases are blocked (Fabel et al., 2003). IGF-1 is necessary for exercise-induced vessel remodeling (Lopez-Lopez et al., 2004) and BDNF stimulates VEGF transcription (Nakamura et al., 2011). VEGF levels are increased following acute exercise (see review El-Sayes et al., 2018). Therefore, acute exercise increases levels of neurotrophic and growth factors that mediate neuroplasticity induction. These increases indicate the capacity for acute exercise to induce short-term neuroplasticity. Increases in neurotrophic and growth factor 
levels mediate other short-term changes in the brain following exercise and eventually lead to the long-term changes induced by chronic exercise.

\section{Functional Connectivity and Cerebral Blood Flow}

Changes in brain function following acute exercise can be assessed using functional Magnetic Resonance Imagery (fMRI). Few studies have investigated the effects of acute exercise on functional connectivity in healthy adults. Rajab and colleagues (2014) showed an exercise-induced increase in functional connectivity in cortical loci associated with motor function and tactile processing. Activity was also increased in thalamic-caudate regions, which play a role in motor learning and reward (Rajab et al., 2014). Additionally, functional activity assessed during an executive function task is increased in the prefrontal (Li et al., 2014; Yanagisawa et al., 2010) and occipital (Li et al., 2014) cortices, while decreased in the cingulate (Li et al., 2014), frontal (Li et al., 2014; Yanagisawa et al., 2010), and parietal (MacIntosh et al., 2014) lobes following acute exercise. These changes in neural activity were accompanied by exercise-induced improvements in cognitive performance (Yanagisawa et al., 2010), but not always (Li et al., 2014). Changes in

neural activity are likely mediated by increases in cerebral blood flow $(\mathrm{CBF})$ observed following acute exercise (see review El-Sayes et al., 2018). Therefore, molecular changes induced by acute exercise are also observable on a circuit level.

\section{Cognitive and Motor Function}

Acute exercise has functional benefits as seen by improvements in cognitive and motor function. A single session of aerobic exercise reduces reaction time (Ozyemisci-Taskiran et al., 2008), 
improves memory and planning (Nanda et al., 2013) and improves working memory (Weng et al., 2015). Acute exercise also improves motor skill acquisition (Skriver et al., 2014; Snow et al., 2016;

Statton et al., 2015), retention (Roig et al., 2012; Skriver et al., 2014; R. Thomas et al., 2016; Richard Thomas et al., 2016b) and motor memory (Mang et al., 2016b; Roig et al., 2012; Richard Thomas et al., 2016a). These findings support the capacity for acute exercise to promote positive behavioural outcomes.

\section{Summary}

It is evident that acute aerobic exercise is capable of inducing neuroplasticity. Acute exercise increases key neurotrophic and growth factors, such as BDNF and VEGF. These increases likely drive changes in functional connectivity observed following a single session of exercise. The exercise-induced increases in cerebral blood flow help sustain this increased neural activity by providing neurons with oxygen and nutrients (see review El-Sayes et al., 2018). Last, increases in neural activity and connectivity likely mediate the improvements in cognitive and motor function observed following acute exercise. As such, acute exercise is a viable tool to induce neuroplasticity in both experimental and clinical settings.

\section{Transcranial Magnetic Stimulation: Assessing Neuroplasticity}

Transcranial magnetic stimulation (TMS) is a non-invasive tool used to stimulate the brain and assess the excitability of the motor cortex and corticospinal tract (Hallett, 2007). TMS produces a magnetic field that induces a perpendicular electric field within the cortex, exciting cortical neurons (Hallet, 2000; Hallett, 2007) (Figure 1). TMS depolarizes superficial pyramidal neurons 
M.Sc. Thesis - J. El-Sayes; McMaster University - Kinesiology

that have excitatory synapses onto corticospinal neurons (Di Lazzaro et al., 2012). The corticospinal volleys travel down the spinal cord and synapse onto alpha motor neurons creating an excitatory post-synaptic potential (EPSP) in the spinal motorneuron pool. If the EPSP depolarizes the spinal motorneuron pool, action potentials will be generated resulting in a measurable motor response from the targeted muscle (Siebner and Rothwell, 2003). The motor response from the muscle of interest is recorded using electromyography (EMG). As such, TMS allows for the investigation of specific muscle representations within the motor cortex by stimulating descending corticospinal neurons to the target muscle. See Table 1 for a summary of TMS measures. 


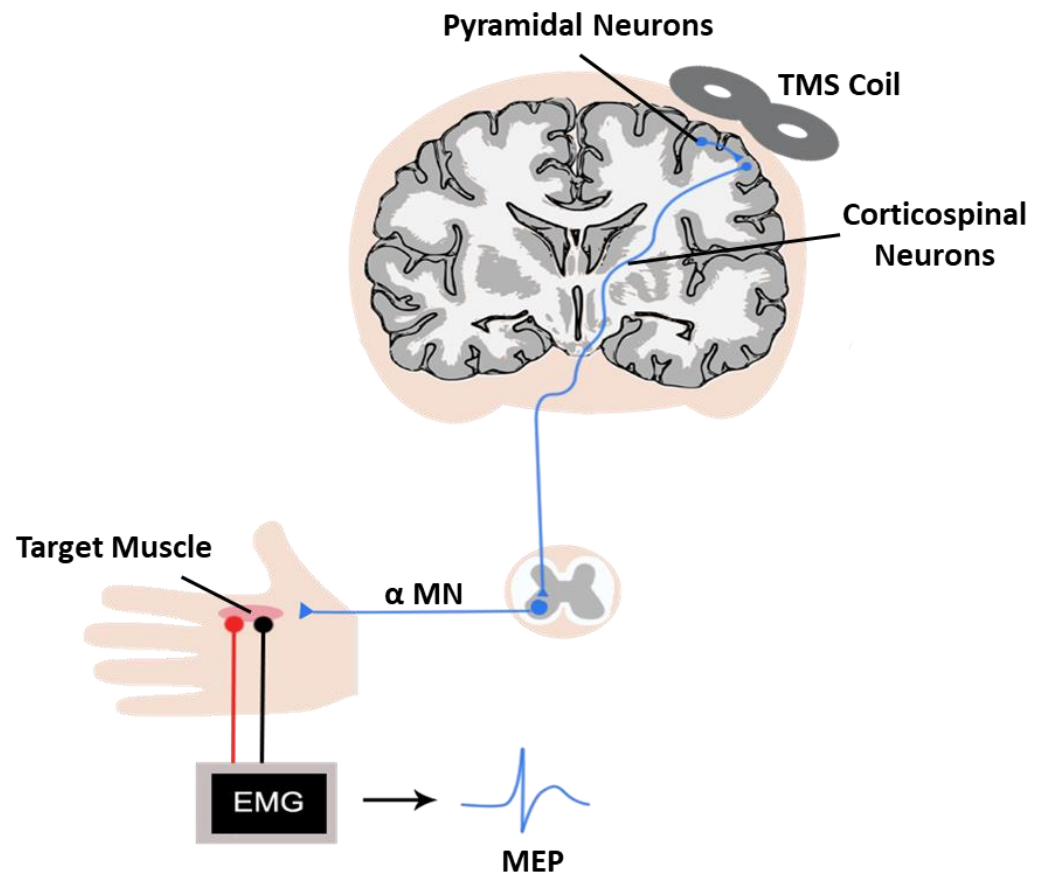

Figure 1. Pathway of TMS stimulation along the corticospinal tract: TMS excites pyramidal neurons, leading to excitation of corticospinal neurons, which synapse onto alpha motorneurons. Excitation of alpha motorneurons leads to a response in the target muscle, known as a MEP, which is recorded using EMG. TMS: Transcranial Magnetic Stimulation, a MN: Alpha Motorneuron, MEP: Motor Evoked Potential, EMG: Electromyography.

Motor Threshold

TMS can be used to acquire motor thresholds, which are measures of cortical excitability and indicative of voltage-gated sodium channel activity (Ziemann et al., 2014). Voltage-gated sodium channels are responsible for the generation of action potentials in neurons. Motor thresholds are dependent on the activity of synaptic relays in the cortex and the spinal cord (Siebner and Rothwell, 2003). Antiepileptic drugs blocking voltage gated sodium channels increase motor threshold (i.e. 
M.Sc. Thesis - J. El-Sayes; McMaster University - Kinesiology

reduce excitability), while NMDA receptor antagonists decrease motor threshold (see review Ziemann et al. 2014).

Resting motor threshold (RMT) is defined as the minimum TMS stimulus intensity required to elicit a motor evoked potential (MEP) while the muscle is at rest. It is obtained by determining the minimum TMS stimulus intensity that elicits an MEP amplitude of $50 \mu \mathrm{V}$ or greater in 5 out of 10 consecutive trials (Rossi et al., 2009). Active motor threshold (AMT) is defined as the minimum TMS stimulus intensity required to elicit a MEP while the muscle is maintaining an isometric contraction. It is obtained by determining the minimum TMS stimulus intensity required to elicit a peak-to-peak MEP amplitude of $200 \mu \mathrm{V}$ or greater in 5 out of 10 consecutive trials while participants maintain a percentage of their maximum voluntary contraction of the target muscle (Rossi et al., 2009; Siebner and Ziemann, 2014).

\section{Motor Evoked Potentials}

MEPs are electrical potentials recorded from a muscle following delivery of TMS to the motor cortex. The MEP amplitude quantifies the level of corticospinal excitability, such that larger amplitudes indicate greater corticospinal excitability (Bestmann and Krakauer, 2015). Positive allosteric modulators of $\mathrm{GABA}_{\mathrm{A}}$ receptors decrease MEPs (see review Ziemann et al. 2014). Comparatively, serotonin and noradrenaline agonists increase MEP amplitude (see review Ziemann et al. 2014). Therefore, MEP amplitude elicited by TMS reflects the activation of corticospinal neurons through a network of excitatory circuits that are mediated by inhibitory synaptic connections (see review Ziemann et al. 2014). A common assessment of MEPs is a 
recruitment curve, which displays the growth of MEP amplitude with TMS stimulus intensity in a sigmoidal function (Figure 2) (Devanne et al., 1997; Hess et al., 1987; Möller et al., 2009). As the TMS intensity increases, the MEP amplitude also increases as more upper and lower motorneurons are recruited. At higher TMS intensities the curve will reach a plateau, indicating that all available motor neurons have been recruited using TMS.

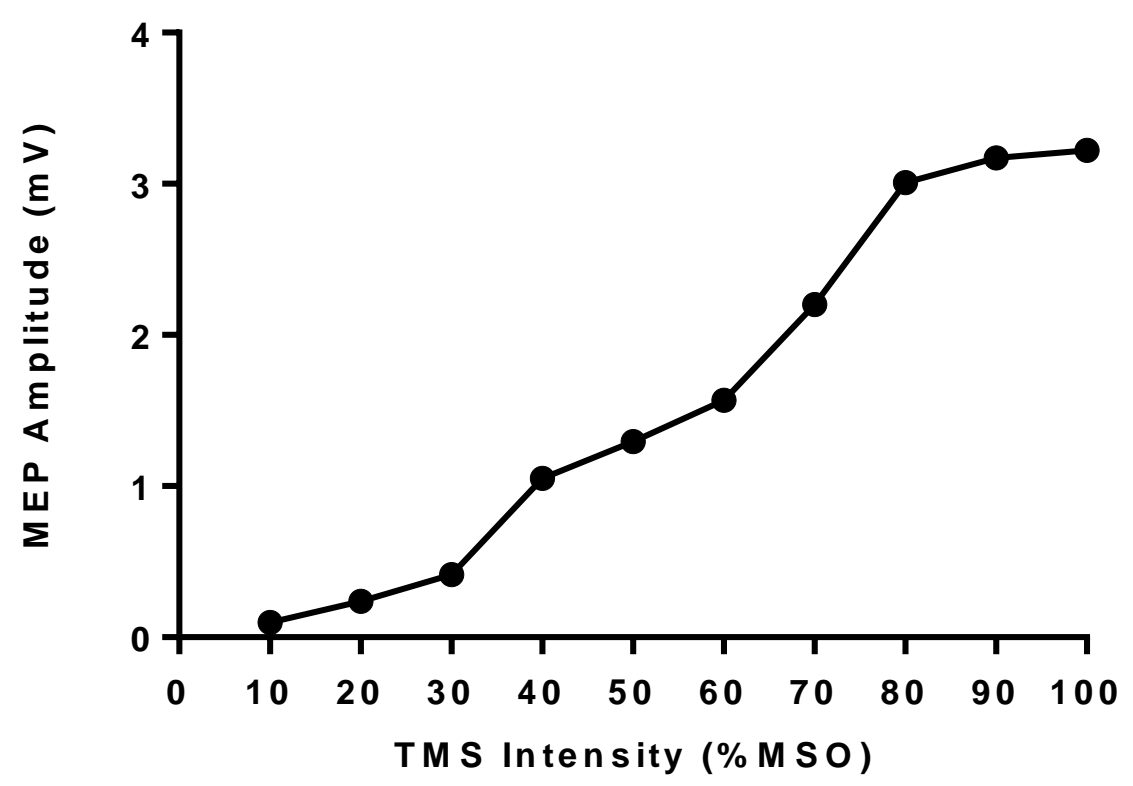

Figure 2. MEP Recruitment Curve: MEP amplitude increases as TMS intensity increases. Curve reaches a plateau when all motorneurons have been recruited. MEP: Motor Evoked Potential; TMS: Transcranial Magnetic Stimulation, MSO: Maximum Stimulation Output. Figure created for illustration purposes only.

\section{Short Interval Intracortical Inhibition}

Short interval intracortical inhibition (SICI) is thought to represent short lasting inhibitory postsynaptic potentials (IPSPs) in corticospinal neurons and is a result of activity in the intracortical inhibitory circuits utilizing GABA as a neurotransmitter (see review Ziemann et al. 2014). 
M.Sc. Thesis - J. El-Sayes; McMaster University - Kinesiology

Specifically, it is an indirect measure of $\mathrm{GABA}_{\mathrm{A}}$ circuitry in the motor cortex (Maruyama et al., 2006; Singh and Staines, 2015; Ziemann et al., 2014). SICI is measured using a paired-pulse TMS protocol that delivers a subthreshold conditioning stimulus (CS) followed by a suprathreshold test stimulus (TS) 1-5 ms later (Kujirai et al., 1993). The CS results in the inhibition of the TS-evoked MEP.

Table 1. Summary of TMS Dependent Measures

\begin{tabular}{|c|c|c|}
\hline TMS Dependent Measure & Receptor Activity & Common Stimulation Parameters \\
\hline Motor Thresholds (RMT, AMT) & Voltage Gated $\mathrm{Na}^{+}$ & $\begin{array}{l}\text { RMT: } \mathrm{MEP} \geq 50 \mu \mathrm{V} \text { in } 5 \text { out of } 10 \text { trials } \\
\text { (at rest) } \\
\text { AMT: MEP } \geq 200 \mu \mathrm{V} \text { in } 50 \text { out of } 10 \\
\text { trials (during tonic contraction) }\end{array}$ \\
\hline $\begin{array}{l}\text { Motor Evoked Potential Recruitment } \\
\text { Curves }\end{array}$ & $\mathrm{NMDA}, \mathrm{GABA}_{\mathrm{A}}$ & $\begin{array}{l}\text { Single-pulse TMS at increasing } \\
\text { stimulation intensities (e.g. } 90-200 \% \\
\text { RMT or } 10-100 \% \text { MSO) }\end{array}$ \\
\hline Short Interval Intracortical Inhibition & $\mathrm{GABA}_{\mathrm{A}}$ & $\begin{array}{l}\text { Paired-pulse TMS - ISI: } 2 \mathrm{~ms}, \mathrm{TS}: 1 \mathrm{mV} \text {, } \\
\text { CS: } 80 \text { or } 90 \% \text { AMT }\end{array}$ \\
\hline \multicolumn{3}{|c|}{$\begin{array}{l}\text { Receptor activity: Ziemann and others } 2014 ; \mathrm{Na}^{+}: \text {sodium ions; } N M D A: N \text {-Methyl-D-aspartic acid; } \\
\text { GABA: Gamma-Aminobutyric acid; ISI: interstimulus interval; TS: test stimulus; CS: conditioning } \\
\text { stimulus; RMT: resting motor threshold; AMT: active motor threshold; MSO: Maximum Stimulator } \\
\text { Output. }\end{array}$} \\
\hline
\end{tabular}

TMS to Measure Exercise-Induced Neuroplasticity

TMS can be used to assess short-term changes in neurotransmitter receptor activity that follows acute exercise. No changes in RMT (Lulic et al., 2017; Smith et al., 2014) or AMT (Lulic et al., 2017) are observed following acute exercise. MEP amplitude has been shown to increase (Lulic et al., 2017) or not change following exercise (Singh et al., 2014; Smith et al., 2014; Stavrinos and Coxon, 2017; Yamaguchi et al., 2012). Discrepancies between findings may be due to participant demographics, as physically active individuals show increases in MEPs following exercise while 
inactive individuals do not (Lulic et al., 2017). ICF (i.e. glutamatergic neurotransmission) is increased (Singh et al., 2014) or decreased (Lulic et al., 2017) following exercise. Discrepancies here are likely due to differences in exercise and TMS protocols. Long-interval intracortical inhibition (LICI), a measure of $\mathrm{GABA}_{\mathrm{B}}$ neurotransmission, is reduced following exercise (Mooney et al., 2016), indicating reduced $\mathrm{GABA}_{\mathrm{B}}$ receptor activity following exercise. Further, SICI (i.e. $\mathrm{GABA}_{\mathrm{A}}$ neurotransmission) is reduced following acute exercise (Lulic et al., 2017; Singh et al., 2014; Smith et al., 2014; Stavrinos and Coxon, 2017), indicating reduced GABA receptor activity following exercise. These findings indicate it is feasible to assess the effects of acute exercise on receptor activity using TMS.

\section{Factors Impacting Exercise-Induced Neuroplasticity}

Although acute exercise induces neuroplasticity, several factors may contribute to the direction and magnitude of neuroplasticity induction. Fitness level, biological sex, ovarian hormones, and cortisol have all been shown to impact neuroplasticity induced by non-invasive brain stimulation (NIBS). These factors should be considered in experimental settings to reduce the variability within and between studies. Further, these factors may impact the success of rehabilitation exercise protocols attempting to induce neuroplasticity via acute exercise, and therefore should be considered in clinical settings.

\section{Fitness Level}

Physically active individuals show greater NIBS-induced and exercise-induced neuroplasticity compared to sedentary individuals. Following excitatory NIBS, fit individuals show the expected increase in corticospinal excitability, assessed via MEPs, while sedentary individuals do not 
M.Sc. Thesis - J. El-Sayes; McMaster University - Kinesiology

(Cirillo et al., 2009). Further, only individuals participating in greater amounts of physical activity show increases in corticospinal excitability following 20 minutes of moderate intensity cycling (Lulic et al., 2017) (Figure 3). The enhanced propensity for neuroplasticity in fit individuals is likely attributed to the long-term changes observed following chronic exercise (e.g. increases in neurotrophic factors, brain volume, and cerebral blood flow). Increased central circulation of neurotrophic and growth factors upregulate neurogenesis, synaptogenesis, gliogenesis, and angiogenesis (El-Sayes et al., 2018). Through these processes, aerobic exercise increases grey and white matter volume, leading to increased efficiency of neural activity and communication (ElSayes et al., 2018). These changes are sustained by increased cerebrovascular function, which provides adequate nutrients to support the increase in brain volume and activity (El-Sayes et al., 2018). 


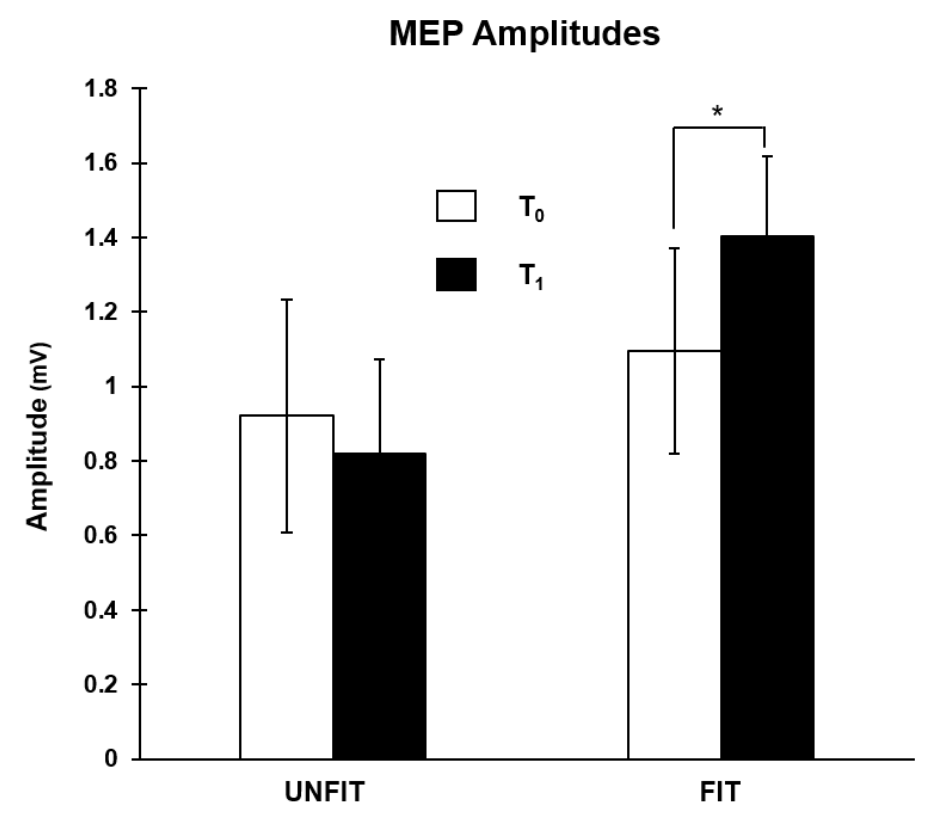

Figure 3. Motor evoked potential (MEP) amplitude (i.e. corticospinal excitability) increases following exercise only in highly physically active individuals but not low to moderately active individuals. Figure adapted with permission from Lulic et al. 2017, PLOS One.

\section{Biological Sex}

Females have greater neuroplasticity induction following NIBS compared to males (see review Ridding and Ziemann 2010). For example, females show a greater reduction in MEP amplitude following cathodal transcranial direct current stimulation (ctDCS) to the motor cortex compared to males (Kuo et al., 2006). Females also show greater neuroplasticity induction following anodal tDCS (atDCS) to the visual cortex compared to males (Chaieb et al., 2008). It is likely that females will show greater exercise-induced neuroplasticity, as studies with a larger proportion of female participants than males show greater improvements in cognitive function following aerobic training (see reviews Barha, Davis, et al. 2017; Colcombe and Kramer 2003; Barha, Galea, et al. 2017). Further, although both sexes reportedly have similar levels of peripheral BDNF 
M.Sc. Thesis - J. El-Sayes; McMaster University - Kinesiology

(Lommatzsch et al., 2005; Piccinni et al., 2008), females have higher brain levels of BDNF expression compared to males (Michalski et al., 2015). Following aerobic training, females show increases in peripheral BDNF levels and improvements in executive function, while males did not (Barha et al., 2017d). Sex differences in neuroplasticity induction may be mediated by structural and functional differences between sexes. Females have greater grey matter volume, cerebral blood flow, and baseline neural activity compared to males (see review Cosgrove, Mazure, and Staley 2007). Taken together, these findings suggest that exercise-induced neuroplasticity is likely greater in females compared to males.

\section{Menstrual Cycle and Ovarian Hormones}

In females, neuroplasticity induction is greater when estradiol is high (Inghilleri et al., 2004). Neuroplasticity induced by NIBS is greater during ovulation compared to during menstruation (Inghilleri et al., 2004). Neuroplasticity induced by exercise may also be greater when estradiol levels are higher. In the CNS, estradiol is excitatory in nature, as it enhances N-methyl-D-aspartate (NMDA) receptor activity and blocks $\mathrm{GABA}_{\mathrm{A}}$ receptor activity (Amin et al., 2006). In animals, estradiol increases BDNF expression and protein levels (see review Sohrabji and Lewis, 2006). Progesterone exerts an inhibitory effect in the CNS through its metabolite, allopreganone, which modulates the activity of $\mathrm{GABA}_{\mathrm{A}}$ receptors (see review Guennoun et al., 2015). Allopreganone enhances inhibition by prolonging the opening of chloride channels in $\mathrm{GABA}_{\mathrm{A}}$ receptors (see review Guennoun et al., 2015). Further, greater levels of short interval intracortical inhibition

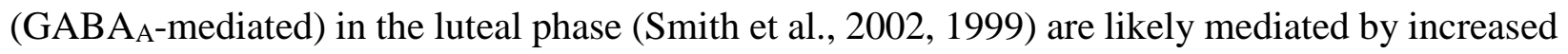
progesterone levels. By altering receptor function, estradiol and progesterone may impact neuroplasticity induction. 
BDNF and glutamate concentrations also fluctuate across the menstrual cycle, which may impact neuroplasticity induction. Plasma (Begliuomini et al., 2007; Cubeddu et al., 2011; Pluchino et al., 2009) and platelet (Lommatzsch et al., 2005) BDNF levels are greater in the luteal phase compared to the follicular phase. Further, estradiol levels are positivity correlated with plasma BDNF (Begliuomini et al., 2007; Pluchino et al., 2009), as estradiol can induce BDNF mRNA and protein synthesis (Carbone and Handa, 2013). Glutamate concentrations are lower in the luteal phase compared to the follicular phase (Batra et al., 2008; Zlotnik et al., 2011). These variations in BDNF and glutamate concentrations likely impact the propensity for neuroplasticity induction across the menstrual cycle.

\section{Cortisol}

Cortisol reduces the propensity for neuroplasticity induced by NIBS. Previous work shows that cortisol administration impedes increases in corticospinal excitability induced by paired associative stimulation (Sale et al., 2008). Further, cortisol administration reduces hippocampal activity (Lovallo et al., 2010), memory retrieval (de Quervain et al., 2003) and cerebral blood flow (de Quervain et al., 2003; Strelzyk et al., 2012). The impairment of neuroplasticity induction by cortisol is likely driven by its hyperpolarizing effects, as cortisol has been shown to increase GABA $_{A}$-mediated inhibition (Wotton et al., 2018). Therefore, it is likely that increases in cortisol due to exercise may also impede exercise-induced neuroplasticity. However, cortisol levels are increased following high intensity exercise (i.e. $80 \%$ maximal heart rate or $80 \% \mathrm{VO}_{2}$ peak) but not low or moderate intensity (i.e. $<57 \%$ maximal heart rate or $<60 \% \mathrm{VO}_{2}$ peak) (Hötting et al., 2016; VanBruggen et al., 2011). 
M.Sc. Thesis - J. El-Sayes; McMaster University - Kinesiology

\section{Conclusions and Unanswered Questions}

The beneficial effects of aerobic exercise are well understood and described, and there is now evidence that exercise is also capable of altering activity within the primary motor cortex. Several factors, such as fitness level, are known to impact exercise-induced neuroplasticity (see review ElSayes et al., 2018). However, not all factors are well understood. Evidence suggests that neuroplasticity induction via non-invasive brain stimulation is dependent on biological sex and ovarian hormones. It remains unclear whether exercise-induced neuroplasticity is impacted by these factors. Therefore, this research aimed to determine whether biological sex and ovarian hormones impact exercise-induced neuroplasticity. Information from this research may be used to improve the success of rehabilitation approaches that aim to increase corticospinal output to impaired muscles, such as following stroke. 
M.Sc. Thesis - J. El-Sayes; McMaster University - Kinesiology

\section{Chapter 2: Goals of Thesis and Significance}


M.Sc. Thesis - J. El-Sayes; McMaster University - Kinesiology

The overall purpose of the thesis is to investigate if biological sex and ovarian hormones impact the propensity for exercise-induced short-term neuroplasticity. This thesis used TMS to assess changes in neuronal output to the hand muscles following moderate intensity continuous cycling. Lower limb exercise has been shown to alter neuronal output to muscles of the hand/arm (Cirillo et al., 2009; Coco et al., 2016; Garnier et al., 2017; Kumpulainen et al., 2015; Lulic et al., 2017; McDonnell et al., 2013; McGregor et al., 2013; Mooney et al., 2016; Neva et al., 2017; Singh et al., 2014; Smith et al., 2014; Stavrinos and Coxon, 2017) as well as lower limb muscles (Harel et al., 2015; Jubeau et al., 2014; Motl and Dishman, 2003; Racinais et al., 2007; Sidhu et al., 2012; Yamaguchi et al., 2012). A goal of rehabilitation protocols (e.g. following stroke or spinal cord injury) is to increase neuronal output to the hand muscles to promote functional recovery. This study reveals differences in exercise-induced neuroplasticity of the primary motor cortex as a function of biological sex but not ovarian hormones, assessed via menstrual cycle phase. Therefore, this thesis provides important considerations for research and rehabilitation protocols aiming to induce neuroplasticity via acute exercise.

\section{Significance}

Acute aerobic exercise can be used to prime the motor cortex for plasticity, leading to improvements in motor learning (Snow et al. 2016) and increased response to non-invasive brain stimulation (NIBS) (Singh, Neva and Staines 2014b, McDonnell et al. 2013, Mang et al. 2014, Mang et al. 2016). Evidence suggests that the propensity for NIBS-induced neuroplasticity is affected by biological sex (Ridding and Ziemann, 2010) and ovarian hormones (Inghilleri et al. 2004). This research reveals that biological sex also impacts neuroplasticity induced by a single session of aerobic exercise, while ovarian hormones do not. 
M.Sc. Thesis - J. El-Sayes; McMaster University - Kinesiology

The research serves two main purposes: First, it reveals new neuroscience information about the effects of biological sex and ovarian hormone fluctuations on exercise modulation of the neural control of the upper limb. Second, the information obtained can be used for future comparison with special populations such as individuals who have suffered a stroke to determine whether sex and ovarian hormones impact the success of exercise protocols used in a clinical setting. Ultimately, the results obtained may be used to assist with developing new therapeutic interventions aimed at improving upper limb control following neurological injury. 
M.Sc. Thesis - J. El-Sayes; McMaster University - Kinesiology

\section{Chapter 3: Hypotheses}




\section{Biological Sex Effects}

It was hypothesized that corticospinal excitability, assessed via MEPs, will increase following acute exercise. The increase in MEPs would be greater in females than males, as females show greater neuroplasticity induction via non-invasive brain stimulation (Ridding and Ziemann, 2010).

\section{Ovarian Hormone Effects}

Within females, it was hypothesized that exercise-induced MEP increases will be greater in the follicular phase compared to the luteal phase of the menstrual cycle. Previous research has shown greater neuroplasticity induction via non-invasive brain stimulation during ovulation compared to menstruation (Inghilleri et al., 2004). Second, it was hypothesized that reductions in cortisol inhibition (i.e. SICI) will be greater in the follicular phase compared to the luteal phase. This is due to higher levels of progesterone in the luteal phase, which bind to $\mathrm{GABA}_{\mathrm{A}}$ receptors (Guennoun et al., 2015), likely blocking the expected reductions in SICI. 
M.Sc. Thesis - J. El-Sayes; McMaster University - Kinesiology

\section{Chapter 4: Experiment - The Effects of Biological Sex and Ovarian Hormones on Exercise-Induced Neuroplasticity}




\title{
M.Sc. Thesis - J. El-Sayes; McMaster University - Kinesiology
}

\begin{abstract}
Acute aerobic exercise induces neuroplasticity in part by increasing excitability and reducing inhibition within the motor cortex, as assessed via transcranial magnetic stimulation (TMS). It remains unknown if biological sex and ovarian hormones impact exercise-induced neuroplasticity. The present study investigated the effects of biological sex and ovarian hormones on neuroplasticity induced by acute aerobic exercise. Twenty-eight individuals (fourteen females, fourteen males) participated in two sessions in which TMS measures were acquired before and following 20 minutes of moderate intensity acute cycling at $65-70 \%$ of maximal heart rate. Females were tested in the follicular ( day 7) and luteal ( day 21) phases of the menstrual cycle. Males were tested on two sessions separated by $\sim 14$ days. TMS measures included motor evoked potential (MEP) recruitment curves and short interval intracortical inhibition (SICI). Measures of estradiol, progesterone, testosterone, and brain-derived neurotrophic factor (BDNF) were obtained pre-exercise on both testing occasions. Results indicate that MEP recruitment curves increased following exercise in females but not males, and this increase was associated with higher levels of serum BDNF such that higher serum BDNF levels were associated with a greater percent change in MEPs. Furthermore, there was a trend towards higher BDNF levels in the luteal phase compared to the follicular phase. SICI was unaltered by exercise in both sexes. Therefore, biological sex impacts the propensity for exercise-induced neuroplasticity and should be considered in future research. Results did not vary across the menstrual cycle, indicating that ovarian hormones do not impact the propensity for neuroplasticity.
\end{abstract}


M.Sc. Thesis - J. El-Sayes; McMaster University - Kinesiology

\section{Significance Statement}

Exercise can be used to prime the motor cortex for neuroplasticity, leading to improvements in motor learning and increased response to non-invasive brain stimulation (NIBS). Evidence suggests that the propensity for NIBS-induced neuroplasticity is influenced by biological sex and ovarian hormones. This research revealed that biological sex, but not ovarian hormones, influence neuroplasticity induced by a single session of aerobic exercise. These findings may be used to assist with developing new therapeutic interventions aimed at improving upper limb control following neurological injury. 


\section{Introduction}

Acute aerobic exercise can induce neuroplasticity and, as such, offers a cost-effective approach to rehabilitation and improvements in quality of life. It is unknown if exercise-induced neuroplasticity is influenced by biological sex; however, evidence suggests that a difference between sexes may exist. Increases in brain-derived neurotrophic factor (BDNF) and improvements in cognitive function following aerobic training are greater in females compared to males (Barha et al., 2017b). Non-invasive brain stimulation (NIBS) induces greater neuroplasticity in females compared to males (see review Ridding and Ziemann, 2010). Females have greater grey matter volume, cerebral blood perfusion, and neural activation than males (Cosgrove et al., 2007), suggesting that males and females may respond differently to neuroplasticity inducing interventions.

Ovarian hormones may also influence exercise-induced neuroplasticity. NIBS-induced increases in motor evoked potentials (MEPs) are only observed on day 14 of the menstrual cycle, when estradiol levels are high, but not on day 1, when estradiol levels are low (Inghilleri et al., 2004). This is likely due to the excitatory effects of estradiol that enhances N-methyl-D-aspartate (NMDA) receptor activity and blocks $\mathrm{GABA}_{\mathrm{A}}$ activity (Amin et al., 2006). In contrast, in the luteal phase, greater progesterone levels increase cortical inhibition, as progesterone enhances $\mathrm{GABA}_{\mathrm{A}}$ activity (Guennoun et al., 2015).

TMS measures of corticospinal excitability and cortical inhibition offer an opportunity to explore the propensity for exercise-induced neuroplasticity. An acute bout of aerobic exercise increases 
MEP amplitude (Lulic et al., 2017) and reduces short interval intracortical inhibition (SICI) (Lulic et al., 2017; Singh et al., 2014; Smith et al., 2014). The present study investigated the influence of sex and ovarian hormones on corticospinal excitability and intracortical inhibition before and following an acute bout of aerobic exercise in recreationally active adults. It was hypothesized that females would show greater exercise-induced increases in MEPs compared to males, as females show a greater response to neuroplasticity induced by non-invasive brain stimulation (Ridding and Ziemann, 2010). In females, increases in exercise-induced MEP and SICI reductions were expected to be greater when progesterone levels are low during the follicular phase. Results indicate that acute exercise increased corticospinal excitability in females but not males, in agreement with the effects of NIBS. No change in SICI was observed following exercise, nor did neuroplasticity vary with the phase of the menstrual cycle.

\section{Methods}

\section{Participants}

Twenty-eight individuals (Male: $\mathrm{n}=14,21.4 \pm 2.3$ years; female: $\mathrm{n}=14,20.6 \pm 2.1$ years) participated in three sessions. There were no age differences between sexes (Mann Whitney: $\mathrm{p}=$ 0.412). All individuals were right-hand dominant as determined by the modified version of the Edinburgh Handedness Scale (Oldfield, 1971) and had no known history of neurological disease. Individuals on hormonal contraceptives or hormonal supplements were excluded. Only recreationally active individuals (i.e. those with cardiorespiratory fitness ( $\mathrm{VO}_{2}$ peak) in the "fair" or higher category defined by the Canadian Society for Exercise Physiology) were included in the study, as physically active individuals show greater propensity for neuroplasticity (Cirillo et al., 2009; Lulic et al., 2017). All participants were screened for contraindications to TMS (Rossi et al., 
M.Sc. Thesis - J. El-Sayes; McMaster University - Kinesiology

2009) and exercise (Physical Activity Readiness Questionnaire; Warburton et al., 2011). Written informed consent was obtained prior to participation. This study was approved by the McMaster Research Ethics Board and the Hamilton Integrated Research Ethics Board and conformed to the Declaration of Helsinki.

\section{Experimental Design}

In the first session, fat-free mass (FFM) was determined through air displacement plethysmography (Bod Pod®, COSMED Inc., Concord, CA, USA), after a period of fasting (minimum of 3 hours). Following a small meal, fitness level was assessed using a maximal oxygen uptake test ( $\mathrm{VO}_{2}$ peak test) completed on an electronically braked cycle ergometer (Lode Excalibur Sport V 2.0, Groningen, the Netherlands) and an on-line gas collection system (Moxus modular oxygen uptake system, AEI Technologies, Pittsburg, PA, USA). For the $\mathrm{VO}_{2}$ peak test, a 2 minute warm-up at $50 \mathrm{~W}$ was followed by an increase in the work intensity at a rate of $1 \mathrm{~W}$ every 2 seconds until volitional fatigue occurred or until participants could no longer cycle at 60 r.p.m. The $\mathrm{VO}_{2}$ peak corresponded to the highest value achieved over a 30 second period. For each participant, the $\mathrm{VO}_{2}$ peak was calculated relative to FFM (equation below). Males and females were matched for fitness level based on their $\mathrm{VO}_{2}$ peak relative to FFM, according to established practice for making sex-based comparisons (Tarnopolsky, 2008).

$$
\mathrm{VO}_{2} \text { peak }(\mathrm{mL} / \mathrm{kgFFM} / \mathrm{min})=\frac{\mathrm{VO}_{2} \text { peak }\left(\frac{\mathrm{mL}}{\mathrm{min}}\right)}{\text { Fat-free mass }(\mathrm{kg})}
$$

Sessions 2 and 3 were conducted in the morning (starting between 9-11am) and followed the experimental timeline in Figure 1. Females were tested during the mid-follicular (days 6 to 9) and 
M.Sc. Thesis - J. El-Sayes; McMaster University - Kinesiology

mid-luteal (days 19 to 23) phases of the menstrual cycle. The order of testing days (i.e. follicular vs. luteal) was randomized among participants. Males were also tested on two occasions approximately 14 days apart. Physical activity levels were assessed using the International Physical Activity Questionnaire (IPAQ; Craig et al., 2003) to confirm that physical activity levels were similar between the two experimental sessions. Each session was divided into two time blocks: T0 (pre-exercise) and T1 (10 minutes post-exercise). At T0, fasted blood samples were collected from the antecubital vein to quantify levels of estradiol, progesterone, testosterone, and serum BDNF. BDNF levels were assessed to determine differences between sexes and across the menstrual cycle, and to determine if exercise-induced changes in TMS measures are associated with BDNF levels. All other dependent measures (listed below) were obtained at T0 and T1. The order of dependent measure acquisition was pseudorandomized using the William Square Counterbalance and held constant between Sessions 2 and 3 within a participant.

\section{Acute Exercise Intervention}

Participants completed lower limb cycling on an electronically braked cycle ergometer (Ergo Race, Kettler, Germany). The exercise protocol included 5 minutes of warm up, 20 minutes of moderate intensity continuous cycling at $65-70 \%$ of maximum heart rate $\left(\mathrm{HR}_{\max }\right)$ (Garber et al., 2011), followed by 5 minutes of cool down. For the 20 minutes of moderate intensity cycling, participants were instructed to cycle at a self-selected pace between 70-90 r.p.m. as indicated on the ergometer display. This pace was kept constant during the two experimental sessions. Throughout the exercise, EMG activity of the FDI muscle $\left(\mathrm{EMG}_{\text {exercise }}\right)$ was recorded to ensure that the FDI muscle (the target muscle for TMS-evoked measures) was inactive. Heart rate was monitored using telemetry (Polar A3, New York, USA) to obtain continuous data for the 30 minutes exercise period 
M.Sc. Thesis - J. El-Sayes; McMaster University - Kinesiology

and the 10 minute rest period following the exercise. Ratings of perceived exertion (RPE) were obtained every 2 minutes during the 20 minute intervention using the 0-10 Borg scale (Borg, 1990) to assess the subjective levels of fatigue. Salivary cortisol levels were assessed before and immediately following the exercise intervention to assess fatigue elicited by the exercise protocol.

\section{Electromyography Recording}

Electromyography (EMG) was recorded using surface electrodes (9 mm diameter $\mathrm{Ag}-\mathrm{AgCl})$ placed over the right first dorsal interosseous (FDI) muscle in a belly tendon montage. A wet ground electrode was placed around the forearm. EMG signals were amplified (x1000), bandpass filtered between $20 \mathrm{~Hz}$ and $2.5 \mathrm{kHz}$ (Intronix Technologies Corporation Model 2024F with Signal Conditioning; Intronix Technologies Corporation, Bolton, Canada), and digitized at $5 \mathrm{kHz}$ (Power1401, Cambridge Electronic Design, Cambridge, UK). EMG data were collected using Signal software version 6.02 (Cambridge Electronic Design, Cambridge, UK).

\section{Maximum Voluntary Contraction}

Participants completed three maximal isometric contractions of the right FDI against an immovable structure. Each contraction persisted for 5 seconds with a 30 second rest interval between trials. The largest EMG activity obtained from any of the three trials was defined as the maximum voluntary contraction (MVC) of FDI for an individual. The level of EMG activity corresponding to $10 \%$ MVC was displayed on an oscilloscope as a horizontal target line. Participants were required to match and maintain $10 \%$ MVC by contracting their right FDI during the acquisition of active motor threshold (AMT) (see below). 


\section{M.Sc. Thesis - J. El-Sayes; McMaster University - Kinesiology}

\section{M-Max}

Maximum M-wave (M-Max) was defined as the maximum response elicited from the right FDI following ulnar nerve stimulation at the wrist. Nerve stimulation was delivered using a bar electrode (cathode proximal) and a constant current stimulator (Digitimer DS7AH) delivering square wave pulses with a $200 \mu$ s pulse width. Stimulation intensity was increased by $1 \mathrm{~mA}$ upon each subsequent stimulation until the M-wave ceased to increase in 3 consecutive trials. The peakto-peak amplitude of the M-wave (mV) was defined as M-max.

\section{Transcranial Magnetic Stimulation}

Single and paired monophasic TMS pulses were delivered using a custom-built $50 \mathrm{~mm}$ diameter figure-of-eight branding coil connected to a Magstim Bistim stimulator (Magstim, Whitland, UK). The TMS coil was positioned 45 degrees in relation to the parasagittal plane to induce a posteriorto-anterior current in the cortex. The motor hotspot for the right FDI was determined within the left motor cortex and defined as the location that elicited consistent MEPs. The motor hotspot was digitally registered using Brainsight Neuronavigation (Rogue Research, Canada). All dependent measures were obtained from the right FDI. Resting motor threshold (RMT) and AMT were first acquired. RMT was defined as the lowest intensity required to evoke a MEP $\geq 50 \mu \mathrm{V}$ in 5 out of 10 consecutive trials in the relaxed FDI muscle (Rossi et al., 2009). AMT was defined as the lowest intensity required to evoke a $\mathrm{MEP} \geq 200 \mu \mathrm{V}$ in 5 out of 10 consecutive trials while participants maintained $~ 10 \%$ MVC in their FDI (Rossi et al., 2009). 
MEP recruitment curves were obtained at rest by delivering seven TMS pulses at $90-200 \%$ RMT in $10 \%$ increments in a randomized order (84 pulses total). SICI was tested using paired-pulse TMS at an interstimulus interval of $2 \mathrm{~ms}$ between the conditioning stimulus (CS) and test stimulus (TS). Two CS intensities (80\% and 90\% AMT) were tested, and the TS was set to evoke MEPs with peak-to-peak amplitudes of $\sim 1 \mathrm{mV}$ in the right FDI rest. For each CS intensity, twenty samples were acquired whereby equal numbers of unconditioned (10 $\left.\mathrm{MEP}_{\mathrm{TS}}\right)$ and conditioned (10 MEP $\left._{\text {CS-TS }}\right)$ trials were randomly delivered.

\section{Hormone, Cortisol, and BDNF Analyses}

Fasted blood was collected at T0 using red top BD Vacutainer® collection tubes (BD and Company, Frankin Lakes, NJ, USA). Tubes were left to clot at room temperature for $\sim 50$ minutes and subsequently centrifuged at 4000 r.p.m. for 10 minutes at $4^{\circ} \mathrm{C}$. The supernatant was aliquoted into vials and stored at $-80^{\circ} \mathrm{C}$. Saliva samples were obtained using Salivettes (Sarstedt, Nümbrecht, Germany) and centrifuged at 4000 r.p.m. for 10 minutes and stored at $-80^{\circ} \mathrm{C}$. The Hamilton Regional Medicine Program Core Laboratory quantified serum estradiol and progesterone levels using chemiluminescent microparticle immunoassay (Abbott Architect, Abbott Laboratories, Chicago, USA), serum testosterone using a chemiluminescent enzyme immunoassay (Immulite 2000; Siemens, Erlangen, Germany), and salivary cortisol using the Cortisol ELISA Kit (Enzo Life Sciences, New York, USA). Serum BDNF was analyzed using human BDNF DuoSet ELISA kit (R\&D Systems, Minneapolis, USA). 


\section{M.Sc. Thesis - J. El-Sayes; McMaster University - Kinesiology}

\section{Data Analyses}

All MEP and SICI trials were assessed for background muscle activity. Trials were excluded if the EMG activity $20 \mathrm{~ms}$ immediately before the TMS stimulus artifact exceeded two times the EMG activity in a $20 \mathrm{~ms}$ window at the beginning of the trial. The mean peak-to-peak MEP amplitude at each intensity $(90-200 \%$ RMT) of the recruitment curve was calculated by averaging the seven trials at each intensity. MEP recruitment curves were fitted to a Boltzmann sigmoid function (Thirugnanasambandam et al., 2015; van Kuijk et al., 2009), and all further analyses were conducted on the fitted curves. The $\underline{\text { Area }} \underline{\text { Under the fitted }} \underline{\text { Recruitment }} \underline{\text { Curve }}$ (AURC) was obtained by trapezoidal integration $25 \%$ above and below the inflection point. SICI was assessed by calculating the peak-to-peak amplitude of the unconditioned $\left(\mathrm{MEP}_{\mathrm{TS}}\right)$ and conditioned $\left(\mathrm{MEP} \mathrm{CS}_{\mathrm{C}}\right.$ TS) MEP at each CS intensity. The percentage of inhibition was calculated as the ratio of the conditioned over unconditioned MEP (MEP $\left.\mathrm{CS}_{\mathrm{TS}} / \mathrm{MEP}_{\mathrm{TS}}\right)$. AURC and SICI were normalized to MMax (i.e. AURC/M-Max, SICI/M-Max) at T0 and T1 to account for altered electrode conductance following the exercise (Abdoli-Eramaki et al., 2012). Percent change (across time) was calculated

for all dependent measures using the following formula: $\left(\frac{(\mathrm{T} 1-\mathrm{T} 0)}{\mathrm{T} 0}\right) \times 100$. Female data was organized into the appropriate phase (i.e. follicular, luteal). For each male participant, the data was organized to replicate the order of testing performed by their female match (i.e. follicular-match, luteal-match).

Group-level analyses included normality testing using the Shapiro-Wilk's test. Outliers were identified using IBM SPSS Software as data points 3 times above or below the interquartile range and were removed from further analyses (indicated in tables). Non-normally distributed data were $\log 10$ or square root transformed and assessed using parametric statistics. If these transformations 
M.Sc. Thesis - J. El-Sayes; McMaster University - Kinesiology

did not normalize the data, the data was ranked and assessed using a Conover's ANOVA (Conover and Iman, 1981). All data transformations are indicated in the tables. Post-hoc tests included Tukey's honestly significant difference test for normally distributed data and Wilcoxon SignedRank and Mann-Whitney $U$ tests for non-normally distributed data. Correlational analyses were run using Pearson's r for normally distributed data and Spearman's rho for non-normally distributed data. The significance level was set to $\mathrm{p} \leq 0.05$, and effect sizes were calculated using Hedge's g.

For AURC and SICI, data from males and females were analyzed separately using a two-way repeated-measures ANOVA with within-subject factors of PHASE (2 levels: Females: Follicular, Luteal; Males: Follicular-Match, Luteal-Match) and TIME (2 levels: T0, T1). If there was no effect of PHASE, the effects of TIME (i.e. exercise) were analyzed using a two-tailed paired t-test or Wilcoxon Signed Rank Test. Sexes were analyzed separately for AURC as baseline AURC, was higher in males compared to females, although this did not reach significance. Further, changes in SICI were for each sex were assessed separately as females have been shown to have greater SICI compared to males (Shibuya et al., 2016). RMT, AMT, M-Max, and salivary cortisol were assessed using three-way mixed ANOVAs with between-subjects factor SEX (2 levels: Male, Female) and within-subject factors of PHASE (2 levels: Females: Follicular, Luteal; Males: Follicular-Match, Luteal-Match) and TIME (2 levels: T0, T1). Estradiol, progesterone, testosterone, serum BDNF, and $\mathrm{EMG}_{\text {exercise }}$ were assessed using a two-way mixed ANOVA with between-subject factor SEX (2 levels: Male, Female) and within-subject factor PHASE (2 levels: Females: Follicular, Luteal; Males: Follicular-Match, Luteal-Match). 


\section{M.Sc. Thesis - J. El-Sayes; McMaster University - Kinesiology}

\section{Results}

All participants successfully completed the study. Descriptive characteristics for participants are presented in Table 1. Males and females were matched for cardiorespiratory fitness (unpaired ttest: $\mathrm{p}=0.489$ ). Females were tested on days $7.8 \pm 2.1$ (follicular) and $21.7 \pm 5.6$ (luteal) of their cycle. The two sessions were separated by $16.2 \pm 8.5$ days for females and $15.2 \pm 6.6$ days for males (Mann Whitney: $\mathrm{p}=0.349$ ). Physical activity levels, assessed via IPAQ, did not differ between the two experimental sessions for either sex $\left(\mathrm{F}_{(1,26)}=0.881, \mathrm{p}=0.356\right)$. Hormone and serum BDNF levels are provided in Table 2. The estradiol to progesterone ratio (E:P) in females was higher in the follicular phase compared to the luteal phase of the menstrual cycle (Tukey's: $p$ $<0.001)$. There was a trend towards greater serum BDNF in females in the luteal phase compared to the follicular phase (Tukey's: $p=0.057$; Figure 2). Previous reports also show greater plasma BDNF levels in the luteal phase compared to the follicular phase (Begliuomini et al., 2007; Cubeddu et al., 2011; Pluchino et al., 2009). The lack of a significant effect in this work may be due to low sample size ( $\mathrm{n}=14$ per group). A power calculation from previous work with similar effect sizes (0.74 in Pluchino et al., 2009 vs. 0.64 in this work) suggests a sample size of 17 participants is needed to show a significant finding. BDNF levels were not different between sessions for males (Tukey's: $\mathrm{p}=0.112$; Figure 2). Serum BDNF levels in females at each menstrual cycle phase were not different from levels in males (averaged over both sessions) (Follicular vs. Males: $p=0.1924$; Luteal vs. Males: $\mathrm{p}=0.6107)$. Serum BDNF levels (averaged over both sessions) were not correlated with fitness level for either sex (Pearson's: Females: $r=-0.029, \mathrm{p}=$ 0.934; Males: $r=-0.217, p=0.476$ ) or with progesterone (Spearman's: $r=0.227, p=0.266$ ) or estradiol in females (Spearman's: $r=0.228, \mathrm{p}=0.262$ ). 
Exercise details are presented in Table 3. All participants performed the exercise intervention at $\sim 68 \%$ of their maximal heart rate during both sessions $(\mathrm{p}=0.428)$. RPE and $\mathrm{EMG}_{\text {exercise }}$ were similar across all sessions for both sexes $(\mathrm{p}=0.428$ and $\mathrm{p}=0.290$, respectively). Cortisol levels decreased following exercise (paired t-test: $\mathrm{p}<0.01$ ).

\section{Motor Thresholds and M-Max}

For RMT, three-way ANOVA revealed a TIME*SEX interaction $\left(\mathrm{F}_{(1,26)}=7.099, \mathrm{p}=0.013\right)$ such that males had lower RMT at T0 compared to females (Tukey's: $p<0.0001$ ). Further, RMT decreased in males following exercise (Tukey's: $\mathrm{p}=0.015)$, although the effect was small $(\mathrm{g}=$ 0.26). RMT was unchanged following exercise in females (Tukey's: $p=0.566$ ). For AMT, threeway ANOVA revealed a main effect of $\operatorname{TIME}\left(\mathrm{F}_{(1,25)}=5.465, \mathrm{p}=0.028\right)$ such that AMT decreased following exercise (paired t-test: $\mathrm{p}=0.024, \mathrm{~g}=0.24$ ). Due to the small effect sizes, it is more appropriate to conclude that the change in RMT and AMT following exercise was negligible (Sullivan and Feinn, 2012). A three-way ANOVA on M-Max revealed no significant main effects or interactions (Table 4).

\section{MEP Recruitment Curves}

Group-averaged MEP recruitment curves normalized to M-Max (with standard error) for females are shown in Figure 3A. The shaded area indicates $25 \%$ above and below the point of inflection, from which AURC was obtained $(120-147 \%$ RMT). Two-way ANOVA revealed a main effect of TIME $\left(\mathrm{F}_{(1,13)}=6.851, \mathrm{p}=0.021\right)$ such that corticospinal excitability was greater at $\mathrm{T} 1$ compared to T0 (Wilcoxon signed-rank: $\mathrm{p}<0.001$; Figure 3B, right). Individual data are shown in Figure 3C 
M.Sc. Thesis - J. El-Sayes; McMaster University - Kinesiology

and indicate an increase AURC in 10 (asterisks) females, a decrease in 3, and no change in 1 female. The relationship between change in corticospinal excitability and BDNF is plotted in Figure 3D and shows that serum BDNF levels were correlated with percent change in corticospinal excitability (Pearson's: $r=0.559, p=0.047$ ), such that higher serum BDNF levels were associated with a greater percent change in AURC. Percent change in AURC was not correlated with fitness level (Pearson's: $\mathrm{r}=-0.109, \mathrm{p}=0.710$ ) or percent change in cortisol (Pearson's: $\mathrm{r}=-0.179, \mathrm{p}=$ $0.540)$.

Group-averaged MEP recruitment curves normalized to M-Max (with standard error) for males are shown in Figure 4A. The shaded area indicates $25 \%$ above and below the point of inflection, from which AURC was obtained (118 - 144\% RMT). Two-way ANOVA revealed no main effects or interactions (Table 5; Figure 4B). AURC was then averaged across the two sessions and revealed no difference between T0 and T1 (Wilcoxon signed-rank: $\mathrm{p}=0.198$ ). Individual data are shown in Figure 4C and show variable responses following exercise (increase in 5 males, a decrease in 2, no change in 7). Percent change in AURC was not correlated with serum BDNF (Figure 4D; Pearson's: $r=-0.108, p=0.725)$, fitness level (Pearson's: $r=-0.220, p=0.450$ ), or percent change in cortisol (Pearson's: $r=0.372, \mathrm{p}=0.190)$.

\section{Short Interval Intracortical Inhibition}

Unconditioned MEPs (MEP ${ }_{\mathrm{TS}}$ ) were maintained at $\sim 1 \mathrm{mV}$ for SICI at each CS intensity (Table 5, TS $-\mathrm{SICI}_{80}$ and TS $-\mathrm{SICI}_{90}$ ). The depth of SICI at $90 \%$ AMT was greater than that at $80 \%$ AMT $\left(\mathrm{F}_{(1,23)}=9.340, \mathrm{p}=0.006\right)$, and so $\mathrm{SICI}_{80}$ and $\mathrm{SICI}_{90}$ were analyzed separately. In females, a two- 
way ANOVA revealed no main effects or interactions for $\mathrm{SICI}_{80}$ or $\mathrm{SICI}_{90}$ (Figures $5 \mathrm{Ai}$ and 4Aii, respectively). Individual data are plotted in Figure 5B. SICI 80 (Figure 5B left) was reduced in 7 (asterisks), increased in 3, and did not change in 2 females. SICI $_{90}$ (Figure 5B right) was reduced in 5 (asterisks), increased in 3, and did not change in 3 females. In males, two-way ANOVAs revealed no main effects or interactions for $\mathrm{SICI}_{80}$ or SICI90 (Figures 6Ai and 5Aii, respectively). Individual data are plotted in Figure 6B. SICI 80 (Figure 6B left) was reduced in 8 (asterisks), increased in 3 , and did not change in 3 males. SICI 90 (Figure 6B right) was reduced in 8 (asterisks), increased in 4, and did not change in 2 males.

\section{Discussion}

The present study examined the effects of biological sex and ovarian hormones on neuroplasticity induced by a single session of exercise. We report novel findings indicating that only females demonstrated a significant increase in corticospinal excitability following exercise, and these changes were related to baseline levels of serum BDNF. Further, this increase was observed in both the follicular and luteal phases of the menstrual cycle. Males did not show a significant increase in corticospinal excitability following exercise. SICI was not changed by exercise in either sex. We discuss these findings and their implications below.

\section{Increases in Corticospinal Excitability in Females}

In this study, we observed a significant increase in corticospinal excitability in females ( $33 \%$ increase in AURC), but not males ( $15 \%$ increase in AURC). Observations of greater acute neuroplasticity in females are reported elsewhere (see review Ridding and Ziemann, 2010), but not consistently (Gorbet and Staines, 2011). Previous neurophysiology research shows a similar 
M.Sc. Thesis - J. El-Sayes; McMaster University - Kinesiology

trend, such that cathodal transcranial direct current stimulation (tDCS) reduces MEPs by $\sim 20 \%$ in females but not in males (Kuo et al., 2006). Further, anodal tDCS increases visual evoked potentials in females but not males (Chaieb et al., 2008).

The explanation for the difference in MEP response between sexes is unclear, however we suggest three potential factors may contribute. First, peripheral BDNF protein increases following exercise (see review Huang et al., 2014) and is thought to mediate exercise-induced neuroplasticity (Cotman et al., 2007). BDNF augments the amplitude and frequency of excitatory post-synaptic currents (Carmignoto et al., 1997; Lessmann et al., 1994; Levine et al., 1995). The differential effects of exercise on BDNF between sexes has not been directly investigated. However, exerciseinduced increases in peripheral BDNF have been observed in both males and females (see review Huang et al., 2014). Here, we show that greater levels of serum BDNF in females are associated with greater percent change in AURC (from T0 to T1). However, despite no differences in serum BDNF between sexes, males did not show this association. The relationship between BDNF levels and excitability may be due to females having higher brain levels of BDNF expression compared to males (Michalski et al., 2015). As such, BDNF may be a potential mediator of acute exerciseinduced increases in corticospinal excitability in females only.

Second, BDNF promotes differentiation of serotonergic and dopaminergic neurons and increases serotonin and dopamine release (Altar et al., 1992; Martinowich and Lu, 2008; Studer et al., 1996). As such, greater BDNF levels in females may promote increased serotonin and dopamine concentration in females compared to males (Laakso et al., 2002; Ortiz et al., 1988; Weiss et al., 
M.Sc. Thesis - J. El-Sayes; McMaster University - Kinesiology

2005). These neurotransmitter levels increase following acute exercise (Gomez-Merino et al., 2001; Kitaoka et al., 2010; Meeusen et al., 1997), and may mediate increases in MEPs. Serotonin promotes facilitation in the motor cortex, as the effects of excitatory paired associated stimulation (PAS) are enhanced by the administration of a serotonin reuptake inhibitor (Batsikadze et al., 2013). Further, serotonin receptor activity facilitates activation of tropomyosin-related kinase B, the BDNF receptor, and increases BDNF mRNA transcription (Homberg et al., 2014; Martinowich and Lu, 2008). Dopamine promotes motor cortex plasticity (Rioult-Pedotti et al., 2015), and an increase in D2 receptor activity elevates spike firing rate in pyramidal neurons (Vitrac et al., 2014). Dopamine and BDNF also share a common pathway (i.e. activating phospholipase C), thereby increasing excitability through similar mechanisms (Vitrac and Benoit-Marand, 2017). Therefore, although males may also show increased serotonin and dopamine following exercise, this increase may not be sufficient to result in an increase in MEPs due to lower baseline levels of serotonin and dopamine.

Third, estradiol reduces the firing threshold of neurons and enhances glutamatergic neurotransmission (Finocchi and Ferrari, 2011; Grassi et al., 2010). Further, estradiol reduces the activity of $\mathrm{GABA}_{\mathrm{A}}$ receptors, thereby increasing the excitability of pyramidal neurons (Murphy et al., 1998). This increase in pyramidal neuron excitability also results in increased levels of activitydependent BDNF (Scharfman and MacLusky, 2006, 2005). BDNF expression is also enhanced by estradiol, as the BDNF gene contains an estrogen-like response element (Scharfman and MacLusky, 2006, 2005). Although we did not show a correlation between estradiol and serum BDNF, estradiol and plasma BDNF levels are positively correlated (Begliuomini et al., 2007). Last, estrogen and BDNF facilitate excitability through common signaling pathways (Scharfman 
and MacLusky, 2006). Therefore, the presence of high estradiol levels in females may allow for BDNF-induced increases in MEPs post-exercise.

We exclude cortisol as a mediator of the increase in MEPs observed following exercise. Cortisol enhances GABAergic neurotransmission (Di et al., 2009), reduces glutamatergic neurotransmission (Di et al., 2009), and abolishes MEP facilitation following excitatory paired associative stimulation (Sale et al., 2008). We observed a reduction in cortisol following exercise, as seen elsewhere (Heaney et al., 2013; Ida et al., 2013; Park et al., 2018). However, despite a reduction in cortisol in both sexes, we only observed an increase in MEPs in females. Therefore, it is unlikely that exercise-induced reductions in cortisol mediated the increases in MEPs. Further, the change in corticospinal excitability is not associated with the change in cortisol in either sex, suggesting that the increase in excitability observed in females is not mediated by reductions in cortisol. Last, it is important to note that the reductions in cortisol we observed may not be solely due to the exercise intervention. In the present and previous studies (Heaney et al., 2013; Park et al., 2018), participants were tested in the morning which is the time of greatest reduction in cortisol due to circadian rhythm (Pluchino et al., 2009).

\section{Sex Differences in RMT}

We observed greater RMT in females at baseline (i.e. pre-exercise) compared to males. Previous work has shown no difference in RMT between sexes (Livingston et al., 2010; Pitcher et al., 2003; Shibuya et al., 2016; Zoghi et al., 2015). This discrepancy may be due to our participant sampling. Our males and females were tightly age- and fitness-matched. Comparatively, other studies 
M.Sc. Thesis - J. El-Sayes; McMaster University - Kinesiology

recruited participants from a larger age range and did not assess or match for fitness level (Livingston et al., 2010; Pitcher et al., 2003; Shibuya et al., 2016; Zoghi et al., 2015). This lack of consistency between studies may explain our observation of RMT differences between sexes.

\section{Change in RMT and AMT}

Previous work has shown no change in RMT (Garnier et al., 2017; Lulic et al., 2017; Singh et al., 2014; Smith et al., 2014) or AMT (Lulic et al., 2017) following exercise. Although we observed reductions in RMT in males and in AMT in both sexes following exercise, the effect sizes were very small, suggesting that they are negligible (Sullivan and Feinn, 2012). Effect sizes are an important consideration, as they indicate the magnitude of difference between groups or timepoints (Sullivan and Feinn, 2012). The effect sizes for reductions in RMT and AMT were $g=0.26$ and 0.24, respectively, which is considered a small effect (Cohen, 1988). Further, RMT and AMT were reduced by $1 \%$ and $0.5 \%$ maximum stimulator output following exercise, respectively. Previous work has shown that the within-subject variability of motor thresholds is $8.5 \%$ of motor threshold (Tranulis et al., 2006), indicating that the exercise likely did not significantly reduce RMT and AMT. Rather, the change we observe is simply due to variability during data acquisition.

\section{No Exercise-Induced Change in SICI}

SICI did not change following aerobic exercise, similar to results reported elsewhere (Mooney et al., 2016) but dissimilar to the reduction in SICI frequently shown (Lulic et al., 2017; Singh et al., 2014; Smith et al., 2014; Stavrinos and Coxon, 2017). Discrepancies between studies may result from the different fitness levels of the participant cohorts. The present study and Mooney et al. 
M.Sc. Thesis - J. El-Sayes; McMaster University - Kinesiology

(2016) recruited recreationally active individuals, assessed via $\mathrm{VO}_{2}$ peak, and both studies demonstrated no changes in SICI. Comparatively, other studies (Singh et al., 2014; Smith et al., 2014; Stavrinos and Coxon, 2017) tested low to moderately active individuals (defined by IPAQ) and demonstrated reductions in SICI. Therefore, acute exercise does not appear to modulate SICI in individuals with higher cardiorespiratory fitness. Individuals with higher cardiorespiratory fitness may have differences in GABA concentrations or GABA receptor function compared to individuals with lower cardiorespiratory fitness, but this remains to be investigated.

\section{Limitations \& Future Considerations}

In females, we observed an increase in the neuronal output to a hand muscle following exercise, the goal of some rehabilitation approaches. Comparatively, males did not show this increase. In this study, BDNF was not assessed post-exercise, limiting our ability to determine the role of BDNF in mediating the exercise-induced changes observed here. We did not assess BDNF postexercise due to the high variability of the measure. However, future research may benefit from relating the exercise-induced changes in BDNF to changes in TMS measures. Further, although it has been suggested that peripheral BDNF levels may reflect brain BDNF levels (Krabbe et al., 2007), this remains unclear. BDNF is synthesized in both in the periphery (Wang et al., 2006) and in the central nervous system (Fujimura et al., 2002; Pan et al., 1998), but can only be obtained in vivo peripherally. BDNF is stored in platelets (Fujimura et al., 2002), and the analysis of BDNF levels is impacted by the completeness of blood clotting and the plasma isolation protocol (Fujimura et al., 2002). Further, ELISA kits used to analyze BDNF levels are susceptible to artifacts due to low specificity of BDNF antibodies and the presence of BDNF binding protein in the blood (Skornicka et al., 2002). Therefore, due to these confounding factors it remains unclear 
if obtained peripheral BDNF levels are a true reflection of brain BDNF. This work only investigated females at two points of the menstrual cycle (mid-follicular and mid-luteal). Therefore, it remains unclear if acute exercise will induce comparable neuroplasticity at other points of the cycle (e.g. during menstruation when both estradiol and progesterone levels are low). Finally, it is important to note that these data were obtained in healthy, active, young adults and so is limited in generalizability to this population. Further work is needed to establish if these findings extend to aging or clinical populations.

\section{Conclusion}

The present study demonstrated that biological sex impacts the propensity for exercise-induced neuroplasticity. Corticospinal excitability was increased following exercise in females only, and this increase was positively associated with levels of serum BDNF. The present work also shows that changes in ovarian hormones across the menstrual cycle do not modulate exercise-induced neuroplasticity. Therefore, it is important to consider biological sex when investigating neuroplasticity induction following acute exercise. 
M.Sc. Thesis - J. El-Sayes; McMaster University - Kinesiology

\section{Tables and Figures}

Table 1: Descriptive Characteristics of Participants.

\begin{tabular}{|c|c|c|c|}
\hline & Male & Female & $\mathrm{p}$-value \\
\hline Age (years) & $21 \pm 2$ & $21 \pm 2$ & $\mathrm{p}=0.412^{*}$ \\
\hline Height $(\mathrm{cm})$ & $178 \pm 7$ & $163 \pm 7$ & $\mathbf{p}=\mathbf{0 . 0 0 0}$ \\
\hline Weight (kg) & $70 \pm 8$ & $59 \pm 7$ & $p=0.010$ \\
\hline FFM $(\mathrm{kg})$ & $63 \pm 6$ & $44 \pm 5$ & $\mathbf{p}=\mathbf{0 . 0 0 0}$ \\
\hline Body fat $(\%)$ & $11 \pm 5$ & $26 \pm 5$ & $\mathbf{p}=\mathbf{0 . 0 0 0}$ \\
\hline $\mathrm{VO}_{2}$ peak $(\mathrm{ml} / \mathrm{kg} / \mathrm{min})$ & $50 \pm 6$ & $43 \pm 6$ & $p=0.046$ \\
\hline $\mathrm{VO}_{2}$ peak $(\mathrm{ml} / \mathrm{kgFFM} / \mathrm{min})$ & $56 \pm 7$ & $58 \pm 7$ & $\mathrm{p}=0.395$ \\
\hline
\end{tabular}


M.Sc. Thesis - J. El-Sayes; McMaster University - Kinesiology

Table 2: Hormones and Brain-Derived Neurotrophic Factor Levels Across Sessions in Both Sexes

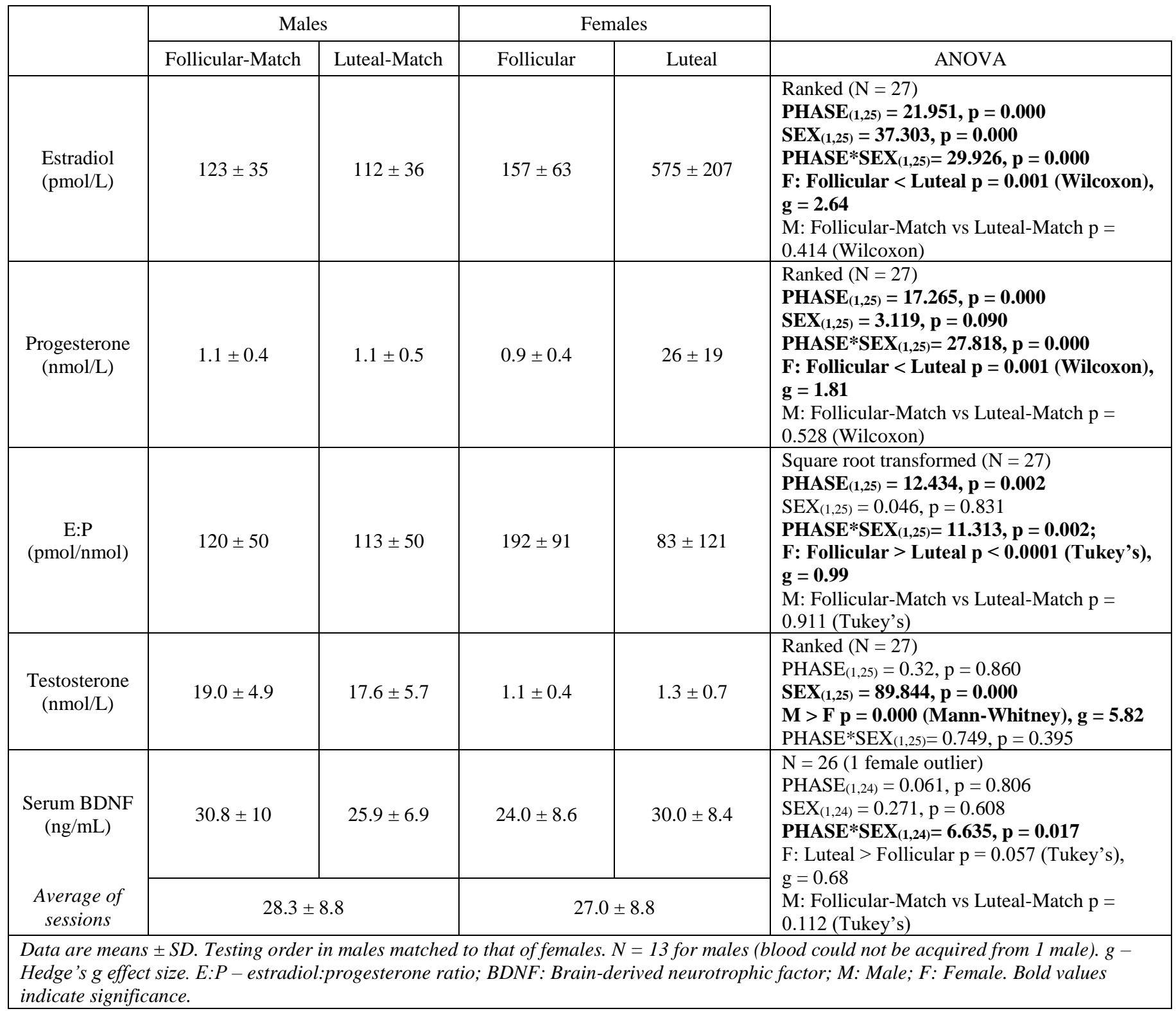


M.Sc. Thesis - J. El-Sayes; McMaster University - Kinesiology

Table 3: Exercise Details Across Sessions in Both Sexes

\begin{tabular}{|c|c|c|c|c|c|c|}
\hline & & \multicolumn{2}{|c|}{ Males } & \multicolumn{2}{|c|}{ Females } & \multirow[b]{2}{*}{ ANOVA } \\
\hline & & $\begin{array}{l}\text { Follicular- } \\
\text { Match }\end{array}$ & $\begin{array}{l}\text { Luteal- } \\
\text { Match }\end{array}$ & Follicular & Luteal & \\
\hline \multicolumn{2}{|c|}{$\begin{array}{l}\text { Heart rate } \\
\text { (bpm) }\end{array}$} & $126 \pm 8$ & $127 \pm 8$ & $129 \pm 11$ & $130 \pm 11$ & $\begin{array}{l}\text { Ranked }(\mathrm{N}=28) \\
\text { PHASE }_{(1,26)}=1.322, \mathrm{p}=0.261 \\
\text { SEX }_{(1,26)}=1.006, p=0.325 \\
\text { PHASE*SEX }_{(1,26)}=0.022, \mathrm{p}=0.883\end{array}$ \\
\hline \multicolumn{2}{|c|}{$\% \mathrm{HR}_{\max }$} & $67.3 \pm 3.0$ & $67.7 \pm 2.1$ & $68.4 \pm 4.0$ & $68.9 \pm 3.2$ & $\begin{array}{l}\mathrm{N}=28 \\
\operatorname{PHASE}_{(1,26)}=0.917, \mathrm{p}=0.347 \\
\text { SEX }_{(1,26)}=0974, p=0.333 \\
\text { PHASE*SEX }_{(1,26)}=0.020, \mathrm{p}=0.889\end{array}$ \\
\hline \multicolumn{2}{|c|}{$\begin{array}{l}\text { RPE } \\
(0-10)\end{array}$} & $2.7 \pm 0.9$ & $3.0 \pm 0.9$ & $2.5 \pm 0.7$ & $2.6 \pm 0.7$ & $\begin{array}{l}\mathrm{N}=28 \\
\operatorname{PHASE}_{(1,26)}=1.013, \mathrm{p}=0.323 \\
\operatorname{SEX}_{(1,26)}=1.325, \mathrm{p}=0.260 \\
\text { PHASE}^{*} \operatorname{SEX}_{(1,26)}=0.650, \mathrm{p}=0.428\end{array}$ \\
\hline \multicolumn{2}{|c|}{$\mathrm{EMG}_{\text {exercise }}$} & $73.0 \pm 11.7$ & $63.7 \pm 29.0$ & $68.9 \pm 8.7$ & $73.2 \pm 3.1$ & $\begin{array}{l}\text { Ranked }(\mathrm{N}=27) \\
\text { PHASE }_{(1,25)}=0.177, \mathrm{p}=0.677 \\
\text { SEX }_{(1,25)}=0.054, \mathrm{p}=0.818 \\
\text { PHASE*SEX }_{(1,25)}=1.168, \mathrm{p}=0.290\end{array}$ \\
\hline \multirow{2}{*}{$\begin{array}{c}\text { Cortisol } \\
\text { (nmol/L) }\end{array}$} & T0 & $5.0 \pm 2.9$ & $4.5 \pm 2.8$ & $4.0 \pm 3.4$ & $2.5 \pm 1.7$ & \multirow{2}{*}{ 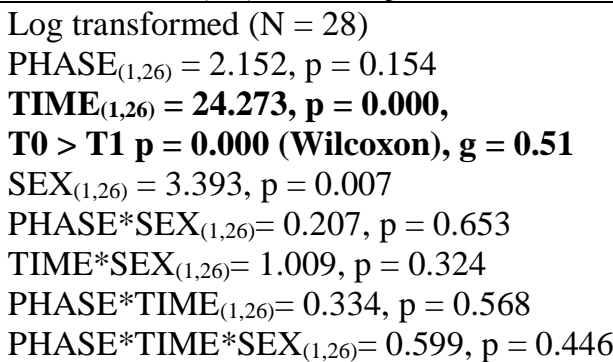 } \\
\hline & $\mathrm{T} 1$ & $4.2 \pm 2.5$ & $3.6 \pm 2.2$ & $3.5 \pm 3.7$ & $2.2 \pm 1.8$ & \\
\hline \multicolumn{7}{|c|}{$\begin{array}{l}\text { Data are means } \pm S D . \text { Testing order in males matched to that of females. } g-H e d g e ' s g \text { effect size. } \% H R_{\text {max: }} \text { percent of } \\
\text { maximum heart rate; RPE: ratings of perceived exertion. EMG } G_{\text {exercise: }} \text { level of EMG activity from FDI during exercise; TO: } \\
\text { pre-exercise; T1: post-exercise. Bold values indicate significance. }\end{array}$} \\
\hline
\end{tabular}


M.Sc. Thesis - J. El-Sayes; McMaster University - Kinesiology

Table 4: RMT, AMT, and M-Max Across Sessions in Both Sexes

\begin{tabular}{|c|c|c|c|c|c|c|c|c|c|}
\hline & \multicolumn{4}{|c|}{ Males } & \multicolumn{4}{|c|}{ Females } & \multirow{3}{*}{ ANOVA } \\
\hline & \multicolumn{2}{|c|}{ Follicular-Match } & \multicolumn{2}{|c|}{ Luteal-Match } & \multicolumn{2}{|c|}{ Follicular } & \multicolumn{2}{|c|}{ Luteal } & \\
\hline & T0 & $\mathrm{T} 1$ & T0 & $\mathrm{T} 1$ & T0 & $\mathrm{T} 1$ & T0 & $\mathrm{T} 1$ & \\
\hline RMT\# & $\begin{array}{c}37.4 \pm \\
5.0\end{array}$ & $\begin{array}{c}35.8 \pm \\
4.6\end{array}$ & $\begin{array}{c}37.4 \pm \\
5.4\end{array}$ & $\begin{array}{c}36.3 \pm \\
4.9\end{array}$ & $\begin{array}{c}40.1 \pm \\
6.9\end{array}$ & $\begin{array}{c}40.4 \pm \\
6.5\end{array}$ & $\begin{array}{c}40.7 \pm \\
7.7\end{array}$ & $\begin{array}{c}41.1 \pm \\
8.3\end{array}$ & 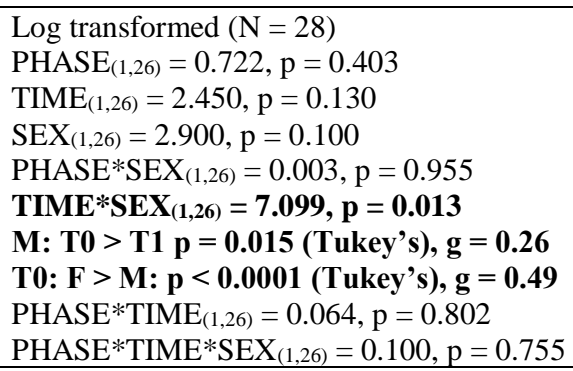 \\
\hline $\mathrm{AMT}$ & $\begin{array}{c}27.9 \pm \\
3.0\end{array}$ & $\begin{array}{c}26.9 \pm \\
3.2\end{array}$ & $\begin{array}{c}25.9 \pm \\
7.1\end{array}$ & $\begin{array}{c}25.1 \pm \\
6.9\end{array}$ & $\begin{array}{c}29.9 \pm \\
4.4\end{array}$ & $\begin{array}{c}29.5 \pm \\
6.2\end{array}$ & $\begin{array}{c}29.6 \pm \\
4.2\end{array}$ & $\begin{array}{c}29.6 \pm \\
5.3\end{array}$ & 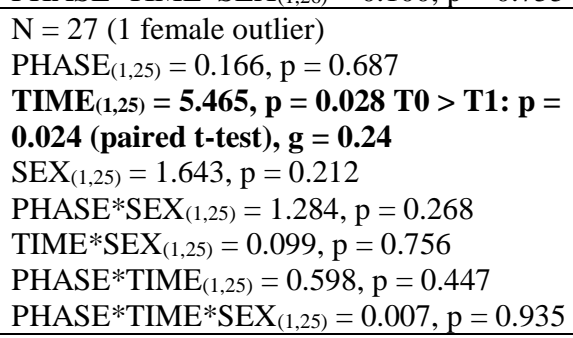 \\
\hline M-Max* & $\begin{array}{c}11.9 \pm \\
4.7\end{array}$ & $\begin{array}{c}10.6 \pm \\
4.6\end{array}$ & $\begin{array}{c}11.6 \pm \\
4.7\end{array}$ & $\begin{array}{c}10.8 \pm \\
5.0\end{array}$ & $\begin{array}{c}12.5 \pm \\
3.1\end{array}$ & $\begin{array}{c}12.2 \pm \\
3.4\end{array}$ & $\begin{array}{c}13.1 \pm \\
3.6\end{array}$ & $\begin{array}{c}12.4 \pm \\
3.7\end{array}$ & $\begin{array}{l}\text { Ranked }(\mathrm{N}=28) \\
\operatorname{PHASE}_{(1,26)}=1.790, \mathrm{p}=0.192 \\
\operatorname{TIME}_{(1,26)}=2.853, \mathrm{p}=0.103 \\
\operatorname{SEX}_{(1,26)}=0.513, \mathrm{p}=0.480 \\
\operatorname{PHASE}^{*} \operatorname{SEX}_{(1,26)}=0.573, \mathrm{p}=0.456 \\
\text { TIME*SEX }_{(1,26)}=0.051, \mathrm{p}=0.822 \\
\text { PHASE}^{*} \operatorname{TIME}_{(1,26)}=0.176, \mathrm{p}=0.678 \\
\text { PHASE}^{*} \operatorname{TIME}^{*} \operatorname{SEX}_{(1,26)}=2.057, \mathrm{p}=0.163\end{array}$ \\
\hline
\end{tabular}


Table 5: Statistical Outcomes of AURC and SICI (each normalized to M-Max)

\begin{tabular}{|c|c|c|}
\hline \multirow{2}{*}{$\begin{array}{l}\text { Dependent } \\
\text { Measure }\end{array}$} & \multicolumn{2}{|c|}{ ANOVA } \\
\hline & Females & Males \\
\hline AURC & $\begin{array}{l}\text { Log transformed }(\mathrm{N}=14) \\
\operatorname{PHASE}_{(1,13)}=0.061, \mathrm{p}=0.383 \\
\operatorname{TIME}_{(1,13)}=\mathbf{6 . 8 5 1}, \mathbf{p}=\mathbf{0 . 0 2 1} \\
\operatorname{PHASE} \mathrm{TIME}_{(1,13)}=1.696, \mathrm{p}=0.215 \\
\text { T1> T0: } \mathbf{p}=\mathbf{0 . 0 0 9}(\text { Wilcoxon), } \mathbf{g}=\mathbf{0 . 4 2} \\
\text { T0: } \mathbf{2 . 2 3} \pm \mathbf{1 . 1 9} \text { T1: } \mathbf{3 . 0 4} \pm \mathbf{2 . 3 5}(\text { averaged over } \\
\text { phase) }\end{array}$ & $\begin{array}{l}\text { Log transformed }(\mathrm{N}=14) \\
\operatorname{PHASE}_{(1,13)}=0.581, \mathrm{p}=0.459 \\
\operatorname{TIME}_{(1,13)}=0.141, \mathrm{p}=0.713 \\
\text { PHASE*TIME }_{(1,13)}=0.316, \mathrm{p}=0.583 \\
\text { T1> T0: } \mathrm{p}=0.198 \text { (Wilcoxon) } \\
\text { T0: } 2.97 \pm 1.68 \mathrm{~T} 1: 3.38 \pm 2.00 \text { (averaged over } \\
\text { sessions) }\end{array}$ \\
\hline $\mathrm{TS}-\mathrm{SICI}_{80}$ & $\begin{array}{l}\mathrm{N}=12, \text { technical error in acquisition from } 2 \\
\text { females } \\
\operatorname{PHASE}_{(1,11)}=1.072, \mathrm{p}=0.323 \\
\text { TIME }_{(1,11)}=1.212, \mathrm{p}=0.294 \\
\text { PHASE*TIME }_{(1,11)}=0.418, \mathrm{p}=0.531\end{array}$ & $\begin{array}{l}\mathrm{N}=14 \\
\operatorname{PHASE}_{(1,13)}=0.137, \mathrm{p}=0.718 \\
\operatorname{TIME}_{(1,13)}=1.299, \mathrm{p}=0.275 \\
\operatorname{PHASE}^{*} \operatorname{TIME}_{(1,13)}=0.040, \mathrm{p}=0.845\end{array}$ \\
\hline $\mathrm{TS}-\mathrm{SICI}_{90}$ & $\begin{array}{l}\mathrm{N}=11 \text {, technical error in acquisition from } 3 \\
\text { females } \\
\text { PHASE }_{(1,10)}=0.027, p=0.872 \\
\text { TIME }_{(1,10)}=0.003, p=0.958 \\
\text { PHASE*TIME }_{(1,10)}=1.945, p=0.193\end{array}$ & $\begin{array}{l}\mathrm{N}=14 \\
\operatorname{PHASE}_{(1,13)}=1.501, \mathrm{p}=0.242 \\
\operatorname{TIME}_{(1,13)}=0.022, \mathrm{p}=0.885 \\
\operatorname{PHASE} \operatorname{TIME}_{(1,13)}=0.001, \mathrm{p}=0.980\end{array}$ \\
\hline $\mathrm{SICI}_{80}$ & $\begin{array}{l}\mathrm{N}=12 \text {, technical error in acquisition from } 2 \\
\text { females } \\
\operatorname{PHASE}_{(1,11)}=0.200, p=0.633 \\
\text { TIME }_{(1,11)}=0.001, p=0.084 \\
\text { PHASE } \operatorname{TIME}_{(1,11)}=0.119, \mathrm{p}=0.736 \\
\text { T0 vs T1: } \mathrm{p}=0.084 \text { (paired t-test) } \\
\text { T0: } 0.07 \pm 0.03 \mathrm{~T} 1: 0.08 \pm 0.02 \text { (averaged over } \\
\text { phase) }\end{array}$ & $\begin{array}{l}\text { Square root transformed }(\mathrm{N}=14) \\
\text { PHASE }_{(1,13)}=1.324, p=0.271 \\
\text { TIME }_{(1,13)}=1.127, p=0.308 \\
\text { PHASE*TIME } \\
\\
\text { T0 vs T1: } 1 \text { p }=0.177 \text { (Wilcoxon) } \\
\text { T0: } 0.08 \pm 0.05 \text { T1: } 0.09 \pm 0.05 \text { (averaged over } \\
\text { sessions) }\end{array}$ \\
\hline $\mathrm{SICI}_{90}$ & $\begin{array}{l}\mathrm{N}=11 \text {, technical error in acquisition from } 3 \\
\text { females } \\
\operatorname{PHASE}_{(1,10)}=0.010, p=0.922 \\
\operatorname{TIME}_{(1,10)}=0.681, p=0.428 \\
\operatorname{PHASE}^{*} \operatorname{TIME}_{(1,10)}=0.041, \mathrm{p}=0.844 \\
\text { T0 vs T1: } \mathrm{p}=0.428 \text { (paired t-test) } \\
\text { T0: } 0.06 \pm 0.02 \mathrm{~T} 1: 0.07 \pm 0.02 \text { (averaged over } \\
\text { phase) }\end{array}$ & $\begin{array}{l}\text { Log transformed }(\mathrm{N}=14) \\
\operatorname{PHASE}_{(1,13)}=1.268, \mathrm{p}=0.281 \\
\operatorname{TIME}_{(1,13)}=3.181, \mathrm{p}=0.098 \\
\text { PHASE*TIME }_{(1,13)}=0.089, \mathrm{p}=0.770 \\
\text { T0 vs T1: } \mathrm{p}=0.158 \text { (Wilcoxon) } \\
\text { T0: } 0.06 \pm 0.03 \text { T1: } 0.08 \pm 0.04 \text { (averaged over } \\
\text { sessions) }\end{array}$ \\
\hline rise; & lues indicate significance. & $\begin{array}{l}\text { dge's g effect siz } \\
\text { Inhibition; T0: }\end{array}$ \\
\hline
\end{tabular}


Figure 1

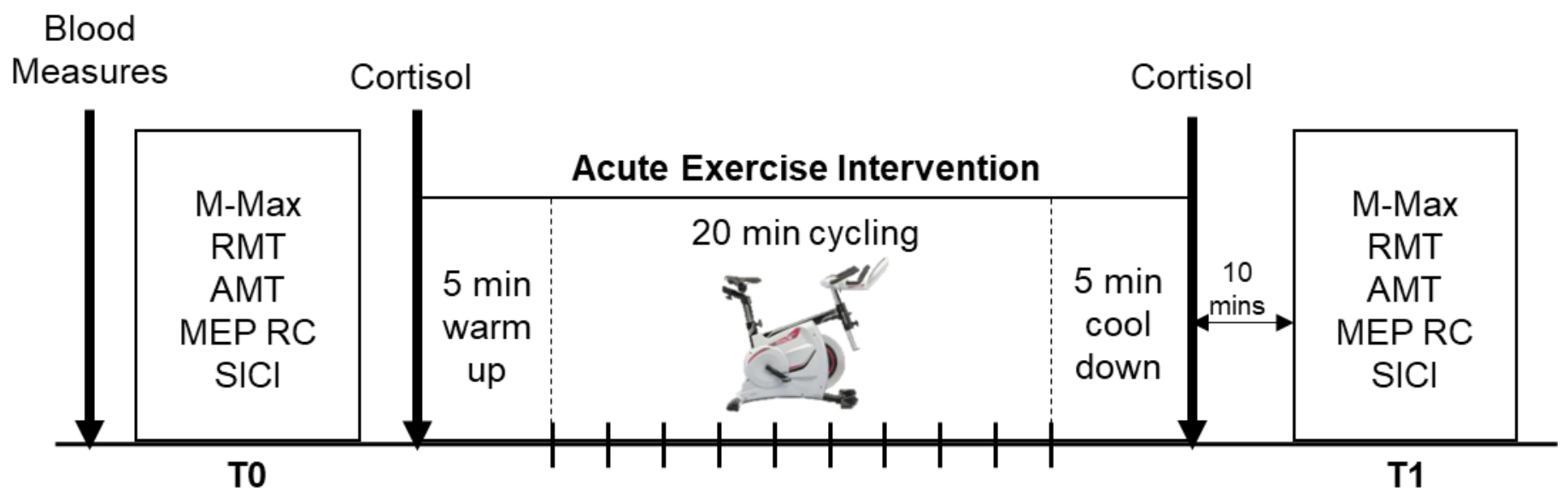

Figure 1: Experimental Timeline. Sessions 2 and 3 were identical. Blood measures were obtained at T0 to determine levels of sex hormones and brain-derived neurotrophic factor. Dependent measures were acquired before (T0) and 10 minutes following the exercise intervention (T1). Dependent measures included maximum M wave (M-Max), resting motor threshold (RMT), active motor threshold (AMT), motor evoked potential (MEP) recruitment curves (RC), and short interval intracortical inhibition (SICI). The acute exercise protocol was 30 minutes in total, including a 5-minute warm up and 5-minute cool down. During the 20-minute intervention, participants cycled at $65-70 \%$ of their maximum heart rate (i.e. moderate intensity). Rating of Perceived Exertion (RPE) was obtained every 2 minutes of the 20-minute intervention (indicated by tick marks). 
Figure 2

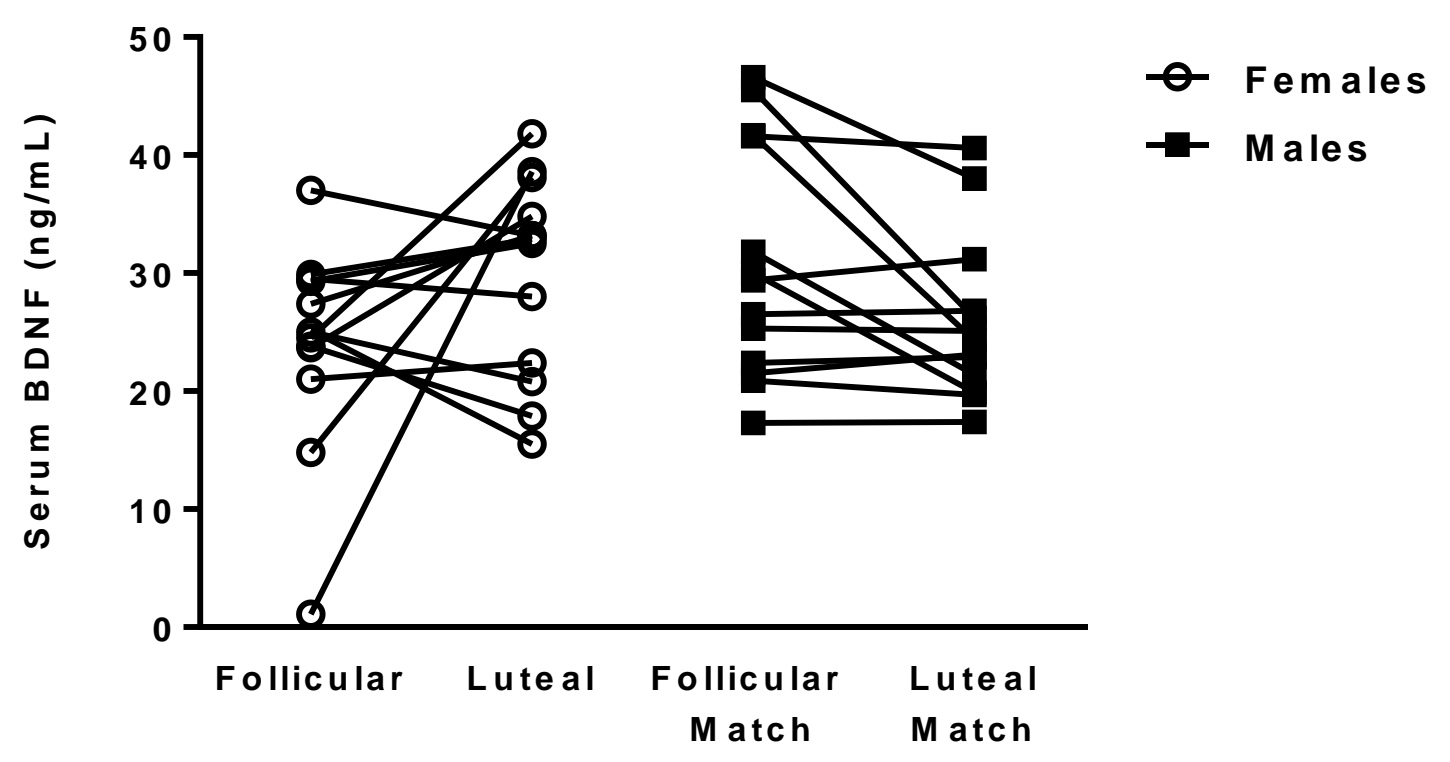

Figure 2: Serum BDNF Levels: Trend towards greater serum BDNF levels in the luteal phase compared to the follicular phase (Tukey's: $p=0.057$ ). BDNF levels are greater in the luteal phase in 6 females, with 4 females showing greater BDNF in the follicular phase and 2 females showing similar levels in both phases. Males did not show a significant difference between BDNF levels across sessions (Tukey's: $\mathrm{p}=0.112$ ). BDNF levels were similar in both sessions in 8 males, with 5 males showing greater BDNF levels in Follicular-Match compared to Luteal-Match. 
Figure 3
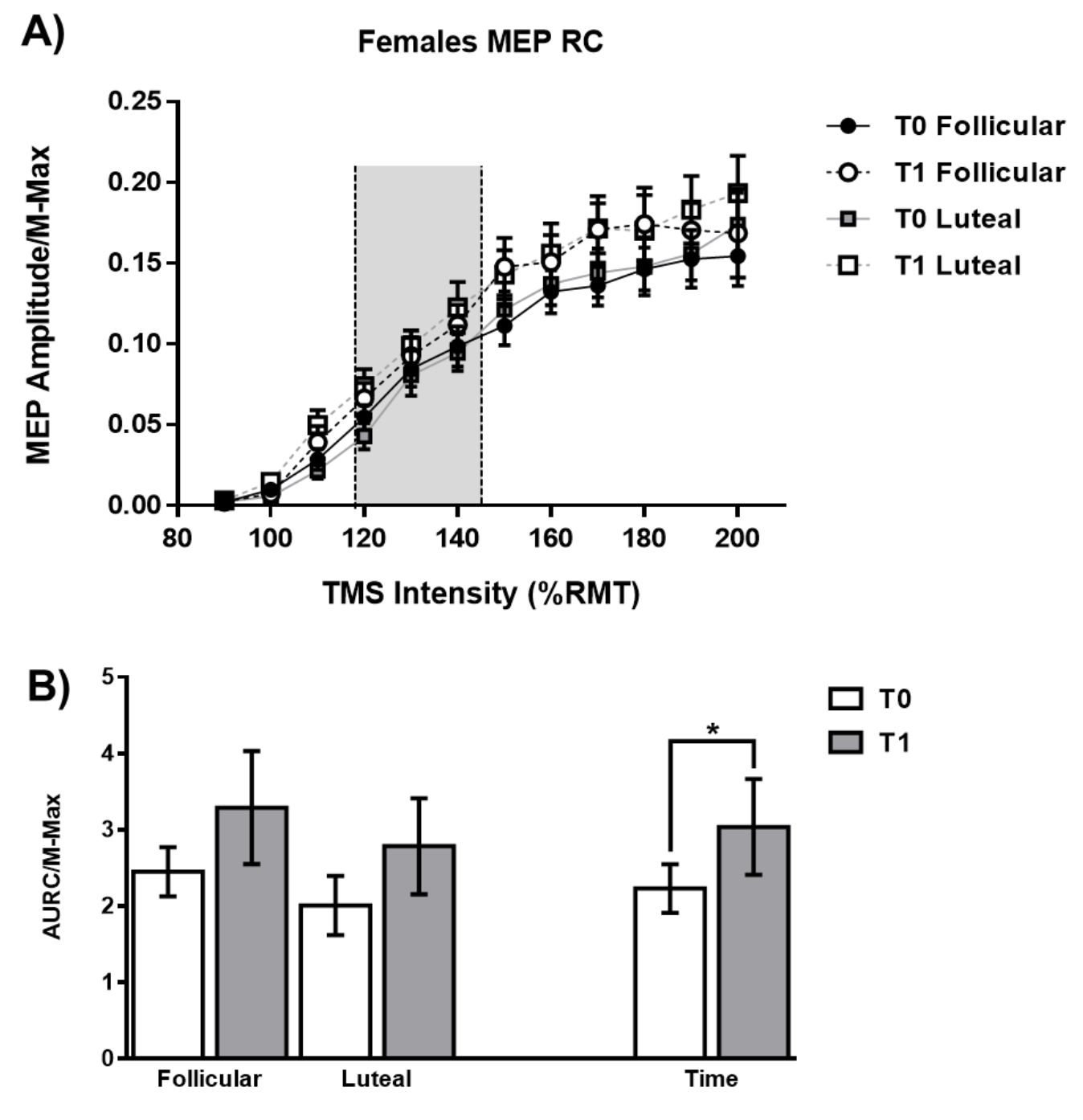

C)

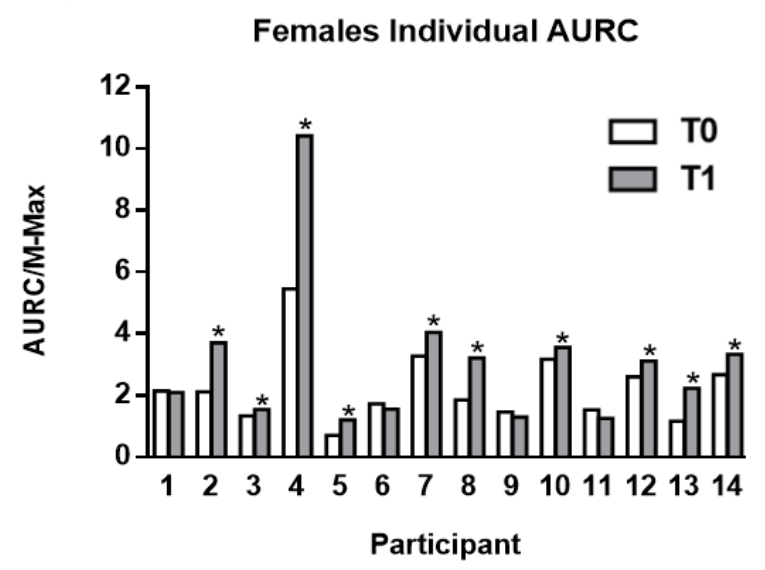

D)

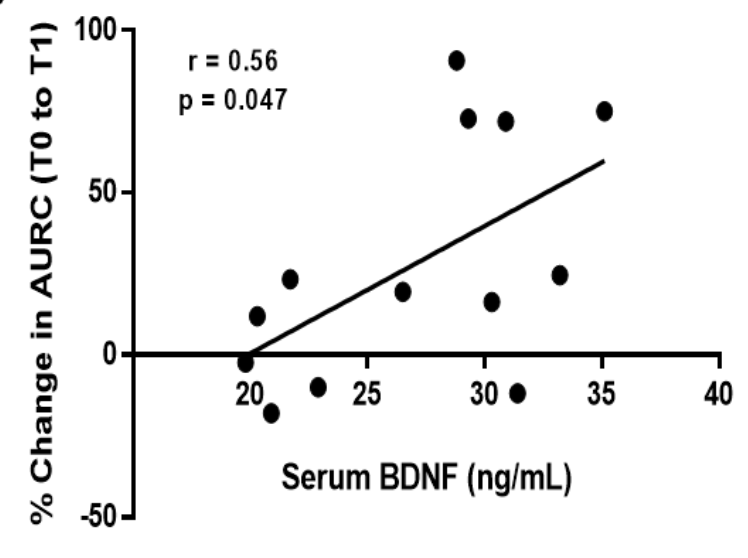


M.Sc. Thesis - J. El-Sayes; McMaster University - Kinesiology

Figure 3: MEP Recruitment Curves in Females. A) MEP recruitment curves show the MEP amplitude normalized to M-Max plotted against TMS intensity expressed as a percentage of RMT. Black lines indicate the follicular phase, grey lines indicate the luteal phase. Solid lines and filled symbols are pre-exercise (T0) and dashed lines and open symbols are post-exercise (T1). Shaded area indicates $25 \%$ above and below the point of inflection, from which AURC was obtained. B) AURC normalized to M-max increased post-exercise (T1), regardless of menstrual cycle phase. C) Individual data showing an increase in AURC in 10 females (asterisks), a decrease in 3, and no change in 1. D) Significant positive correlation between average serum BDNF and average percent change in AURC. T0 (pre-exercise): white bars; T1 (post-exercise): gray bars. Asterisks indicate a significance of $\mathrm{p}<0.05$ 
Figure 4
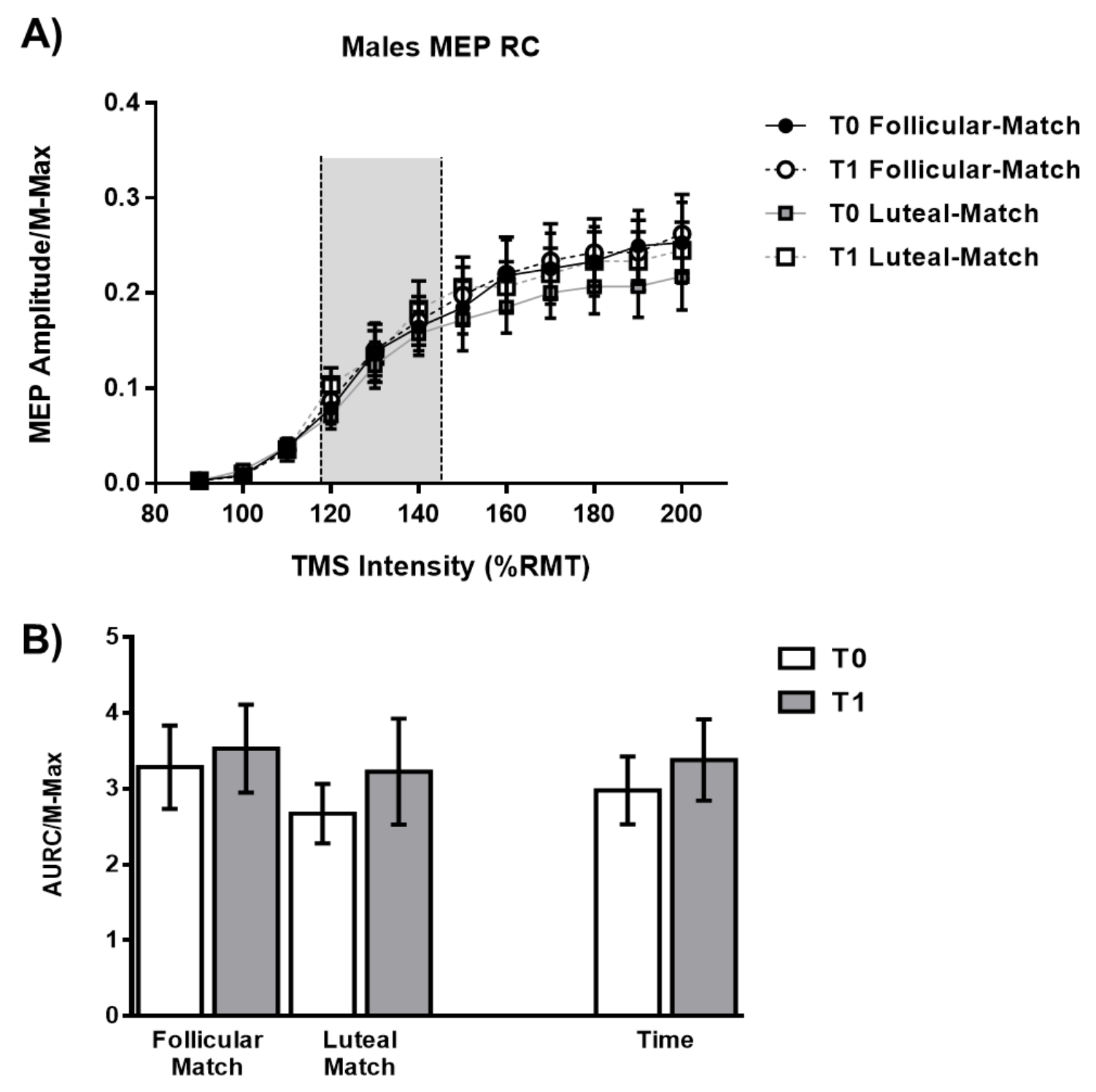

C)

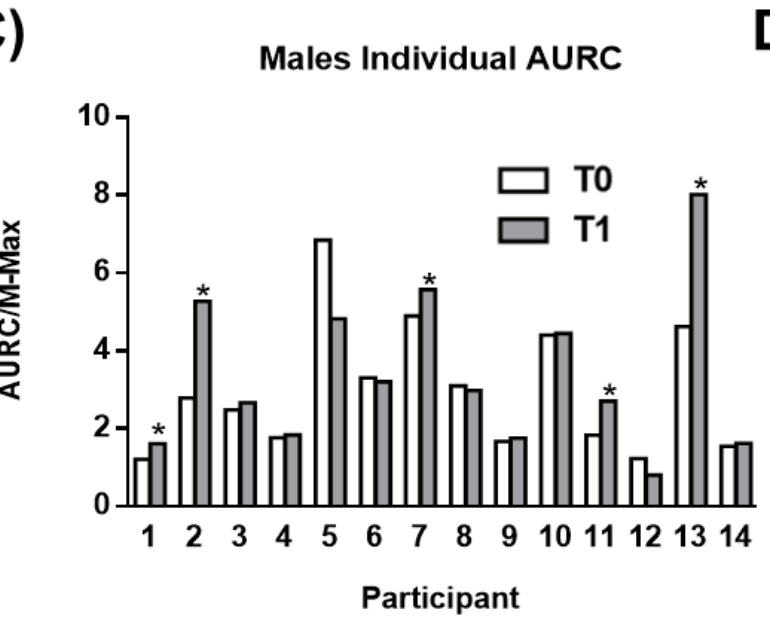

D)




M.Sc. Thesis - J. El-Sayes; McMaster University - Kinesiology

Figure 4: MEP Recruitment Curves in Males. Testing order in males was matched to that of females. A) MEP recruitment curves show the MEP amplitude normalized to M-Max plotted against TMS intensity expressed as a percentage of RMT. Black lines indicate Follicular-Match, grey lines indicate Luteal-Match. Solid lines and filled symbols are pre-exercise (T0) and dashed lines and open symbols are post-exercise (T1). Shaded area indicates $25 \%$ above and below the point of inflection, from which AURC was obtained. B) AURC normalized to M-max does not change post-exercise (T1). C) Individual data showing an increase in AURC in 5 males (asterisks), a decrease in 2, and no change in 7. D) No correlation between average serum BDNF and average percent change in AURC. T0 (pre-exercise): white bars; T1 (post-exercise): gray bars. Asterisks indicate a significance of $\mathrm{p}<0.05$ 
Figure 5

A)

Females

i) $\mathrm{SICl}_{80}$

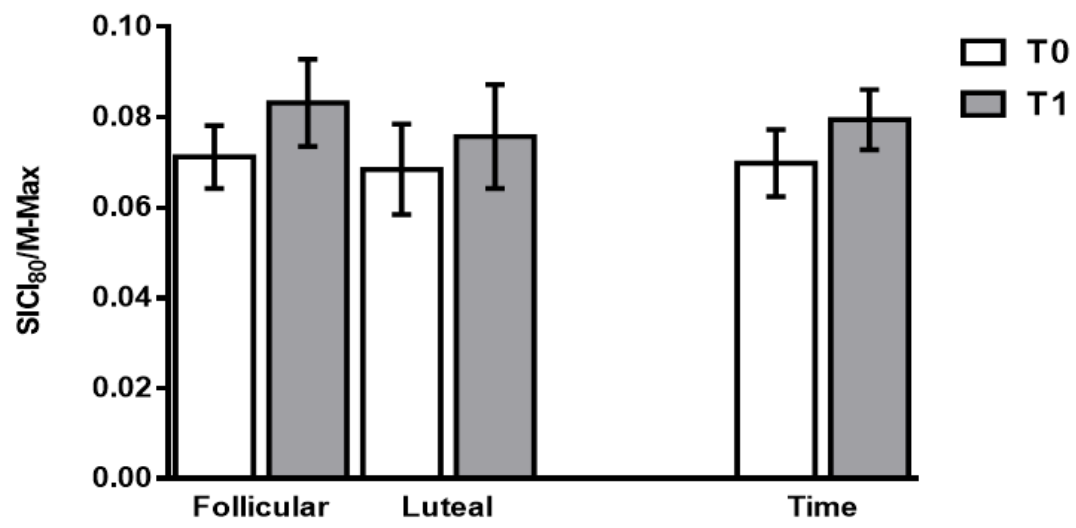

ii)

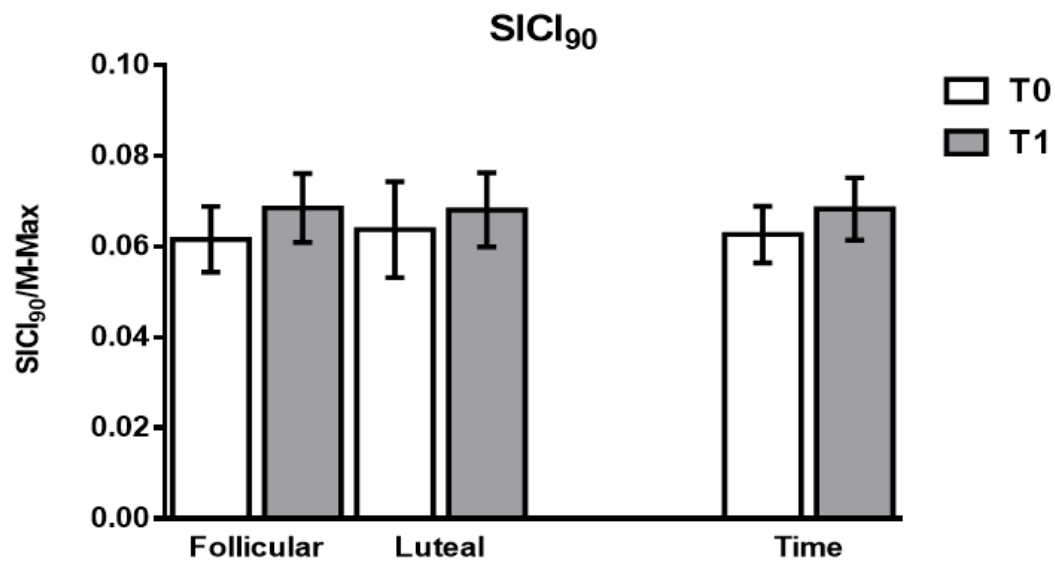

B)
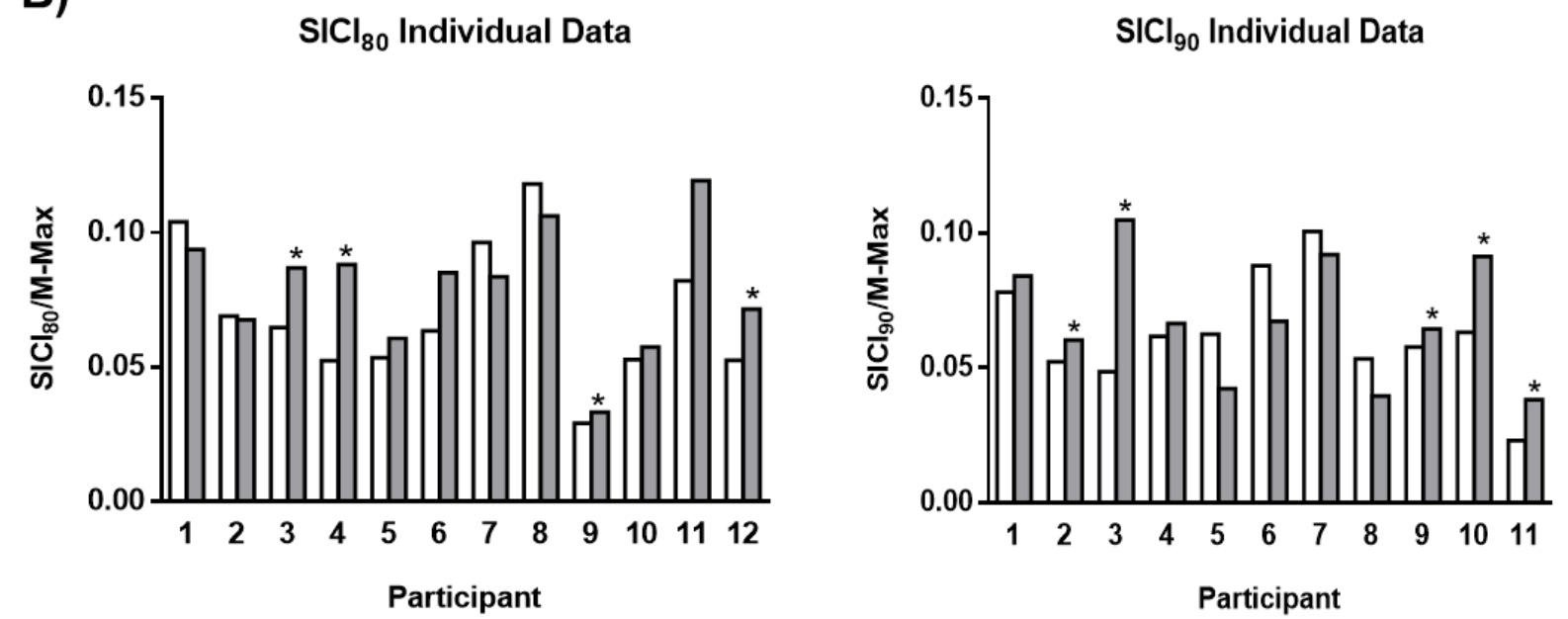


\section{M.Sc. Thesis - J. El-Sayes; McMaster University - Kinesiology}

Figure 5: SICI80 and SICI90 in Females. A) Two-way ANOVA in females revealed no main effects or interactions for: i) $\mathrm{SICI}_{80}$ or ii) $\mathrm{SICI}_{90}$ normalized to M-max. B) Individual data for $\mathrm{SICI}_{80}$ (left) and SICI 90 (right). SICI 80 was reduced in 7 (asterisks), increased in 3, and did not change in 2 females. SICI $_{90}$ was reduced in 5 (asterisks), increased in 3 , and did not change in 3 females. T0 (pre-exercise): white bars; T1 (post-exercise): gray bars. 
Figure 6

A) Males
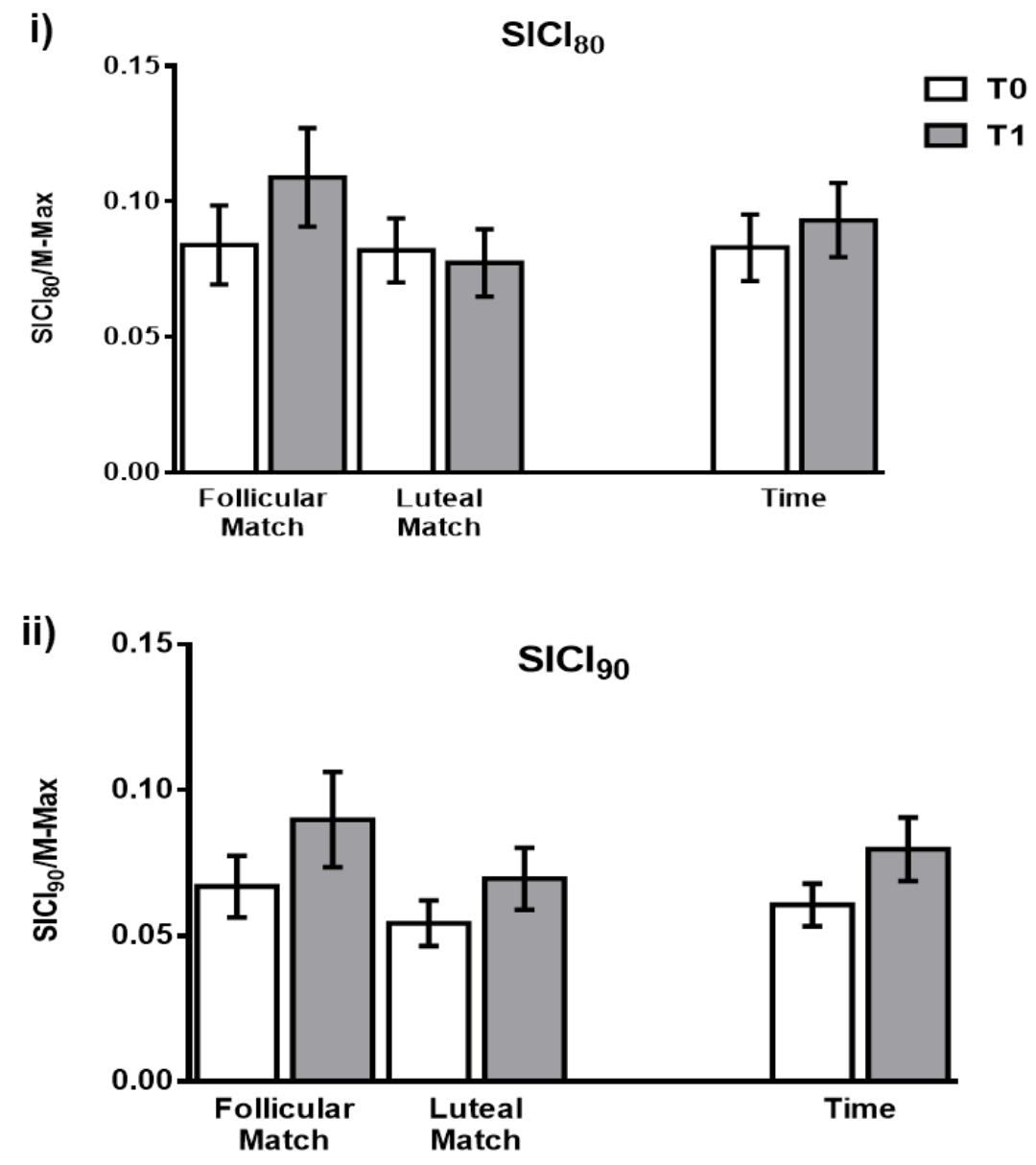

B)
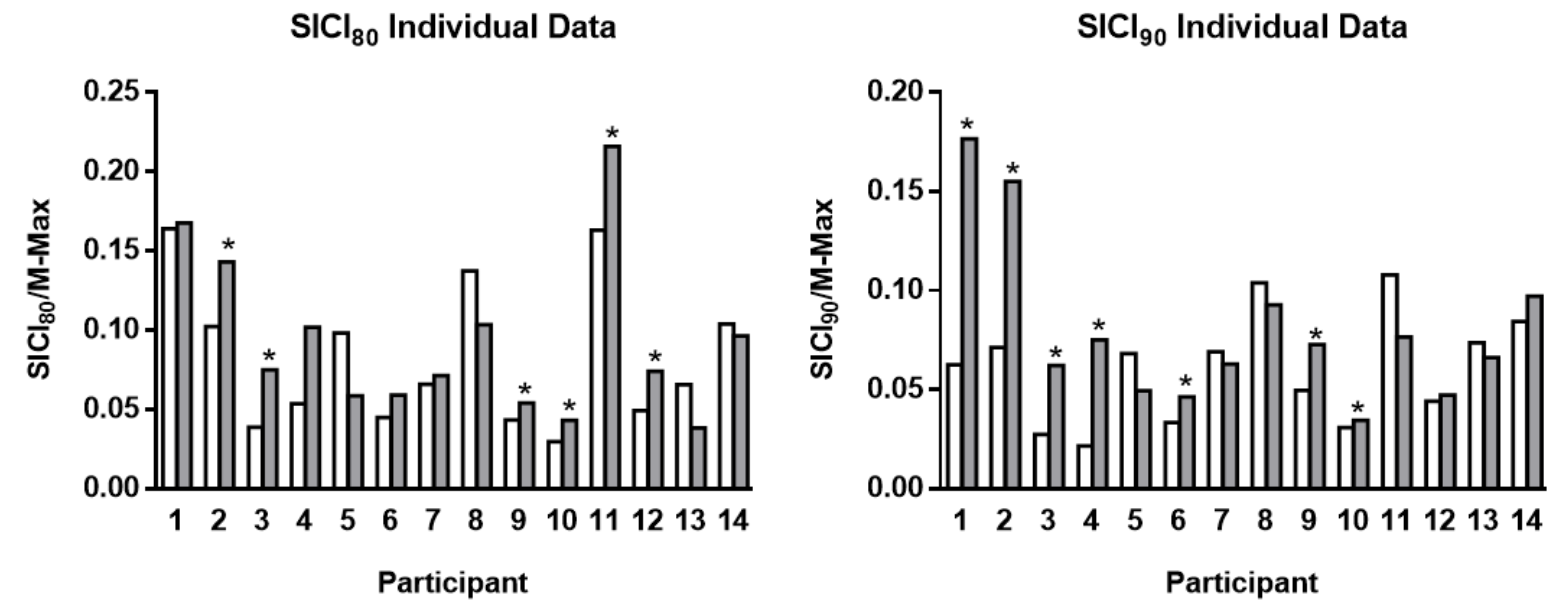
M.Sc. Thesis - J. El-Sayes; McMaster University - Kinesiology

Figure 6: SICI80 and SICI90 in Males: A) Two-way ANOVA in males revealed no main effects or interactions for: i) $\mathrm{SICI}_{80}$ or ii) $\mathrm{SICI}_{90}$ normalized to M-max. B) Individual data for $\mathrm{SICI}_{80}$ (left) and $\mathrm{SICI}_{90}$ (right). $\mathrm{SICI}_{80}$ was reduced in 8 (asterisks), increased in 3 , and did not change in 3 males. $\mathrm{SICI}_{90}$ was reduced in 8 (asterisks), increased in 4, and did not change in 2 males. T0 (preexercise): white bars; T1 (post-exercise): gray bars. 
M.Sc. Thesis - J. El-Sayes; McMaster University - Kinesiology

\section{Chapter 5: General Discussion and Conclusions}




\section{M.Sc. Thesis - J. El-Sayes; McMaster University - Kinesiology}

This thesis explored the effects of biological sex and ovarian hormones on neuroplasticity induced by a single session of aerobic exercise. The research assessed changes in excitation and inhibition in the primary motor cortex in males and females following acute aerobic exercise. Further, the effects of ovarian hormones were investigated by testing females at different points of the menstrual cycle. The findings from the research show that corticospinal excitability was enhanced following exercise in females but not males. This increase in corticospinal excitability was observed regardless of menstrual cycle phase. Further, the percent change in corticospinal excitability in females was associated with serum BDNF such that greater levels of BDNF were related to a greater percent change in corticospinal excitability. Last, there was a trend toward greater BDNF levels in females in the luteal phase compared to the follicular phase. Thus, this thesis revealed that biological sex, but not ovarian hormones, is an important factor that impacts the propensity for exercise-induced neuroplasticity.

\section{Limitations}

The findings presented here are limited to the effects of moderate intensity continuous exercise. Other acute exercise protocols may yield different findings. For example, high intensity exercise will likely reveal a different outcome, as it increases cortisol (Hill et al., 2008), which reduces excitability (Di et al., 2009; Sale et al., 2008), compared to the decrease in cortisol observed in this work. Therefore, high intensity exercise may not result in the increase in corticospinal excitability we observed in this work. Similarly, in this work, we did not observe an exercise-induced increase in corticospinal excitability in males. However, other exercise protocols may provide a more ideal stimulus to show this effect in males, and this would be an interesting and important follow-up to this thesis. 
The conclusions from this thesis are based on specific dependent measures acquired via TMS. We investigated corticospinal excitability through MEPs and $\mathrm{GABA}_{\mathrm{A}}-$ mediated cortical inhibition through SICI. We observed an effect of sex but not ovarian hormones on neuroplasticity induction. Specifically, only females showed an increase in corticospinal excitability following exercise, while males did not. It is important to note that a spinal component of corticospinal excitability (i.e. Hoffman reflex or H-reflex) was not assessed. Therefore, it is unclear if the changes in corticospinal excitability are mediated at the spinal or cortical level. Previous work has shown that the H-reflex decreases (Zehr et al., 2007) or does not change (Neva et al., 2017) following exercise, suggesting that the increases in corticospinal excitability observed in this work are cortically mediated. Further, although this work showed no effect of ovarian hormones on neuroplasticity induction, investigating other dependent measures may have revealed these effects. These dependent measures may include intracortical facilitation (ICF), which has been shown to increase (Singh et al., 2014) or decrease (Lulic et al., 2017) following exercise. This change may be impacted by the higher levels of progesterone in the luteal phase compared to the follicular phase. Progesterone is a positive modulator of $\mathrm{GABA}_{\mathrm{A}}$ receptors (Guennoun et al., 2015). Since ICF is reduced by $\mathrm{GABA}_{\mathrm{A}}$ receptor agonists (Ziemann et al., 2014), it is likely that higher levels of progesterone in the luteal phase impact the exercise-induced changes in ICF. As such, future studies should continue to examine the effects of biological sex and ovarian hormones on exerciseinduced neuroplasticity of other dependent measures.

Importantly, TMS literature is limited as it only provides an indirect assessment of neuroplasticity induction. Using TMS alone does not allow for the exploration of the mediators of the changes we observe (e.g. neurotransmitters). Therefore, future research would benefit from combining 
M.Sc. Thesis - J. El-Sayes; McMaster University - Kinesiology

different neuroscience techniques to assess changes we observe following exercise. For example, using a combination of TMS, magnetic resonance spectroscopy (MRS), and blood measures could reveal mediators of the increases in corticospinal excitability we observe. We observe an increase in corticospinal excitability, assessed via TMS. Does exercise also increase glutamate (via MRS) and peripheral BDNF (via blood) concentrations, and are these increases associated with the increase in MEP amplitude? As such, combining different neuroscience techniques allows for a more in depth investigation of exercise-induced neuroplasticity.

It has been previously stated that BDNF is an important mediator of exercise-induced neuroplasticity (Cotman et al., 2007). Our findings support this notion, as baseline peripheral BDNF levels were correlated with the percent change in corticospinal excitability following exercise. However, in this study, we did not assess BDNF levels post-exercise and cannot state that the increase we observed is due to an increase in BDNF post-exercise. Future work should investigate the relationship between changes in BDNF and changes in cortical excitability and inhibition following exercise. Although both peripheral BDNF and corticospinal excitability have been shown to increase following exercise, they have not been tested in combination. Increases in BDNF following exercise may allow for increases in corticospinal excitability through enhancing the amplitude and frequency of excitatory post-synaptic currents (Carmignoto et al., 1997; Lessmann et al., 1994; Levine et al., 1995). Therefore, it is likely that exercise will result in increases in BDNF and corticospinal excitability and that these increases will be correlated. While it has been suggested that peripheral BDNF levels may reflect brain BDNF levels (Krabbe et al., 2007), this remains unclear. BDNF is synthesized in both in the periphery (Wang et al., 2006) and in the central nervous system (Fujimura et al., 2002; Pan et al., 1998), but can only be obtained in 
vivo peripherally. BDNF is stored in platelets (Fujimura et al., 2002), and the analysis of BDNF levels is impacted by the completeness of blood clotting and the plasma isolation protocol (Fujimura et al., 2002). Further, ELISA kits used to analyze BDNF levels are susceptible to artifacts due to low specificity of BDNF antibodies and the presence of BDNF binding protein in the blood (Skornicka et al., 2002). Therefore, due to these confounding factors it remains unclear if obtained peripheral BDNF levels are a true reflection of brain BDNF.

The findings from this thesis are only generalizable to young, healthy, fit individuals with regular ovarian hormone fluctuations. It is unclear what outcomes we would observe in other populations. For example, how do oral contraceptives or menopause impact the results we observed? Due to lower levels of estrogen, postmenopausal women may not show increases in corticospinal excitability following exercise. Therefore, to apply the information present here to individuals outside our demographic, future research needs to investigate the effects of biological sex and ovarian hormones on exercise-induced neuroplasticity in other populations, such as elderly and clinical populations.

\section{Significance and Future Directions}

The findings presented in this thesis can guide future research investigating exercise-induced neuroplasticity. It is evident that males and females show different neurophysiological responses to acute exercise. However, it remains unclear what factors mediate these differences. Females have greater levels of estradiol, serotonin, dopamine, and BDNF compared to males (Laakso et al., 2002; Michalski et al., 2015; Ortiz et al., 1988; Weiss et al., 2005). We speculate that the 
M.Sc. Thesis - J. El-Sayes; McMaster University - Kinesiology

differential exercise-induced response we observe between sexes is driven by baseline differences of these neuromodulators. These neuromodulators increase excitability and therefore likely mediate the increased corticospinal excitability observed in females following exercise. It remains to be investigated if males and females show comparable increases in neuromodulators (e.g. serotonin, dopamine, and BDNF) following exercise. This will allow for a greater understanding of the differential response between males and females following acute aerobic exercise. Are the increases we observe in females but not males due to greater neuromodulator concentrations at baseline or due to a greater increase in these neuromodulators in females following exercise? Future research should investigate sex differences in exercise-induced changes of neuromodulators to further understand the differences in exercise-induced neuroplasticity between sexes.

Acute exercise has been used to promote motor learning (Skriver et al., 2014; Snow et al., 2016; Statton et al., 2015) and to promote neuroplasticity induction via non-invasive brain stimulation (NIBS) (Mang et al., 2016a, 2014; McDonnell et al., 2013). Future research will benefit from using the information provided in this thesis to improve their outcomes. Specifically, because acute exercise does not alter corticospinal excitability equally in males and females, they may not show similar benefits when combining exercise and motor learning or exercise and NIBS. Therefore, different protocols may be required to induce similar changes in males and females. Importantly, our findings also present important considerations that may impact the success of neuroplasticity inducing protocols in a rehabilitation setting. Therefore, biological sex should be considered when using exercise to promote neuroplasticity and functional recovery following neurological injury. 
M.Sc. Thesis - J. El-Sayes; McMaster University - Kinesiology

\section{Conclusion}

A single session of aerobic exercise is capable of inducing short-term neuroplasticity. Neuroplasticity induced by non-invasive brain stimulation has been shown to differ between sexes and across the menstrual cycle. The present thesis investigated whether biological sex and ovarian hormones also impact neuroplasticity induced by acute aerobic exercise. This thesis demonstrated that biological sex, but not ovarian hormones, impacts neuroplasticity induced by a single session of aerobic exercise. We observed an increase in corticospinal excitability in females only, and this did not differ across the menstrual cycle. Further, this increase in corticospinal excitability was positively associated with serum BDNF levels. This information can be used to guide future research and rehabilitation protocols aiming to induce neuroplasticity via acute exercise. 
M.Sc. Thesis - J. El-Sayes; McMaster University - Kinesiology

\section{References}

Abdoli-Eramaki, M., Damecour, C., Christenson, J., Stevenson, J., 2012. The effect of perspiration on the sEMG amplitude and power spectrum. J. Electromyogr. Kinesiol. 22, 908-913. https://doi.org/10.1016/j.jelekin.2012.04.009

Acheson, A., Conover, J.C., Fandl, J.P., DeChiara, T.M., Russell, M., Thadani, A., Squinto, S.P., Yancopoulos, G.D., Lindsay, R.M., 1995. A BDNF autocrine loop in adult sensory neurons prevents cell death. Nature 374, 450-453. https://doi.org/10.1038/374450a0

Altar, C.A., Boylan, C.B., Jackson, C., Hershenson, S., Miller, J., Wiegand, S.J., Lindsay, R.M., Hyman, C., 1992. Brain-derived neurotrophic factor augments rotational behavior and nigrostriatal dopamine turnover in vivo. Proc. Natl. Acad. Sci. U. S. A. 89, 11347-51.

Amin, Z., Mason, G.F., Cavus, I., Krystal, J.H., Rothman, D.L., Epperson, C.N., 2006. The interaction of neuroactive steroids and GABA in the development of neuropsychiatric disorders in women. Pharmacol. Biochem. Behav. 84, 635-643. https://doi.org/10.1016/j.pbb.2006.06.007

Banfi, G., Marinelli, M., Roi, G.S., Colombini, A., Pontillo, M., Giacometti, M., Wade, S., 1994. Growth hormone and insulin-like growth factor I in athletes performing a marathon at 4000 m of altitude. Growth Regul. 4, 82-6.

Barha, C.K., Davis, J.C., Falck, R.S., Nagamatsu, L.S., Liu-Ambrose, T., 2017a. Sex differences in exercise efficacy to improve cognition: A systematic review and meta-analysis of randomized controlled trials in older humans. Front. Neuroendocrinol. 46, 71-85. https://doi.org/10.1016/j.yfrne.2017.04.002

Barha, C.K., Falck, R.S., Davis, J.C., Nagamatsu, L.S., Liu-Ambrose, T., 2017b. Sex differences in aerobic exercise efficacy to improve cognition: A systematic review and meta-analysis of studies in older rodents. Front. Neuroendocrinol. 46, 86-105. https://doi.org/10.1016/j.yfrne.2017.06.001

Barha, C.K., Galea, L.A., Nagamatsu, L.S., Erickson, K.I., Liu-Ambrose, T., 2017c. Personalising exercise recommendations for brain health: Considerations and future directions. Br. J. Sports Med. 51, 636-639. https://doi.org/10.1136/bjsports-2016-096710

Barha, C.K., Hsiung, G.-Y.R., Best, J.R., Davis, J.C., Eng, J.J., Jacova, C., Lee, P.E., Munkacsy, M., Cheung, W., Liu-Ambrose, T., 2017d. Sex Difference in Aerobic Exercise Efficacy to Improve Cognition in Older Adults with Vascular Cognitive Impairment: Secondary Analysis of a Randomized Controlled Trial. J. Alzheimer's Dis. 60, 1397-1410. https://doi.org/10.3233/JAD-170221

Batra, N.A., Seres-Mailo, J., Hanstock, C., Seres, P., Khudabux, J., Bellavance, F., Baker, G., Allen, P., Tibbo, P., Hui, E., Le Melledo, J.M., 2008. Proton Magnetic Resonance Spectroscopy Measurement of Brain Glutamate Levels in Premenstrual Dysphoric Disorder. Biol. Psychiatry 63, 1178-1184. https://doi.org/10.1016/j.biopsych.2007.10.007

Batsikadze, G., Paulus, W., Kuo, M.-F., Nitsche, M.A., 2013. Effect of Serotonin on Paired Associative Stimulation-Induced Plasticity in the Human Motor Cortex.

Neuropsychopharmacology 38, 2260-2267. https://doi.org/10.1038/npp.2013.127 
Begliuomini, S., Casarosa, E., Pluchino, N., Lenzi, E., Centofanti, M., Freschi, L., Pieri, M., Genazzani, A.D., Luisi, S., Genazzani, A.R., 2007. Influence of endogenous and exogenous sex hormones on plasma brain-derived neurotrophic factor. Hum. Reprod. 22, 995-1002. https://doi.org/10.1093/humrep/del479

Benraiss, A., Chmielnicki, E., Lerner, K., Roh, D., Goldman, S.A., 2001. Adenoviral brainderived neurotrophic factor induces both neostriatal and olfactory neuronal recruitment from endogenous progenitor cells in the adult forebrain. J. Neurosci. 21, 6718-31.

Bestmann, S., Krakauer, J.W., 2015. The uses and interpretations of the motor-evoked potential for understanding behaviour. Exp. Brain Res. 233, 679-689. https://doi.org/10.1007/s00221-014-4183-7

Binder, D.K., Scharfman, H.E., 2004. Brain-derived neurotrophic factor. Growth Factors 22, 123-31.

Borg, G., 1990. Psychophysical scaling with applications in physical work and the perception of exertion. Scand. J. Work. Environ. Health 16 Suppl 1, 55-8.

Cappon, J., Brasel, J.A., Mohan, S., Cooper, D.M., 1994. Effect of brief exercise on circulating insulin-like growth factor I. J. Appl. Physiol. 76, 2490-6. https://doi.org/10.1152/jappl.1994.76.6.2490

Carbone, D.L., Handa, R.J., 2013. Sex and stress hormone influences on the expression and activity of brain-derived neurotrophic factor. Neuroscience 239, 295-303. https://doi.org/10.1016/j.neuroscience.2012.10.073

Carmignoto, G., Pizzorusso, T., Tia, S., Vicini, S., 1997. Brain-derived neurotrophic factor and nerve growth factor potentiate excitatory synaptic transmission in the rat visual cortex. $\mathrm{J}$. Physiol. 498 ( Pt 1), 153-64.

Carson, M.J., Behringer, R.R., Brinster, R.L., McMorris, F.A., 1993. Insulin-like growth factor I increases brain growth and central nervous system myelination in transgenic mice. Neuron $10,729-40$.

Chaieb, L., Antal, A., Paulus, W., 2008. Gender-specific modulation of short-term neuroplasticity in the visual cortex induced by transcranial direct current stimulation. Vis. Neurosci. 25, 77-81. https://doi.org/10.1017/S0952523808080097

Cheng, A., Coksaygan, T., Tang, H., Khatri, R., Balice-Gordon, R.J., Rao, M.S., Mattson, M.P., 2006. Truncated tyrosine kinase B brain-derived neurotrophic factor receptor directs cortical neural stem cells to a glial cell fate by a novel signaling mechanism. J. Neurochem. 0, 070209222715068-??? https://doi.org/10.1111/j.1471-4159.2006.04337.x

Cirillo, J., Lavender, A.P., Ridding, M.C., Semmler, J.G., 2009. Motor cortex plasticity induced by paired associative stimulation is enhanced in physically active individuals. J. Physiol. 587, 5831-5842. https://doi.org/10.1113/jphysiol.2009.181834

Coco, M., Perciavalle, V., Cavallari, P., Perciavalle, V., 2016. Effects of an Exhaustive Exercise on Motor Skill Learning and on the Excitability of Primary Motor Cortex and Supplementary Motor Area. Medicine (Baltimore). 95, e2978. 
M.Sc. Thesis - J. El-Sayes; McMaster University - Kinesiology

https://doi.org/10.1097/MD.0000000000002978

Cohen, J., 1988. Statistical Power Analysis for the Behavioral Sciences (2nd Edition) for the Behavioral Sciences (2nd Edition) ID : XM-77778.

Colcombe, S., Kramer, A.F., 2003. Fitness Effects on the Cognitive Function of Older Adults: A Meta-Analytic Study. Psychol. Sci. 14, 125. https://doi.org/10.1111/1467-9280.t01-1-01430

Conover, W.J., Iman, R.L., 1981. Rank Transformations as a Bridge between Parametric and Nonparametric Statistics. Am. Stat. 35, 124-129. https://doi.org/10.1080/00031305.1981.10479327

Cosgrove, K.P., Mazure, C.M., Staley, J.K., 2007. Evolving Knowledge of Sex Differences in Brain Structure, Function, and Chemistry. Biol. Psychiatry 62, 847-855. https://doi.org/10.1016/j.biopsych.2007.03.001

Cotman, C.W., Berchtold, N.C., 2002. Exercise: A behavioral intervention to enhance brain health and plasticity. Trends Neurosci. 25, 295-301. https://doi.org/10.1016/S01662236(02)02143-4

Cotman, C.W., Berchtold, N.C., Christie, L.A., 2007. Exercise builds brain health: key roles of growth factor cascades and inflammation. Trends Neurosci. 30, 464-472. https://doi.org/10.1016/j.tins.2007.06.011

Cubeddu, A., Bucci, F., Giannini, A., Russo, M., Daino, D., Russo, N., Merlini, S., Pluchino, N., Valentino, V., Casarosa, E., Luisi, S., Genazzani, A.R., 2011. Brain-derived neurotrophic factor plasma variation during the different phases of the menstrual cycle in women with premenstrual syndrome. Psychoneuroendocrinology 36, 523-530. https://doi.org/10.1016/j.psyneuen.2010.08.006

de Quervain, D.J.-F., Henke, K., Aerni, A., Treyer, V., McGaugh, J.L., Berthold, T., Nitsch, R.M., Buck, A., Roozendaal, B., Hock, C., 2003. Glucocorticoid-induced impairment of declarative memory retrieval is associated with reduced blood flow in the medial temporal lobe. Eur. J. Neurosci. 17, 1296-302.

Devanne, H., Lavoie, B.A., Capaday, C., 1997. Input-output properties and gain changes in the human corticospinal pathway. Exp. brain Res. 114, 329-38.

Di Lazzaro, V., Profice, P., Ranieri, F., Capone, F., Dileone, M., Oliviero, A., Pilato, F., 2012. Iwave origin and modulation. Brain Stimul. 5, 512-525. https://doi.org/10.1016/j.brs.2011.07.008

Di, S., Maxson, M.M., Franco, A., Tasker, J.G., 2009. Glucocorticoids Regulate Glutamate and GABA Synapse-Specific Retrograde Transmission via Divergent Nongenomic Signaling Pathways. J. Neurosci. 29, 393-401. https://doi.org/10.1523/JNEUROSCI.4546-08.2009

Ding, Y.-H., Li, J., Zhou, Y., Rafols, J.A., Clark, J.C., Ding, Y., 2006. Cerebral angiogenesis and expression of angiogenic factors in aging rats after exercise. Curr. Neurovasc. Res. 3, 1523.

El-Sayes, J., Harasym, D., Turco, C. V., Locke, M.B., Nelson, A.J., 2018. Exercise-Induced Neuroplasticity: A Mechanistic Model and Prospects for Promoting Plasticity. Neurosci. 
M.Sc. Thesis - J. El-Sayes; McMaster University - Kinesiology

107385841877153. https://doi.org/10.1177/1073858418771538

Elfving, B., Plougmann, P.H., Müller, H.K., Mathé, A.A., Rosenberg, R., Wegener, G., 2010. Inverse correlation of brain and blood BDNF levels in a genetic rat model of depression. Int. J. Neuropsychopharmacol. 13, 563-572. https://doi.org/10.1017/S1461145709990721

Fabel, K., Fabel, K., Tam, B., Kaufer, D., Baiker, A., Simmons, N., Kuo, C.J., Palmer, T.D., 2003. VEGF is necessary for exercise-induced adult hippocampal neurogenesis. Eur. J. Neurosci. 18, 2803-12.

Ferris, L.T., Williams, J.S., Shen, C.-L., 2007. The Effect of Acute Exercise on Serum BrainDerived Neurotrophic Factor Levels and Cognitive Function. Med. Sci. Sport. Exerc. 39, 728-734. https://doi.org/10.1249/mss.0b013e31802f04c7

Finocchi, C., Ferrari, M., 2011. Female reproductive steroids and neuronal excitability. Neurol. Sci. 32, 31-35. https://doi.org/10.1007/s10072-011-0532-5

Fujimura, H., Altar, C.A., Chen, R., Nakamura, T., Nakahashi, T., Kambayashi, J., Sun, B., Tandon, N.N., 2002. Brain-derived neurotrophic factor is stored in human platelets and released by agonist stimulation. Thromb. Haemost. 87, 728-34.

Garber, C.E., Blissmer, B., Deschenes, M.R., Franklin, B.A., Lamonte, M.J., Lee, I.M., Nieman, D.C., Swain, D.P., 2011. Quantity and quality of exercise for developing and maintaining cardiorespiratory, musculoskeletal, and neuromotor fitness in apparently healthy adults: Guidance for prescribing exercise. Med. Sci. Sports Exerc. 43, 1334-1359. https://doi.org/10.1249/MSS.0b013e318213fefb

Garnier, Y.M., Lepers, R., Stapley, P.J., Papaxanthis, C., Paizis, C., 2017. Changes in corticospinal excitability following uphill versus downhill treadmill exercise. Behav. Brain Res. 317, 242-250. https://doi.org/10.1016/j.bbr.2016.09.051

Gomez-Merino, D., Béquet, F., Berthelot, M., Chennaoui, M., Guezennec, C.Y., 2001. Sitedependent effects of an acute intensive exercise on extracellular 5-HT and 5-HIAA levels in rat brain. Neurosci. Lett. 301, 143-6.

Gorbet, D.J., Staines, W.R., 2011. Inhibition of contralateral premotor cortex delays visually guided reaching movements in men but not in women. Exp. Brain Res. 212, 315-325. https://doi.org/10.1007/s00221-011-2731-y

Grassi, S., Frondaroli, A., Scarduzio, M., Dutia, M.B., Dieni, C., Pettorossi, V.E., 2010. Effects of $17 \beta$-estradiol on glutamate synaptic transmission and neuronal excitability in the rat medial vestibular nuclei. Neuroscience $165,1100-1114$. https://doi.org/10.1016/j.neuroscience.2009.11.039

Griffin, É.W., Mullally, S., Foley, C., Warmington, S.A., O’Mara, S.M., Kelly, Á.M., Kelly, A.M., 2011. Aerobic exercise improves hippocampal function and increases BDNF in the serum of young adult males. Physiol. Behav. 104, 934-941.

https://doi.org/10.1016/j.physbeh.2011.06.005

Guennoun, R., Labombarda, F., Gonzalez Deniselle, M.C., Liere, P., De Nicola, A.F., Schumacher, M., 2015. Progesterone and allopregnanolone in the central nervous system: 
M.Sc. Thesis - J. El-Sayes; McMaster University - Kinesiology

Response to injury and implication for neuroprotection. J. Steroid Biochem. Mol. Biol. 146, 48-61. https://doi.org/10.1016/j.jsbmb.2014.09.001

Hallet, M., 2000. Transcranial Magnetic Stimulation and the Human Brain. Nature 406, 147150.

Hallett, M., 2007. Transcranial Magnetic Stimulation: A Primer. Neuron 55, 187-199. https://doi.org/10.1016/j.neuron.2007.06.026

Harel, N.Y., Martinez, S.A., Knezevic, S., Asselin, P.K., Spungen, A.M., 2015. Acute changes in soleus H-reflex facilitation and central motor conduction after targeted physical exercises. J. Electromyogr. Kinesiol. 25, 438-443. https://doi.org/10.1016/j.jelekin.2015.02.009

Heaney, J.L.J., Carroll, D., Phillips, A.C., 2013. DHEA, DHEA-S and cortisol responses to acute exercise in older adults in relation to exercise training status and sex. Age (Dordr). 35, 395405. https://doi.org/10.1007/s11357-011-9345-y

Hess, C.W., Mills, K.R., Murray, N.M., 1987. Responses in small hand muscles from magnetic stimulation of the human brain. J. Physiol. 388, 397-419.

Hill, E.E., Zack, E., Battaglini, C., Viru, M., Viru, A., Hackney, A.C., 2008. Exercise and circulating Cortisol levels: The intensity threshold effect. J. Endocrinol. Invest. 31, 587591. https://doi.org/10.1007/BF03345606

Homberg, J.R., Molteni, R., Calabrese, F., Riva, M.A., 2014. The serotonin-BDNF duo: Developmental implications for the vulnerability to psychopathology. Neurosci. Biobehav. Rev. 43, 35-47. https://doi.org/10.1016/j.neubiorev.2014.03.012

Hötting, K., Schickert, N., Kaiser, J., Röder, B., Schmidt-Kassow, M., 2016. The Effects of Acute Physical Exercise on Memory, Peripheral BDNF, and Cortisol in Young Adults. Neural Plast. 2016, 1-12. https://doi.org/10.1155/2016/6860573

Hsieh, J., Aimone, J.B., Kaspar, B.K., Kuwabara, T., Nakashima, K., Gage, F.H., 2004. IGF-I instructs multipotent adult neural progenitor cells to become oligodendrocytes. J. Cell Biol. 164, 111-122. https://doi.org/10.1083/jcb.200308101

Huang, T., Larsen, K.T., Ried-Larsen, M., Møller, N.C., Andersen, L.B.B., 2014. The effects of physical activity and exercise on brain-derived neurotrophic factor in healthy humans: A review. Scand. J. Med. Sci. Sport. 24, 1-10. https://doi.org/10.1111/sms.12069

Hübner, L., Voelcker-Rehage, C., 2017. Does physical activity benefit motor performance and learning of upper extremity tasks in older adults? - A systematic review. Eur. Rev. Aging Phys. Act. 14, 15. https://doi.org/10.1186/s11556-017-0181-7

Ida, M., Ida, I., Wada, N., Sohmiya, M., Tazawa, M., Shirakura, K., 2013. A clinical study of the efficacy of a single session of individual exercise for depressive patients, assessed by the change in saliva free cortisol level. Biopsychosoc. Med. 7, 18. https://doi.org/10.1186/17510759-7-18

Inghilleri, M., Conte, A., Currà, A., Frasca, V., Lorenzano, C., Berardelli, A., 2004. Ovarian hormones and cortical excitability. An rTMS study in humans. Clin. Neurophysiol. 115, 1063-1068. https://doi.org/10.1016/j.clinph.2003.12.003 
Jubeau, M., Rupp, T., Perrey, S., Temesi, J., Wuyam, B., Levy, P., Verges, S., Millet, G.Y., 2014. Changes in voluntary activation assessed by transcranial magnetic stimulation during prolonged cycling exercise. PLoS One 9. https://doi.org/10.1371/journal.pone.0089157

Kitaoka, R., Fujikawa, T., Miyaki, T., Matsumura, S., Fushiki, T., Inoue, K., 2010. Increased noradrenergic activity in the ventromedial hypothalamus during treadmill running in rats. J. Nutr. Sci. Vitaminol. (Tokyo). 56, 185-90.

Klein, A.B., Williamson, R., Santini, M.A., Clemmensen, C., Ettrup, A., Rios, M., Knudsen, G.M., Aznar, S., 2011. Blood BDNF concentrations reflect brain-tissue BDNF levels across species. Int. J. Neuropsychopharmacol. 14, 347-353. https://doi.org/10.1017/S1461145710000738

Koistinen, H., Koistinen, R., Selenius, L., Ylikorkala, Q., Seppala, M., 1996. Effect of marathon run on serum IGF-I and IGF-binding protein 1 and 3 levels. J. Appl. Physiol. 80, 760-764. https://doi.org/10.1152/jappl.1996.80.3.760

Kowiański, P., Lietzau, G., Czuba, E., Waśkow, M., Steliga, A., Moryś, J., 2017. BDNF: A Key Factor with Multipotent Impact on Brain Signaling and Synaptic Plasticity. Cell. Mol. Neurobiol. 38, 1-15. https://doi.org/10.1007/s10571-017-0510-4

Krabbe, K.S., Nielsen, A.R., Krogh-Madsen, R., Plomgaard, P., Rasmussen, P., Erikstrup, C., Fischer, C.P., Lindegaard, B., Petersen, A.M.W., Taudorf, S., Secher, N.H., Pilegaard, H., Bruunsgaard, H., Pedersen, B.K., 2007. Brain-derived neurotrophic factor (BDNF) and type 2 diabetes. Diabetologia 50, 431-438. https://doi.org/10.1007/s00125-006-0537-4

Kraemer, R.R., Durand, R.J., Acevedo, E.O., Johnson, L.G., Kraemer, G.R., Hebert, E.P., Castracane, V.D., 2004. Rigorous running increases growth hormone and insulin-like growth factor-I without altering ghrelin. Exp. Biol. Med. (Maywood). 229, 240-6.

Kujirai, T., Caramia, M.D., Rothwell, J.C., Day, B.L., Thompson, P.D., Ferbert, A., Wroe, S., Asselman, P., Marsden, C.D., 1993. Corticocortical inhibition in human motor cortex. J. Physiol. 471, 501-19.

Kumpulainen, S., Avela, J., Gruber, M., Bergmann, J., Voigt, M., Linnamo, V., MrachaczKersting, N., 2015. Differential modulation of motor cortex plasticity in skill- and endurance-trained athletes. Eur. J. Appl. Physiol. 115, 1107-1115. https://doi.org/10.1007/s00421-014-3092-6

Kuo, M.F., Paulus, W., Nitsche, M.A., 2006. Sex differences in cortical neuroplasticity in humans. Neuroreport 17, 1703-1707. https://doi.org/10.1097/01.wnr.0000239955.68319.c2

Laakso, A., Vilkman, H., Bergman, J., Haaparanta, M., Solin, O., Syvälahti, E., Salokangas, R.K.R., Hietala, J., 2002. Sex differences in striatal presynaptic dopamine synthesis capacity in healthy subjects. Biol. Psychiatry 52, 759-763. https://doi.org/10.1016/S00063223(02)01369-0

Lessmann, V., Gottmann, K., Heumann, R., 1994. BDNF and NT-4/5 enhance glutamatergic synaptic transmission in cultured hippocampal neurones. Neuroreport 6, 21-5.

Levine, E.S., Dreyfus, C.F., Black, I.B., Plummer, M.R., 1995. Brain-derived neurotrophic factor 
M.Sc. Thesis - J. El-Sayes; McMaster University - Kinesiology

rapidly enhances synaptic transmission in hippocampal neurons via postsynaptic tyrosine kinase receptors. Proc. Natl. Acad. Sci. U. S. A. 92, 8074-7.

Li, L., Men, W.-W., Chang, Y.-K., Fan, M.-X., Ji, L., Wei, G.-X., 2014. Acute Aerobic Exercise Increases Cortical Activity during Working Memory: A Functional MRI Study in Female College Students. PLoS One 9, e99222. https://doi.org/10.1371/journal.pone.0099222

Livingston, S.C., Goodkin, H.P., Ingersoll, C.D., 2010. The influence of gender, hand dominance, and upper extremity length on motor evoked potentials. J. Clin. Monit. Comput. 24, 427-36. https://doi.org/10.1007/s10877-010-9267-8

Lommatzsch, M., Zingler, D., Schuhbaeck, K., Schloetcke, K., Zingler, C., Schuff-Werner, P., Virchow, J.C., 2005. The impact of age, weight and gender on BDNF levels in human platelets and plasma. Neurobiol. Aging 26, 115-123. https://doi.org/10.1016/j.neurobiolaging.2004.03.002

Lopez-Lopez, C., LeRoith, D., Torres-Aleman, I., 2004. Insulin-like growth factor I is required for vessel remodeling in the adult brain. Proc. Natl. Acad. Sci. U. S. A. 101, 9833-8. https://doi.org/10.1073/pnas.0400337101

Louissaint, A., Rao, S., Leventhal, C., Goldman, S.A., 2002. Coordinated interaction of neurogenesis and angiogenesis in the adult songbird brain. Neuron 34, 945-60.

Lovallo, W.R., Robinson, J.L., Glahn, D.C., Fox, P.T., 2010. Acute effects of hydrocortisone on the human brain: an fMRI study. Psychoneuroendocrinology 35, 15-20. https://doi.org/10.1016/j.psyneuen.2009.09.010

Lulic, T., El-Sayes, J., Fassett, H.J., Nelson, A.J., 2017. Physical activity levels determine exerciseinduced changes in brain excitability. PLoS One 12, 1-18. https://doi.org/10.1371/journal.pone.0173672

MacIntosh, B.J., Crane, D.E., Sage, M.D., Rajab, A.S., Donahue, M.J., McIlroy, W.E., Middleton, L.E., 2014. Impact of a single bout of aerobic exercise on regional brain perfusion and activation responses in healthy young adults. PLoS One 9. https://doi.org/10.1371/journal.pone.0085163

Mang, C.S., Brown, K.E., Neva, J.L., Snow, N.J., Campbell, K.L., Boyd, L.A., 2016a. Promoting Motor Cortical Plasticity with Acute Aerobic Exercise: A Role for Cerebellar Circuits. Neural Plast. 2016. https://doi.org/10.1155/2016/6797928

Mang, C.S., Snow, N.J., Campbell, K.L., Ross, C.J.D., Boyd, L.A., 2014. A single bout of highintensity aerobic exercise facilitates response to paired associative stimulation and promotes sequence-specific implicit motor learning. J. Appl. Physiol. 117, 1325-1336. https://doi.org/10.1152/japplphysiol.00498.2014

Mang, C.S., Snow, N.J., Wadden, K.P., Campbell, K.L., Boyd, L.A., 2016b. High-Intensity Aerobic Exercise Enhances Motor Memory Retrieval. Med. Sci. Sport. Exerc. 48, 24772486. https://doi.org/10.1249/MSS.0000000000001040

Martinowich, K., Lu, B., 2008. Interaction between BDNF and Serotonin: Role in Mood Disorders. Neuropsychopharmacology 33, 73-83. https://doi.org/10.1038/sj.npp.1301571 
M.Sc. Thesis - J. El-Sayes; McMaster University - Kinesiology

Maruyama, A., Matsunaga, K., Tanaka, N., Rothwell, J.C., 2006. Muscle fatigue decreases shortinterval intracortical inhibition after exhaustive intermittent tasks. Clin. Neurophysiol. 117, 864-870. https://doi.org/10.1016/j.clinph.2005.12.019

McDonnell, M.N., Buckley, J.D., Opie, G.M., Ridding, M.C., Semmler, J.G., 2013. A single bout of aerobic exercise promotes motor cortical neuroplasticity. J. Appl. Physiol. 114, 1174-1182. https://doi.org/10.1152/japplphysiol.01378.2012

McGregor, K.M., Nocera, J.R., Sudhyadhom, A., Patten, C., Manini, T.M., Kleim, J.A., Crosson, B., Butler, A.J., 2013. Effects of aerobic fitness on aging-related changes of interhemispheric inhibition and motor performance. Front. Aging Neurosci. 5, 1-14. https://doi.org/10.3389/fnagi.2013.00066

Meeusen, R., Roeykens, J., Magnus, L., Keizer, H., De Meirleir, K., 1997. Endurance Performance in Humans: The Effect of a Dopamine Precursor or a Specific Serotonin (5-HT 2A/2C ) Antagonist. Int. J. Sports Med. 18, 571-577. https://doi.org/10.1055/s-2007-972683

Michalski, B., Corrada, M.M., Kawas, C.H., Fahnestock, M., 2015. Brain-derived neurotrophic factor and TrkB expression in the "oldest-old," the 90+ Study: correlation with cognitive status and levels of soluble amyloid-beta. Neurobiol. Aging 36, 3130-3139. https://doi.org/10.1016/j.neurobiolaging.2015.08.022

Möller, C., Arai, N., Lücke, J., Ziemann, U., 2009. Hysteresis effects on the input-output curve of motor evoked potentials. Clin. Neurophysiol. 120, 1003-8. https://doi.org/10.1016/j.clinph.2009.03.001

Monteggia, L.M., Barrot, M., Powell, C.M., Berton, O., Galanis, V., Gemelli, T., Meuth, S., Nagy, A., Greene, R.W., Nestler, E.J., 2004. Essential role of brain-derived neurotrophic factor in adult hippocampal function. Proc. Natl. Acad. Sci. U. S. A. 101, 10827-32. https://doi.org/10.1073/pnas.0402141101

Mooney, R.A., Coxon, J.P., Cirillo, J., Glenny, H., Gant, N., Byblow, W.D., 2016. Acute aerobic exercise modulates primary motor cortex inhibition. Exp. Brain Res. 234, 3669-3676. https://doi.org/10.1007/s00221-016-4767-5

Motl, R.W., Dishman, R.K., 2003. Acute leg-cycling exercise attenuates the H-reflex recorded in soleus but not flexor carpi radialis. Muscle and Nerve 28, 609-613. https://doi.org/10.1002/mus.10479

Murphy, D.D., Cole, N.B., Greenberger, V., Segal, M., 1998. Estradiol increases dendritic spine density by reducing GABA neurotransmission in hippocampal neurons. J. Neurosci. 18, 2550-9.

Nakamura, K., Tan, F., Li, Z., Thiele, C.J., 2011. NGF activation of TrkA induces vascular endothelial growth factor expression via induction of hypoxia-inducible factor-1 $\alpha$. Mol. Cell. Neurosci. 46, 498-506. https://doi.org/10.1016/j.mcn.2010.12.002

Nanda, B., Manjunatha, S., Balde, J., 2013. The Acute Effects of a Single Bout of Moderateintensity Aerobic Exercise on Cognitive Functions in Healthy Adult Males. J. Clin. Diagnositc Res. 7, 1883-5. https://doi.org/10.7860/JCDR/2013/5855.3341 
Neva, J.L., Brown, K.E., Mang, C.S., Francisco, B.A., Boyd, L.A., 2017. An acute bout of exercise modulates both intracortical and interhemispheric excitability. Eur. J. Neurosci. 45, 1343-1355. https://doi.org/10.1111/ejn.13569

Nieto-Estévez, V., Oueslati-Morales, C.O., Li, L., Pickel, J., Morales, A. V., Vicario-Abejón, C., 2016. Brain Insulin-Like Growth Factor-I Directs the Transition from Stem Cells to Mature Neurons During Postnatal/Adult Hippocampal Neurogenesis. Stem Cells 34, 2194-2209. https://doi.org/10.1002/stem.2397

Nofuji, Y., Suwa, M., Sasaki, H., Ichimiya, A., Nishichi, R., Kumagai, S., 2012. Different circulating brain-derived neurotrophic factor responses to acute exercise between physically active and sedentary subjects. J. Sports Sci. Med. 11, 83-8.

Ortiz, J., Artigas, F., Gelpí, E., 1988. Serotonergic status in human blood. Life Sci. 43, 983-990. https://doi.org/10.1016/0024-3205(88)90543-7

Ozyemisci-Taskiran, O., Gunendi, Z., Bolukbasi, N., Beyazova, M., 2008. The effect of a single session submaximal aerobic exercise on premotor fraction of reaction time: An electromyographic study. Clin. Biomech. 23, 231-235. https://doi.org/10.1016/j.clinbiomech.2007.08.027

Pan, W., Banks, W.A., Fasold, M.B., Bluth, J., Kastin, A.J., 1998. Transport of brain-derived neurotrophic factor across the blood-brain barrier. Neuropharmacology 37, 1553-61.

Park, J., Willoughby, D.S., Song, J.J., Leutholtz, B.C., Koh, Y., 2018. Exercise-induced changes in stress hormones and cell adhesion molecules in obese men. J. Inflamm. Res. 11, 69-75. https://doi.org/10.2147/JIR.S158294

Pencea, V., Bingaman, K.D., Wiegand, S.J., Luskin, M.B., 2001. Infusion of brain-derived neurotrophic factor into the lateral ventricle of the adult rat leads to new neurons in the parenchyma of the striatum, septum, thalamus, and hypothalamus. J. Neurosci. 21, 670617.

Pérez-Martín, M., Cifuentes, M., Grondona, J.M., López-Avalos, M.D., Gómez-Pinedo, U., García-Verdugo, J.M., Fernández-Llebrez, P., 2010. IGF-I stimulates neurogenesis in the hypothalamus of adult rats. Eur. J. Neurosci. 31, 1533-48. https://doi.org/10.1111/j.14609568.2010.07220.x

Piccinni, A., Marazziti, D., Del Debbio, A., Bianchi, C., Roncaglia, I., Mannari, C., Origlia, N., Catena Dell'Osso, M., Massimetti, G., Domenici, L., Dell'Osso, L., 2008. Diurnal variation of plasma brain-derived neurotrophic factor (BDNF) in humans: An analysis of sex differences. Chronobiol. Int. 25, 819-826. https://doi.org/10.1080/07420520802387773

Pitcher, J.B., Ogston, K.M., Miles, T.S., 2003. Age and sex differences in human motor cortex input-output characteristics. J. Physiol. 546, 605-13.

https://doi.org/10.1113/JPHYSIOL.2002.029454

Pluchino, N., Cubeddu, A., Begliuomini, S., Merlini, S., Giannini, A., Bucci, F., Casarosa, E., Luisi, M., Cela, V., Genazzani, A.R., 2009. Daily variation of brain-derived neurotrophic factor and cortisol in women with normal menstrual cycles, undergoing oral contraception and in postmenopause. Hum. Reprod. 24, 2303-2309. 
M.Sc. Thesis - J. El-Sayes; McMaster University - Kinesiology

https://doi.org/10.1093/humrep/dep119

Poo, M., 2001. Neurotrophins as synaptic modulators. Nat. Rev. Neurosci. 2, 24-32. https://doi.org/10.1038/35049004

Racinais, S., Girard, O., Micallef, J.P., Perrey, S., 2007. Failed Excitability of Spinal Motoneurons Induced by Prolonged Running Exercise. J. Neurophysiol. 97, 596-603. https://doi.org/10.1152/jn.00903.2006

Rajab, A.S., Crane, D.E., Middleton, L.E., Robertson, A.D., Hampson, M., MacIntosh, B.J., 2014. A single session of exercise increases connectivity in sensorimotor-related brain networks: a resting-state fMRI study in young healthy adults. Front. Hum. Neurosci. 8, 1-9. https://doi.org/10.3389/fnhum.2014.00625

Ridding, M.C., Ziemann, U., 2010. Determinants of the induction of cortical plasticity by noninvasive brain stimulation in healthy subjects. J. Physiol. 588, 2291-2304. https://doi.org/10.1113/jphysiol.2010.190314

Rioult-Pedotti, M.-S., Pekanovic, A., Atiemo, C.O., Marshall, J., Luft, A.R., 2015. Dopamine Promotes Motor Cortex Plasticity and Motor Skill Learning via PLC Activation. PLoS One 10, e0124986. https://doi.org/10.1371/journal.pone.0124986

Roig, M., Skriver, K., Lundbye-Jensen, J., Kiens, B., Nielsen, J.B., 2012. A Single Bout of Exercise Improves Motor Memory. PLoS One 7, 28-32. https://doi.org/10.1371/journal.pone.0044594

Rojas Vega, S., Hollmann, W., Vera Wahrmann, B., Strüder, H.K., 2012. pH buffering does not influence BDNF responses to exercise. Int. J. Sports Med. 33, 8-12. https://doi.org/10.1055/s-0031-1285929

Rossi, S., Hallett, M., Rossini, P.M., Pascual-Leone, A., Safety of TMS Consensus Group, 2009. Safety, ethical considerations, and application guidelines for the use of transcranial magnetic stimulation in clinical practice and research. Clin. Neurophysiol. 120, 2008-2039. https://doi.org/10.1016/j.clinph.2009.08.016

Sale, M. V., Ridding, M.C., Nordstrom, M.A., 2008. Cortisol Inhibits Neuroplasticity Induction in Human Motor Cortex. J. Neurosci. 28, 8285-8293. https://doi.org/10.1523/JNEUROSCI.1963-08.2008

Scharfman, H.E., MacLusky, N.J., 2006. Estrogen and brain-derived neurotrophic factor (BDNF) in hippocampus: complexity of steroid hormone-growth factor interactions in the adult CNS. Front. Neuroendocrinol. 27, 415-35. https://doi.org/10.1016/j.yfrne.2006.09.004

Scharfman, H.E., MacLusky, N.J., 2005. Similarities between actions of estrogen and BDNF in the hippocampus: coincidence or clue? Trends Neurosci. 28, 79-85. https://doi.org/10.1016/j.tins.2004.12.005

Schwarz, A.J., Brasel, J.A., Hintz, R.L., Mohan, S., Cooper, D.M., 1996. Acute effect of brief low- and high-intensity exercise on circulating insulin-like growth factor (IGF) I, II, and IGF-binding protein-3 and its proteolysis in young healthy men. J. Clin. Endocrinol. Metab. 81, 3492-3497. https://doi.org/10.1210/jcem.81.10.8855791 
Shibuya, K., Park, S.B., Geevasinga, N., Huynh, W., Simon, N.G., Menon, P., Howells, J., Vucic, S., Kiernan, M.C., 2016. Threshold tracking transcranial magnetic stimulation: Effects of age and gender on motor cortical function. Clin. Neurophysiol. 127, 2355-2361. https://doi.org/10.1016/j.clinph.2016.03.009

Sidhu, S.K., Cresswell, A.G., Carroll, T.J., 2012. Motor cortex excitability does not increase during sustained cycling exercise to volitional exhaustion. J. Appl. Physiol. 113, 401-409. https://doi.org/10.1152/japplphysiol.00486.2012

Siebner, H., Rothwell, J., 2003. Transcranial magnetic stimulation: new insights into representational cortical plasticity. Exp. Brain Res. 148, 1-16. https://doi.org/10.1007/s00221-002-1234-2

Siebner, H.R., Ziemann, U., 2014. What is the threshold for developing and applying optimized procedures to determine the corticomotor threshold? Clin. Neurophysiol. 125, 1-2. https://doi.org/10.1016/j.clinph.2013.07.012

Singh, A.M., Duncan, R.E., Neva, J.L., Staines, W.R., 2014. Aerobic exercise modulates intracortical inhibition and facilitation in a nonexercised upper limb muscle. BMC Sports Sci. Med. Rehabil. 6, 1-10. https://doi.org/10.1186/2052-1847-6-23

Singh, A.M., Staines, W.R., 2015. The effects of acute aerobic exercise on the primary motor cortex. J. Mot. Behav. 47, 328-339. https://doi.org/10.1080/00222895.2014.983450

Skornicka, E.L., Shi, X., Koo, P.H., 2002. Comparative binding of biotinylated neurotrophins to ?2-macroglobulin family of proteins: Relationship between cytokine-binding and neuromodulatory activities of the macroglobulins. J. Neurosci. Res. 67, 346-353. https://doi.org/10.1002/jnr.10097

Skriver, K., Roig, M., Lundbye-Jensen, J., Pingel, J., Helge, J.W., Kiens, B., Nielsen, J.B., 2014. Acute exercise improves motor memory: Exploring potential biomarkers. Neurobiol. Learn. Mem. 116, 46-58. https://doi.org/10.1016/j.nlm.2014.08.004

Smith, A.E., Goldsworthy, M.R., Garside, T., Wood, F.M., Ridding, M.C., 2014. The influence of a single bout of aerobic exercise on short-interval intracortical excitability. Exp. Brain Res. 232, 1875-1882. https://doi.org/10.1007/s00221-014-3879-Z

Smith, M.J., Adams, L.F., Schmidt, P.J., Rubinow, D.R., Wassermann, E.M., 2002. Effects of ovarian hormones on human cortical excitability. Ann. Neurol. 51, 599-603. https://doi.org/10.1002/ana.10180

Smith, M.J., Keel, J.C., Greenberg, B.D., Adams, L.F., Schmidt, P.J., Rubinow, D.A., Wassermann, E.M., 1999. Menstrual cycle effects on cortical excitability. Neurology 53, 2069-2069. https://doi.org/10.1212/WNL.53.9.2069

Snow, N.J., Mang, C.S., Roig, M., McDonnell, M.N., Campbell, K.L., Boyd, L.A., 2016. The Effect of an Acute Bout of Moderate-Intensity Aerobic Exercise on Motor Learning of a Continuous Tracking Task. PLoS One 11, e0150039. https://doi.org/10.1371/journal.pone.0150039

Sohrabji, F., Lewis, D.K., 2006. Estrogen-BDNF interactions: Implications for 
M.Sc. Thesis - J. El-Sayes; McMaster University - Kinesiology

neurodegenerative diseases. Front. Neuroendocrinol. 27, 404-414. https://doi.org/10.1016/j.yfrne.2006.09.003

Sonntag, W.E., Deak, F., Ashpole, N., Toth, P., Csiszar, A., Freeman, W., Ungvari, Z., 2013. Insulin-like growth factor-1 in CNS and cerebrovascular aging. Front. Aging Neurosci. 5, 27. https://doi.org/10.3389/fnagi.2013.00027

Statton, M.A., Encarnacion, M., Celnik, P., Bastian, A.J., 2015. A Single Bout of Moderate Aerobic Exercise Improves Motor Skill Acquisition. PLoS One 10, e0141393. https://doi.org/10.1371/journal.pone.0141393

Stavrinos, E.L., Coxon, J.P., 2017. High-intensity Interval Exercise Promotes Motor Cortex Disinhibition and Early Motor Skill Consolidation. J. Cogn. Neurosci. 29, 593-604. https://doi.org/10.1162/jocn_a_01078

Stillman, C.M., Cohen, J., Lehman, M.E., Erickson, K.I., 2016. Mediators of Physical Activity on Neurocognitive Function: A Review at Multiple Levels of Analysis. Front. Hum. Neurosci. 10, 626. https://doi.org/10.3389/fnhum.2016.00626

Strelzyk, F., Hermes, M., Naumann, E., Oitzl, M., Walter, C., Busch, H.-P., Richter, S., Schachinger, H., 2012. Tune It Down to Live It Up? Rapid, Nongenomic Effects of Cortisol on the Human Brain. J. Neurosci. 32, 616-625. https://doi.org/10.1523/JNEUROSCI.238411.2012

Studer, L., Spenger, C., Seiler, R.W., Othberg, A., Lindvall, O., Odin, P., 1996. Effects of brainderived neurotrophic factor on neuronal structure of dopaminergic neurons in dissociated cultures of human fetal mesencephalon. Exp. brain Res. 108, 328-36.

Sullivan, G.M., Feinn, R., 2012. Using Effect Size-or Why the P Value Is Not Enough. J. Grad. Med. Educ. 4, 279-82. https://doi.org/10.4300/JGME-D-12-00156.1

Tarnopolsky, M.A., 2008. Sex Differences in Exercise Metabolism and the Role of 17-Beta Estradiol. Med. Sci. Sport. Exerc. 40, 648-654. https://doi.org/10.1249/MSS.0b013e31816212ff

Thirugnanasambandam, N., Khera, R., Wang, H., Kukke, S.N., Hallett, M., 2015. Distinct interneuronal networks influence excitability of the surround during movement initiation. J. Neurophysiol. 114, 1102-8. https://doi.org/10.1152/jn.00791.2014

Thomas, R., Beck, M.M., Lind, R.R., Korsgaard Johnsen, L., Geertsen, S.S., Christiansen, L., Ritz, C., Roig, M., Lundbye-Jensen, J., 2016a. Acute Exercise and Motor Memory Consolidation: The Role of Exercise Timing. Neural Plast. 2016, 1-11. https://doi.org/10.1155/2016/6205452

Thomas, R., Flindtgaard, M., Skriver, K., Geertsen, S.S., Christiansen, L., Korsgaard Johnsen, L., Busk, D.V.P., Bojsen-Møller, E., Madsen, M.J., Ritz, C., Roig, M., Lundbye-Jensen, J., 2016. Acute exercise and motor memory consolidation: Does exercise type play a role? Scand. J. Med. Sci. Sports 27, 1523-1532. https://doi.org/10.1111/sms.12791

Thomas, R., Johnsen, L.K., Geertsen, S.S., Christiansen, L., Ritz, C., Roig, M., Lundbye-Jensen, J., 2016b. Acute Exercise and Motor Memory Consolidation: The Role of Exercise 
Intensity. PLoS One 11, e0159589. https://doi.org/10.1371/journal.pone.0159589

Torres-Aleman, I., Naftolin, F., Robbins, R.J., 1990. Trophic effects of insulin-like growth factor-I on fetal rat hypothalamic cells in culture. Neuroscience 35, 601-8.

Tranulis, C., Guéguen, B., Pham-Scottez, A., Vacheron, M.N., Cabelguen, G., Costantini, A., Valero, G., Galinovski, A., 2006. Motor threshold in transcranial magnetic stimulation: comparison of three estimation methods. Neurophysiol. Clin. Neurophysiol. 36, 1-7. https://doi.org/10.1016/j.neucli.2006.01.005

van Kuijk, A.A., Anker, L.C., Pasman, J.W., Hendriks, J.C.M., van Elswijk, G., Geurts, A.C.H., 2009. Stimulus-response characteristics of motor evoked potentials and silent periods in proximal and distal upper-extremity muscles. J. Electromyogr. Kinesiol. 19, 574-583. https://doi.org/10.1016/j.jelekin.2008.02.006

VanBruggen, M.D., Hackney, A.C., McMurray, R.G., Ondrak, K.S., 2011. The relationship between serum and salivary cortisol levels in response to different intensities of exercise. Int. J. Sports Physiol. Perform. 6, 396-407.

Vitrac, C., Benoit-Marand, M., 2017. Monoaminergic Modulation of Motor Cortex Function. Front. Neural Circuits 11, 72. https://doi.org/10.3389/fncir.2017.00072

Vitrac, C., Péron, S., Frappé, I., Fernagut, P.-O., Jaber, M., Gaillard, A., Benoit-Marand, M., 2014. Dopamine control of pyramidal neuron activity in the primary motor cortex via D2 receptors. Front. Neural Circuits 8, 13. https://doi.org/10.3389/fncir.2014.00013

Wang, H., Ward, N., Boswell, M., Katz, D.M., 2006. Secretion of brain-derived neurotrophic factor from brain microvascular endothelial cells. Eur. J. Neurosci. 23, 1665-1670. https://doi.org/10.1111/j.1460-9568.2006.04682.x

Weiss, L.A., Abney, M., Cook, E.H., Ober, C., 2005. Sex-Specific Genetic Architecture of Whole Blood Serotonin Levels. Am. J. Hum. Genet. 76, 33-41. https://doi.org/10.1086/426697

Weng, T.B., Pierce, G.L., Darling, W.G., Voss, M.W., 2015. Differential Effects of Acute Exercise on Distinct Aspects of Executive Function. Med. Sci. Sport. Exerc. 47, 1460 1469. https://doi.org/10.1249/MSS.0000000000000542

Wotton, C.A., Quon, E.F., Palmer, A.C., Bekar, L.K., 2018. Corticosterone and serotonin similarly influence GABAergic and purinergic pathways to affect cortical inhibitory networks. J. Neuroendocrinol. 30, e12592. https://doi.org/10.1111/jne.12592

Yamaguchi, T., Fujiwara, T., Liu, W., Liu, M., 2012. Effects of pedaling exercise on the intracortical inhibition of cortical leg area. Exp. Brain Res. 218, 401-406. https://doi.org/10.1007/s00221-012-3026-7

Yanagisawa, H., Dan, I., Tsuzuki, D., Kato, M., Okamoto, M., Kyutoku, Y., Soya, H., 2010. Acute moderate exercise elicits increased dorsolateral prefrontal activation and improves cognitive performance with Stroop test. Neuroimage 50, 1702-1710. https://doi.org/10.1016/j.neuroimage.2009.12.023

Zehr, E.P., Klimstra, M., Johnson, E.A., Carroll, T.J., 2007. Rhythmic leg cycling modulates 
forearm muscle H-reflex amplitude and corticospinal tract excitability. Neurosci. Lett. 419, 10-14. https://doi.org/10.1016/j.neulet.2007.03.045

Ziemann, U., Reis, J., Schwenkreis, P., Rosanova, M., Strafella, A., Badawy, R., MüllerDahlhaus, F., 2014. TMS and drugs revisited 2014. Clin. Neurophysiol. 126, 1847-68. https://doi.org/10.1016/j.clinph.2014.08.028

Zigova, T., Pencea, V., Wiegand, S.J., Luskin, M.B., 1998. Intraventricular administration of BDNF increases the number of newly generated neurons in the adult olfactory bulb. Mol. Cell. Neurosci. 11, 234-45. https://doi.org/10.1006/mcne.1998.0684

Zlotnik, A., Gruenbaum, B.F., Mohar, B., Kuts, R., Gruenbaum, S.E., Ohayon, S., Boyko, M., Klin, Y., Sheiner, E., Shaked, G., Shapira, Y., Teichberg, V.I., 2011. The Effects of Estrogen and Progesterone on Blood Glutamate Levels: Evidence from Changes of Blood Glutamate Levels During the Menstrual Cycle in Women. Biol. Reprod. 84, 581-586. https://doi.org/10.1095/biolreprod.110.088120

Zoghi, M., Vaseghi, B., Bastani, A., Jaberzadeh, S., Galea, M.P., 2015. The effects of sex hormonal fluctuations during menstrual cycle on cortical excitability and manual dexterity (a pilot study). PLoS One 10,1-14. https://doi.org/10.1371/journal.pone.0136081 